Nulsen, P. E. J. (1982), MNRAS, 199, 1089

Pagel, B. E. J Patchett, B. E. (1975), MNRAS, 172, 13

Peoble P. J. E Nuseer, A (2010), Nature 465, 565

Peebic, A. O., Rupen, M. P. (2007), AJ, 134, 1052

Platen, E. (2010), PhD thesis, University of Groningen

Poggianti, B. M. Smail, I. Dressler, A. et al. (1999); ApJ, 518, 576

Postman, M., Geller, M. J. (1984), ApJ, 281, 95

Pustilnik, S. A., Martin, J.-M., Tepliakova, A. L., Kniazev, A. Y. (2011), MNRAS, 417,1335

Putman, M. E., Peek, J. E. G., Joung, M. R. (2012), ARA\&SA, 50, 491

Richter, O-G., Sancisi, R. (1994), A\& A, 290, 9

Roediger, E. (2009), AN, 330, 888

Roediger, E. (2009), An, (2008), A\&SARv, 15, 189

Sancisi, R., Thatenali, T., Oosterloo, T., van der Hulst, T. (2012), AJ in press

Schiminovich, D., van Gorkom, J. H., van der Hulst, J. M. (2012), 18 , 1 ., Oosterloo, T., Morganti, R. et al. (2012), MNRAS, 422, 1835

Shibata, R., Matsushita, K., Yamasaki, N. Y. et al. (2001), ApJ, 549, 228

Solanes, J., Manrique, A., García-Gómez, C. et al. (2001), ApJ, 548, 97

Stanonik, K., Platen, E., Aragón-Calvo, M. A. et al. (2009), ApJL, 696, 6

Tonnesen, S., Bryan, G. L. (2008), ApJL, 684, 9

Tonnesen, S., Bryan, G. L. (2009), ApJ, 694, 789

Tonnesen, S., Bryan, G. L. (2010), ApJ, 709, 1203

Tonnesen, S., Bryan, G. L. (2011), ApJ, 731, 98

Tonnesen, S., Bryan. G. L., van Gorkom, J. H. (2007), ApJ, 671, 1434

Tonn

Martinez, Saar, Gonzales, Pons-Borderia, eds. , Berlin: Springer, 291

Martinez, Saar, Gonzales, Pons-Borderia, eds., B
der Hulst, J. M., Sancisi, R. (1988), AJ, 95, 1354

van Gorkom, J. H. (2004), in Clusters of Galaxies: Probes of Cosmologica Structure and Galaxy Evolution, J. S. Mulchaey, A. Dressler \& Oemler, A. eds., Cambridge: Cambridge University Press, p. 304

Vollmer, B. (2009), A\&A, 502, 427

Vollmer, B. Beck, R., Kenney, J. P. D., van Gorkom, J. H. (2004), AJ, 127, 3375

Vollmer, B Cayatte, V. Balkowski, C.. Duschl, W. J. (2001), ApJ, 561, 708

Volmer, B. Marcelin, M Amran, P. et al. (2000), A\&A, 364, 532

Vollmer, B., Soida, M. Otmianowrska-Mazur, K. et al. (2006), A\&A, 453, 883

\section{Evolution of star formation and gas}

Nick Z. Scoville

California Institute of Technology

1200 East California Boulevard, Pasadena, CA 91125, USA

NZS@astro.caltech.edu

\section{Abstract}

In these lectures I review observations of star-forming molecular clouds in our Galaxy and nearby galaxies to develop a physical intuition for understanding star formation in the local and high-redshift Universe. A lot of this material is drawn from early work in the field since much of the work was done two decades ago and this background is not generally available in the present literature. I also attempt to synthesise our well-developed understanding of star formation in low-redshift galaxies with constraints from theory and observations at high redshift to develop an intuitive model for the evolution of galaxy mass and luminosity functions in the early Universe.

The overall goal of this contribution is to provide students with background helpful for analysis of far-infrared (FIR) observations from Herschel and millimetre/submillimetre $(\mathrm{mm} / \mathrm{submm})$ imaging with ALMA (the Atacama Large Millimetre/submillimetre Array). These two instruments will revolutionise our understanding of the interstellar medium (ISM) and associated star formation and galaxy evolution, both locally and in the distant Universe. To facilitate interpreting the FIR spectra of Galactic star-forming regions and high-redshift sources, I develop a model for the dust heating and radiative transfer in order to elucidate the observed infrared (IR) emissions. I do this because I am not aware of a similar coherent discussion in the literature.

\subsection{Star-forming molecular clouds}

Here, I provide an overview of the observations and physics of star-forming molecular clouds in the Galaxy and nearby normal galaxies. The motivation is to develop the intuitive background for analysis of observations at high redshift, hopefully avoiding naive and simplistic assumptions. The physics 
of the molecular ISM is not at all what one would have guessed before the advent of mm-line astronomy and the understanding developed over the last 30 years is vital to galactic evolution studies.

\subsubsection{Background}

All known star formation in low-redshift galaxies occurs in molecular gas clouds and inclusion of atomic Hr clouds in discussions of star formation is a 'red herring', except in as much as the atomic gas may feed the buildup of molecular clouds. In fact, there is no instance of star formation demonstrably observed to occur in atomic gas in nearby galaxies (as distinct from atomic gas dissociated by recently formed stars). Even in the extended ultraviolet (UV) disks of nearby galaxies, the young stars producing the UV could have been formed from molecular clouds simply too low in mass or surface density to be detected in present observations. The dominant process for formation of hydrogen molecules $\left(\mathrm{H}_{2}\right)$ at low redshift is believed to be on the surface of dust grains where two $\mathrm{H}$ atoms remain in proximity long enough for a radiative decay to a bound state. The hydrogen molecule is dissociated from the ground state by a two-step process involving UV photon absorption in the Lyman and Werner bands longward of the HI Lyman limit, followed by radiative decay to unbound vibrational states in the ground electronic state (Hollenbach et al. 1971). Thus, the destruction rate for $\mathrm{H}_{2}$ depends sensitively on the ambient UV radiation field and hence on the amount of dust extinction and $\mathrm{H}_{2}$ column density to the cloud surface - the latter since the foreground $\mathrm{H}_{2}$ may absorb all the incoming UV when the $\mathrm{H}_{2}$ Lyman and Werner bands become optically thick (i.e., self-shielding). Thus, whether a gas parcel is atomic or molecular will depend complexly on a number of factors: the volume density (since the formation rate depends on the densities of dust and atoms), the clumping of the ISM and the column density to the cloud surface and velocity dispersion which determine the dust continuum and $\mathrm{H}_{2}$ line opacities. For typical cloud densities of a few hundred per $\mathrm{cm}^{-3}$, the phase transition from $\mathrm{HI}$ - to $\mathrm{H}_{2}$-dominated will occur at a column density $N_{\mathrm{H}+2 \mathrm{H}_{2}} \sim 2 \times 10^{21} \mathrm{~cm}^{-2}$, corresponding to an extinction $A_{V}=1 \mathrm{mag}$ for a standard dust to gas abundance ratio (Hollenbach et al. 1971).

The $\mathrm{H}_{2}$ molecule is homonuclear and therefore has no permanent dipole moment - its pure rotational transitions are weak quadrupole transitions with low emissivity. In addition, $\mathrm{H}_{2}$ has a small moment of inertia and its first rotational transition $(J=2-0)$ at $28.2 \mu \mathrm{m}$ is therefore at relatively high energy $(h \nu / k \simeq 508 \mathrm{~K})$, requiring a minimum gas temperature of $T_{\mathrm{k}} \sim 150 \mathrm{~K}$ for appreciable collisional excitation. Thus trace molecules such as $\mathrm{CO}$ and

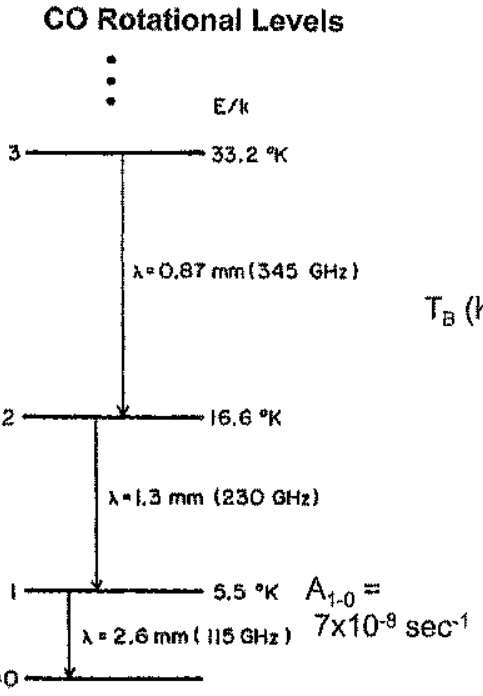

$\operatorname{CO}(\mathrm{J}=1-0)$ in Orion KL. Nebula
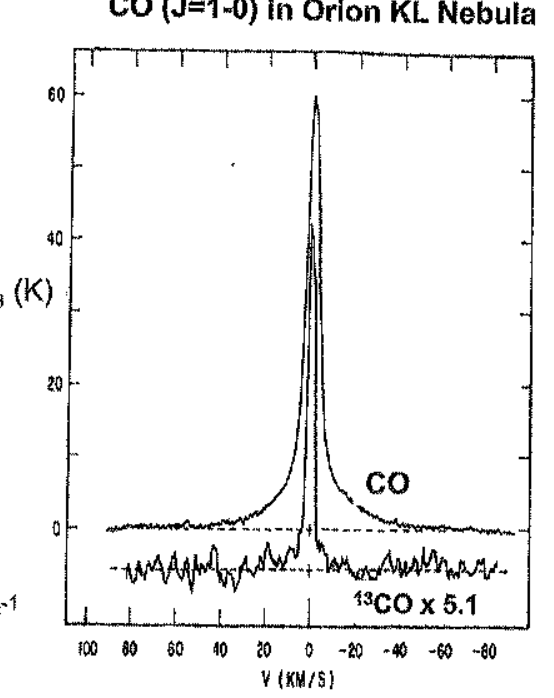

Fig. 8.1. The low-lying rotational levels of $\mathrm{CO}$ are shown on the left; the spontaneous decay rate for the $J=1-0$ transition is $7 \times 10^{-8} \mathrm{sec}^{-1}$ and the critical density for pure collisional excitation by $\mathrm{H}_{2}$ is $\sim 3000 \mathrm{~cm}^{-3}$. With line photor trapping and a typical optical depth of $\tau \sim 10$, the critical density for thermalisation is reduced to $\sim 300 \mathrm{~cm}^{-3}$. Thus, the $\mathrm{CO}$ line brightness temperature provides a measure of the gas kinetic temperature. On the right, the $\mathrm{CO}$ and ${ }^{13} \mathrm{CO} J=1-0$ emission lines are shown in the direction of the Orion Kleinmann-Low (KL) nebula.

HCN have become the standard surrogate probes of molecular clouds. CO and HCN have dipole moments of 0.1 and 2.3 Debye, respectively, and their larger moments of inertia (due to having heavier atoms than $\mathrm{H}$ ) yield lowest rotational transitions at 115.2 and $88.6 \mathrm{GHz}$ ( 2.6 and $3 \mathrm{~mm}$ wavelength), corresponding to $h \nu / k \simeq 5.5$ and $4.2 \mathrm{~K}$ (see Fig. 8.1). These molecules also have rare isotopic forms (e.g., ${ }^{13} \mathrm{CO}$ and $\mathrm{C}^{18} \mathrm{O}$ ) with slightly different moments of inertia, and hence shifted rotational lines which can be used to probe regions of high column density where the abundant isotope lines are very optically thick.

\subsubsection{Molecular excitation}

Excitation for the rotational levels of molecules like CO observed at $\mathrm{mm}$ and submm wavelengths is provided by collisions with $\mathrm{H}_{2}$. If these collisions are sufficiently frequent (compared to the spontaneous decay rate) the levels will come into thermal equilibrium with the $\mathrm{H}_{2}$ and their excitation temperature will approach the gas kinetic temperature. For optically 
thin transitions, this occurs at the critical density $n_{\mathrm{H}_{2}, \text { crit }}=A_{\mathrm{u}-1} /\left\langle\sigma \nu_{\mathrm{u}-1}\right\rangle$ where $A_{\mathrm{u}-1}$ is the Einstein spontaneous decay rate $\left(7 \times 10^{-8} \mathrm{sec}^{-1}\right.$ for $\mathrm{CO}$ $=1-0)$ and $\left\langle\sigma \nu_{u-1}\right\rangle$ is the collisional de-excitation rate averaged over the velocities. For CO $(J=1-0)$ thi critical density is $\sim 3000 \mathrm{~cm}^{-3}$ and for $\mathrm{HCN}$ (and most other molecules) it is $\sim 10^{4-5} \mathrm{~cm}^{-3}$ due to their higher dipole moments and therefore higher $A$ con molecules are generally taken as probes of high-density molecular gas as compared with that traced by $\mathrm{CO}$.

列 ${ }^{13} \mathrm{C}$ isotopes of both $\mathrm{CO}$ and $\mathrm{HCN}$ indicate that the ${ }^{12} \mathrm{CO}$ emission is optically thick. The rare isotopes are typically een at an intensity approximately $0.1-0.5$ of the abundant species whereas the ISM abundance ratio is ${ }^{13} \mathrm{C} / \mathrm{C}=1 / 89-1 / 40$, implying that the emission from the abundant species is optically thick and therefore saturated (see Fig. 81, right). For optically thick transitions, the upper-level population ig. 8.1, right). For optically thick transitions, the upper-level population can be enhanced due to absorption of line photons, leading to excitation temperatures higher than those expected simply due to $\mathrm{H}_{2}$ collisions, since the line photons emitted upon spontaneous decay cannot easily escape the cloud. This so-called radiative trapping of the line photons builds up the radiation field at the frequency of the line, leading to enhanced excitation of the upper state via photon absorption.

The escape probability formalism used to treat this optically thick situaThe escape solomon (1974) and Goldreich \& Kwan (1974) and is now routinely used to analyse interstellar molecule excitation (often called large velocity gradient [LVG], non-thermal molysis; see, e.g., van der Tak et al. (2007) and their equilibrium [non-LTE] analysis; see, e.g., van der Tak et al. (2007) and their publicly available RADEX code ${ }^{\dagger}$. One of the biggest advantages of the LVG formalism is that it permits treatment of the coupled radiative transfer and molecular excitation as a local problem - a fact probably not fully appreciated by current routine users of these codes. This formalism is applicable to ituations in which systematic velocity gradients are large compared to the 列 line photons from one region of the cloud small-scale thermal motions. The line photons the the Dopler shift they then only interact with molecules in the local region near where they were emitted.

In the photon trapping regime, the spontaneous decay rates $(A)$ used in analysing the equilibrium molecular excitation are reduced by a factor $\beta$ equal to the effective probability for escape of line photons from the emisequal to the (Scoville \& Solomon 1974; Goldreich \& Kwan 1974). Thus, the

† http://www.strw.leidenuniv,nl/ moldata/radex.htmI critical density for thermalisation of the levels is reduced by the same factor $\beta$. For a spherically symmetric cloud with radial velocity field $(\nu \propto r)$, the escape probability is given by

$$
\beta=\frac{1}{\tau}\left(1-\mathrm{e}^{-\tau}\right)
$$

where

$$
\tau=\frac{A_{\mathbf{u}-1} \lambda^{3} g_{\mathrm{u}}}{8 \pi \mathrm{d} \nu / \mathrm{d} r}\left(n_{\mathrm{l}} / g_{1}-n_{\mathrm{u}} / g_{\mathrm{u}}\right)
$$

for $\tau \ll 1, \beta=1$ and for $\tau \gg 1, \beta=1 / \tau$. Thus the critical density is reduced by a factor of $\tau$ in the optically thick regime. For most giant molecular clouds (GMCs - see below), $\tau_{\mathrm{CO}} \gtrsim 10$ (based on the relative strength of ${ }^{13} \mathrm{CO}$ ), so the critical density of $\mathrm{CO}(1-0)$ is reduced from 3000 to $300 \mathrm{~cm}^{-3}$. Thus, the CO excitation temperature will be approximately thermalised at the gas kinetic temperature $\left(T_{\mathrm{k}}\right)$ in most clouds. And since the $C O$ line is optically thick, the brightness temperature at the emission line peak will indicate the $\mathrm{H}_{2}$ kinetic temperature.

Similar considerations apply for the optically thick lines of molecules such as HCN and CS which have higher dipole moments - i.e., their critical densities are reduced by a factor $1 / \tau$ but since those critical densities are higher than that of $\mathrm{CO}$, their excitation temperatures and line brightness temperatures will be subthermal (except in the densest cloud core regions). In fact, since the critical density for optically thick transitions is $n_{\mathrm{H}_{2}, \text { crit }}=\left(A_{\mathfrak{u}-1} / \tau\right) /\left\langle\sigma \nu_{\mathbf{u}-1}\right\rangle$, the radiative line strength (appearing in the $A$ coefficient in the numerator and in $\tau$ in the denominator) cancels out - i.e. the critical density then just depends inversely on the molecular abundance.

In the regime of photon trapping, one should not assume that the emission of a rare isotope like ${ }^{13} \mathrm{CO}$ is optically thin simply because it is observed to have lower intensity than the abundant isotope emission. The rare isotope may simply have a lower excitation temperature since it has less photon trapping. This fact is under-appreciated by 'casual' observers. Typically, the ${ }^{13} \mathrm{CO}$ emission is $1 / 5$ to $1 / 10$ of $\mathrm{CO}$ - does this mean the optical depth is always in the small range $1 / 5$ to $1 / 10$ ? - of course not!

Molecular excitation can also be provided by absorption of continuum photons - either the IR continuum of dust or the cosmic microwave background radiation (CMB). In the escape probability formalism it is easy to include such continuum excitation. If $\beta$ is the probability of a line photon escaping from the local region, then $\beta$ is also the probability of an externally produced background photon making it into the local volume. The low-lying molecular levels will maintain a base excitation temperature at the cosmic 
background level, $2.7 \times(1+$ redshift $) \mathrm{K}$ (even in the absence of $\mathrm{H}_{2}$ collisions). However, since the line observations measure only the excess emission above the local continuum background, this CMB excitation does not result in detectable emission. The continuum from dust emission is not generally important for exciting low-lying molecular levels at $\mathrm{mm}$ wavelengths (since the continuum is optically thin and weak); but for higher-energy transitions at $\lambda_{\text {rest }}<500 \mu \mathrm{m}$ the IR continuum should be included.

\subsubsection{Summary}

In summary, we can expect that:

(a) The low $\mathrm{CO}$ levels will be thermalised and the brightness temperatures (a observed in spatiajly resolved clouds) will approximate the gas kinetic temperature, i.e., $T_{\mathrm{B}}(\mathrm{CO}) \simeq T_{\mathrm{k}}\left(\mathrm{H}_{2}\right)$.

(b) Photon trapping in optically thick lines can greatly enhance the molecular excitation above the excitation due to collisions with $\mathrm{H}_{2}$ molecules.

(c) The $\mathrm{CO}$ emission will trace the overall distribution of $\mathrm{H}_{2}$ for densities $n_{\mathrm{H}_{2}} \sim$ $10^{2-3} \mathrm{~cm}^{-3}$ - this turns out to be well tuned to characteristic densities of the majority of the molecular gas mass.

(d) The emissions from higher dipole moment molecules (HCN, CS, etc.) will trace higher density regions with $n_{\mathrm{H}_{2}} \geq 10^{4-5} \mathrm{~cm}^{-3}$.

\subsubsection{Observed properties of molecular gas}

Extensive surveys of the $\mathrm{CO}$ and ${ }^{13} \mathrm{CO}(J=1-0$ at $\lambda=2.6 \mathrm{~mm}$ and $J=2-1$ at $\lambda=1.3 \mathrm{~mm}$ ) emissions in the Galaxy have been used to determine the overall distribution of star-forming $\mathrm{H}_{2}$ gas and its properties (Scoville \& Solomon 1975; Sanders et al. 1985; Dame \& Thaddeus 1985; Scoville et al. 1987; Clemens et al. 1988; Matsunaga et al. 2001; Dame et al. 2001; Jackson et al. 2006). CO emission is ubiquitous at all Galactic longitudes in the inner Galaxy $\left(|l|<90^{\circ}\right)$ and within $1^{\circ}$ latitude of the Galactic plane. The $\mathrm{CO}$ emission is particularly strong in the central $1^{\circ}$ around the Galactic centre and within an annulus/ring from $|l|=20-50$ degrees - the so-called molecular cloud ring at $3-7 \mathrm{kpc}$ radius. This concentration of the $\mathrm{H}_{2}$ to the interior of the Galaxy is remarkably different from that of $\mathrm{HI}$; the 21-cm HI distribution is much smoother and the overall $\mathrm{HI}$ distribution is fairly constant with Galactic radius out to $R \sim 12-15 \mathrm{kpc}$ with mean column density perpendicular to the plane of $\left\langle N_{\mathrm{H}}\right\rangle \simeq 10^{21} \mathrm{~cm}^{-2}$ (see Fig. 8.2).

Within the Galaxy the overall mass contents of the molecular and atomic gas are approximately equal $\left(\sim 2 \times 10^{9} M_{\odot}\right.$ in each) but most of the molecular gas is inside the Solar-circle whereas most of the HI is outside $8.5 \mathrm{kpc}$.

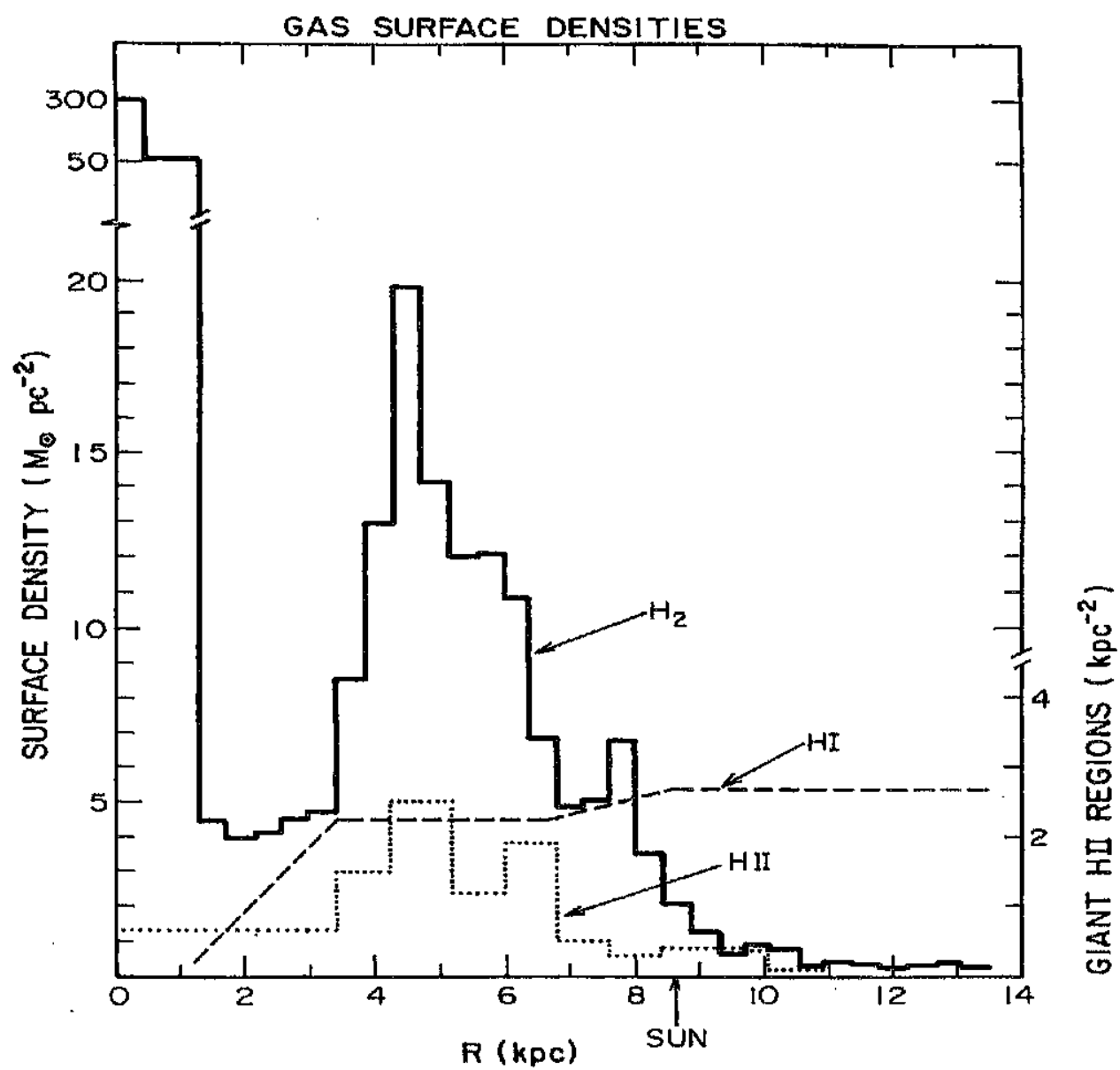

Fig. 8.2. The gas surface densities in the Milky Way disk are shown for $\mathrm{H}_{2}$ (Clemens et al. 1988), HI (Burton \& Gordon 1978) and giant HiI regions ( $>$ M 42). Both the $\mathrm{H}_{2}$ and $\mathrm{HI}$ values include a 1.36 correction factor for $\mathrm{He}$

Since the total Galactic star formation rate is $\sim 3 M_{\odot}$ per yr, the characteristic cycling time for $\mathrm{H}_{2}$ into new stars is $\sim 2 \times 10^{9} M_{\odot} / 3 M_{\odot} / \mathrm{yr} \sim 10^{9} \mathrm{yr}$. Thus, the $\mathrm{H}_{2}$ clouds are forming stars at a rate much slower than their freefall collapse time within the clouds $\left((G \rho)^{-1 / 2} \sim 3-5 \mathrm{Myr}\right)$ but on a timescale much shorter than the Galactic lifetime. Star formation is inefficient but a resupply of gas is required if star formation is not to die out in the next Gyr (which seems unlikely).

The smaller-scale distribution of $\mathrm{CO}$ emission indicates that the $\mathrm{H}_{2}$ gas resides in discrete clouds, rather than a diffuse, continuous medium. Along a typical line of sight in the Galactic plane there will be three to six 

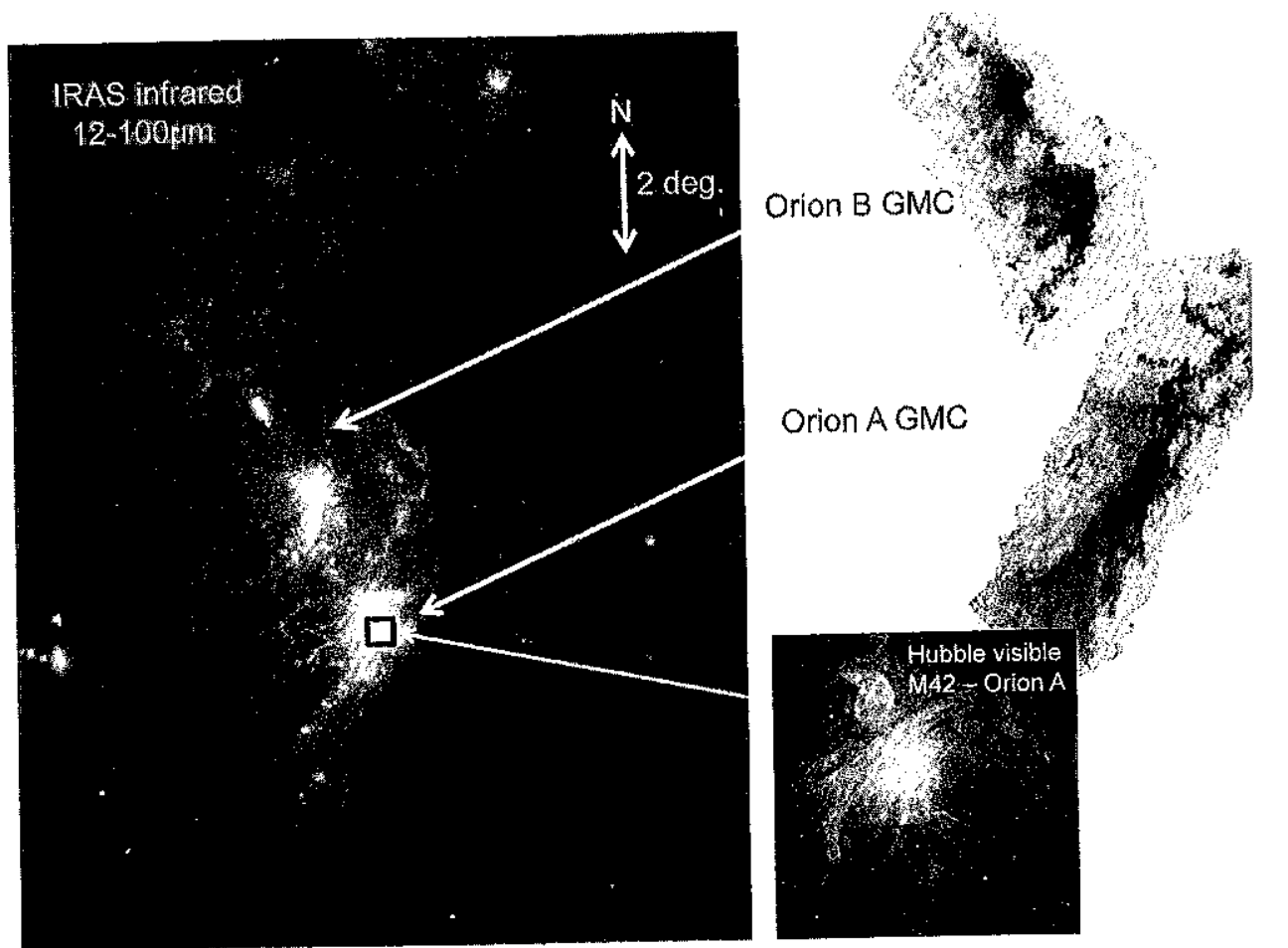

Fig. 8.3. The star-forming complex in Orion provides a close view of the complexity of both the ISM and the star formation activity. The left panel shows a very largescale image of the FIR emission as imaged by the IR astronomical satellite (IRAS) (Courtesy NASA/JPL-Caltech). The upper-right panel shows the integrated CO (1-0) line emission from the two Orion GMCs (Ripple et al. 2012). A Hubble Heritage image of the visible Hil region M42 is shown at the lower right - the visible HII region occupies a very small area of the Orion complex, as outlined in the IRAS image.

kinematically discrete CO emission features, occupying a small fraction of the overall permitted velocity range. Spatial mapping of the individual emission features indicates extends perpendicular to the line of sight typically 10 to $80 \mathrm{pc}$ - hence the name, Giant Molecular Clouds (GMCs).

The Orion GMC (actually two clouds) is the nearest GMC with an overall extent of $40 \mathrm{pc}$ (see Fig. 8.3) - in striking contrast to the optically visible Orion Nebula (M 42) for which the extent is $\lesssim 1$ pc! In the vicinity of M 42, the CO brightness temperature increases to a peak of $\sim 60 \mathrm{~K}$ (see Fig. 8.1, right), compared to $5-20 \mathrm{~K}$ in most of the rest of the cloud. These elevated temperatures are largely due to heating by the dust-embedded cluster of young stars in the Kleinmann-Low (KL) IR source, located behind the visible Trapezium/M 42 OB star cluster.

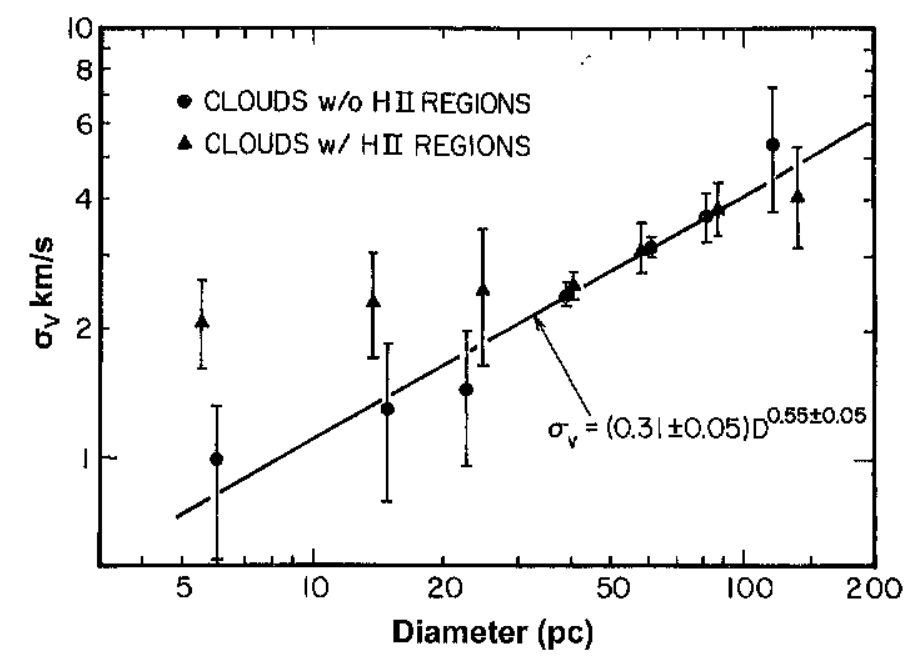

Fig. 8.4. The internal velocity dispersions of GMCs are shown as a function of diameter for clouds with and without giant Hrr regions, i.e., HII regions more luminous than M42 (Scoville et al 1987). This illustrates the empirical correlation found betweon (Scovd size and linewidth (the so-called size-lincwical correlation). The Her region at masses/sizes presumably due to feedback effects from massive star formation.

The gas heating associated with the KL nebula (and other IR sources associated with active star formation) occurs in two steps: heating of the embedding dust by absorption of photons emitted by the young stars, followed by $\mathrm{H}_{2}$ heating through collisions with the heated dust grains. Thermal equilibrium between the dust and the $\mathrm{H}_{2}$ is theoretically expected to occur at gas densities $n_{\mathrm{H}_{2}}>10^{4} \mathrm{~cm}^{-3}$ (Goldreich \& Kwan 1974). Indeed, the dust colour temperature in $\mathrm{KL}$ is $\sim 60 \mathrm{~K}$, i.e., similar to the gas kinetic temperature determined from the CO line brightness temperature.

\subsubsection{Self-gravitating GMCs}

One of the most perplexing and still not understood features of the GMCs is their large velocity dispersions, as determined from the width of the molecular emission lines. At gas temperatures of $10-80 \mathrm{~K}$, the thermal velocity dispersion. (sound speed) should be $\sim 0.1-0.3 \mathrm{~km} / \mathrm{s}$, yet the observed velocity dispersions are typically $1-4 \mathrm{~km} / \mathrm{s}$. The linewidths are correlated with cloud diameter, $D$. The observed relation is $\sigma_{\mathrm{V}}=0.31 D_{\mathrm{pc}}^{0.55} \mathrm{~km} / \mathrm{s}$ (see Fig. 8.4). Thus the kinetic energy in large-scale supersonic motions is $~ 100$ times the expected thermal energy. The containment of these supersonic turbulent motions is provided by the self-gravity of the molecular gas within each 
cloud. Independent estimates of the cloud masses using both dust extinction measurements and non-LTE analysis of the molecular lines, indicate approximately enough gas mass for the GMCs to be self-gravitating and in virial equilibrium with the observed internal motions. Nevertheless, it should be pointed out that although the kinetic energy and gravitational potential energy approximately balance, the GMCs do not have the expected spherical shapes; they are often elongated and have internal filamentary or sheet-like structures.

In any case, it is clear that the GMCs are not in pressure equilibrium with the external diffuse Hr and Hrr phases of the ISM. Their pressures are generally taken to be $n T=3000 \mathrm{~cm}^{-3} \mathrm{~K}$, i.e., a factor of 100 lower than the turbulence pressures within the GMCs as reflected in their internal velocity dispersions.

Given that the GMCs are self-gravitating, one can obtain their mean densities as a function of cloud diameter using the above empirical relation between the velocity dispersion and cloud diameters (i.e., the observed size-linewidth relation):

$$
\left\langle n_{\mathrm{H}_{2}}\right\rangle=180\left(\frac{D}{40 \mathrm{pc}}\right)^{-0.9} \mathrm{~cm}^{-3}
$$

where the scaling is to $40 \mathrm{pc}$ - the size at which half of the overall Galactic $\mathrm{H}_{2}$ mass is in clouds larger and half is in clouds smaller. The cloud mass distribution function is $N(M) \propto M^{-1.6}$. For a cloud of diameter $40 \mathrm{pc}$, the above relation implies a mass of $4 \times 10^{5} M_{\odot}$ (including the $36 \%$ mass contribution of $\mathrm{He}$ ).

\subsubsection{Molecular masses from $\tau \gg 1$ CO emission}

How is it that the emission in optically thick CO lines can be used to estimate the total mass of $\mathrm{H}_{2}$ in galaxies? Observers of extragalactic $\mathrm{CO}$ commonly make use of a so-called $X$-factor or $\alpha_{\mathrm{CO}}$ to translate measurements of CO emission into estimates of molecular gas masses - let us see how this is physically justifiable.

For a resolved cloud the integrated line brightness temperature, $L_{\mathrm{CO}}=$ $\int T_{\mathrm{B}} \mathrm{d} v$, can be integrated over the projected area of the cloud to yield a 'luminosity' $L_{\mathrm{CO}}=d^{2} \int I_{\mathrm{CO}} \mathrm{d} \Omega$ where $d$ is the distance to the cloud. Thus,

$$
L_{\mathrm{CO}}=T_{\mathrm{B}}(\mathrm{CO}) \Delta V \pi R^{2} \quad \mathrm{~K} \mathrm{~km} / \mathrm{s} \mathrm{pc}^{2}
$$

where $R$ is the radius of an assumed spherical cloud. For clouds in virial equilibrium, $\Delta V=(G M / R)^{1 / 2}$ and therefore,

$$
L_{\mathrm{CO}}=(3 \pi G / 4 \rho)^{1 / 2} T_{\mathrm{B}}(\mathrm{CO}) M_{\mathrm{GMC}},
$$

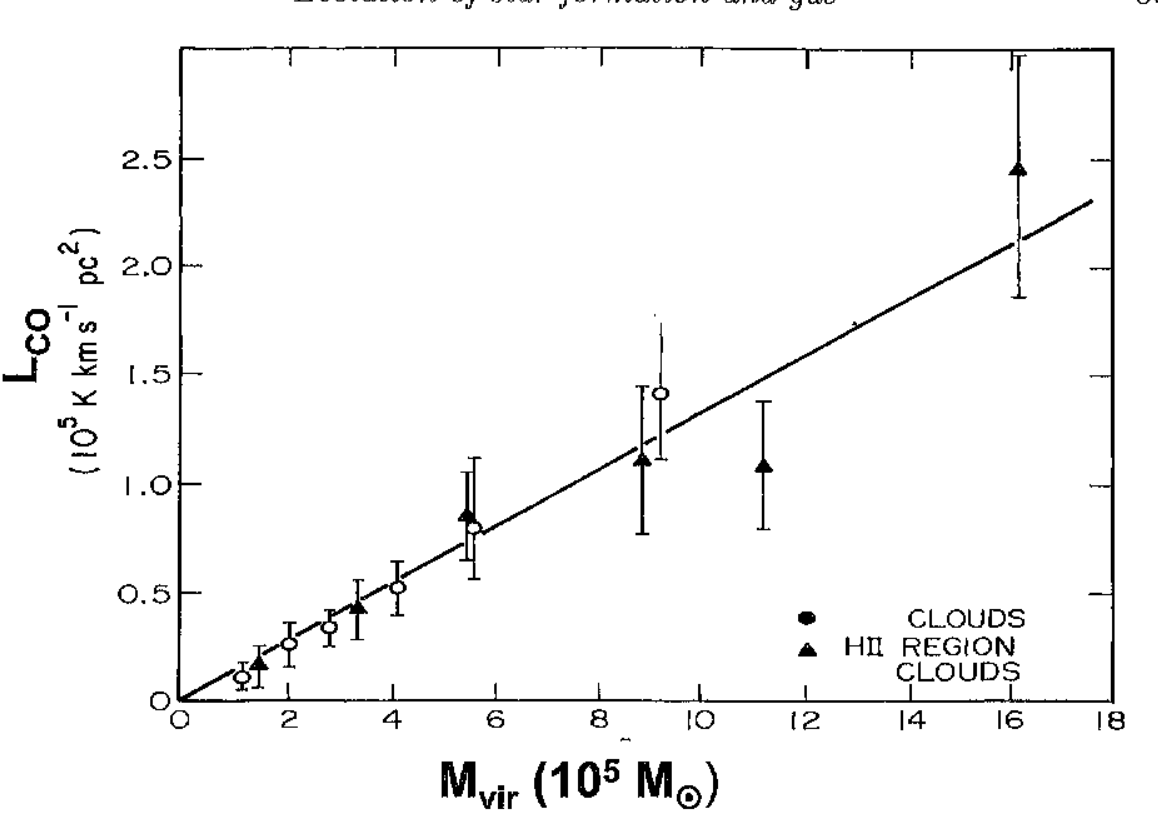

Fig. 8.5. The CO luminosities of GMCs are shown as a function of their virial masses for clouds with and without giant HiI regions (Scoville et al. 1987).

where $\rho$ is the mass density. Equation 8.5 predicts a linear scaling between cloud mass and the $\mathrm{CO}$ luminosity provided the clouds have approximately similar mean density and temperature. Equation 8.5 indicates that the constant of proportionality between $L_{\mathrm{CO}}$ and $M$ will vary as $T / \sqrt{\rho}$. Physically, what is going on here? Although the CO line is optically thick (and often thermalised), the increased mass of larger clouds gets reflected in an increased surface area emitting CO photons and an increased linewidth over which they are emitted.

Figure 8.5 illustrates the extremely good empirical correlation between measured CO luminosities and their virial masses (determined from resolved measurements of the size and linewidth). In fact, if the temperatures are determined by radiative heating of dust within the cloud (plus a low-level background heating by cosmic rays heating the gas to $\sim 10-15 \mathrm{~K}$ ), the overall range of mean temperatures is expected to be rather small. The dust radiatively cools as $\propto T_{\mathrm{D}}^{5-6}$ and it is hard to get the dust much above $40 \mathrm{~K}$ except in very localised regions of active star formation. Typical FIR colour temperatures of GMCs are in the range $20-40 \mathrm{~K}$ and only a few ultra-luminous IR galaxy (ULIRG) nuclei get up to $60 \mathrm{~K}$. Regions with elevated gas temperatures and hence high $T_{\mathrm{B}}(\mathrm{CO})$ are also likely to be denser (since their high temperatures probably reflect more concentrated star formation due to 
higher gas density). The effects of increased temperature and density will therefore partially compensate each other (see Equation 8.5).

For extragalactic observations where individual GMCs are not resolved, Equation 8.5 can still be used since the total CO luminosity is just the sum of that of the individual clouds, provided the GMCs do not overlap both on the sky and in velocity (unlikely given their small-volume filling factor in normal galaxies). In the extragalactic observations, the cloud diameters are not usually measurable but the unresolved apparent brightness temperatures vary as distance $d^{-2}$ so that the distance to the galaxy may replace $R$ in Equation 8.4. For the external galaxies, Equation 8.5 is translated to

$$
M_{\mathrm{H}_{2}}\left(M_{\odot}\right)=\alpha_{\mathrm{CO}} L_{\mathrm{CO}} \quad \mathrm{K} \mathrm{km} / \mathrm{s} \mathrm{pc}^{2},
$$

where $\alpha_{\mathrm{CO}} \simeq 4.9$ (Solomon \& Barrett 1991)

We should also note that for modest changes in metallicity (affecting the $\mathrm{CO}$ abundance relative to $\mathrm{H}$ ), $\alpha_{\mathrm{CO}}$ will change slowly (until $\mathrm{CO}$ becomes optically thin in large areas) - this is dramatically demonstrated by the fact that ${ }^{13} \mathrm{CO}$ luminosities of clouds are typically only a factor of $4-10$ times lower than those of $\mathrm{CO}$ despite the much lower abundance ratio of ${ }^{13} \mathrm{C} / \mathrm{C}$ $(1 / 40 \rightarrow 1 / 90)$. For the optically thick regime where photon trapping is important for the CO excitation, one can show analytically that the $\alpha_{\mathrm{CO}}$ should scale as CO abundance or metallicity $Z^{-0.4}$ (see Scoville \& Solomon 1974).

One instance where the above analysis must be modified is in a galactic nucleus if the gas is smoothly distributed in the central galactic potential Here, the CO linewidth will be determined not by the self-gravity of individual clouds but instead by that of the stars plus the gas, i.e., the linewidth will be larger than that associated with just the gas mass. There will then be more CO photons emitted per unit gas mass. For parameters associated with the most extreme cases in ULIRGs, the CO conversion factor may be reduced by factors of 2-5 (see Downes \& Solomon 1998 and Bryant \& Scoville 1999). In the photon trapping regime, the CO excitation temperature (and hence the brightness temperature) of the line will vary as the molecular abundance to the $\sim 0.4$ power (Scoville \& Solomon 1974). Thus, one expects that the CO line emissivity to mass conversion factor will vary with metallicity as $Z^{-0.4}$ at high redshift where the metallicities are generally lower.

\subsubsection{Lifetimes of GMCs}

Although much current theoretical analysis of star formation adopts the point of view that GMCs are relatively short-lived (10-30 Myr), I would like to provide several arguments why this can't be the case. These arguments were well known in the early days of molecular line astronomy but seem to have been overlooked in current discussions.

The first argument is simply based on the required conservation or continuity of ISM mass between the various ISM phases (HI, HII and $\mathrm{H}_{2}$ ). If the GMCs, or more specifically the $\mathrm{H}_{2}$ molecules, are short-lived then there must be cycling of gas between these phases. The mass flux from one phase to another and back must then be in equality (see Scoville \& Hersh 1979; Koda et al. 2009). This mass flux equality is given simply by

$$
\dot{M}_{\mathrm{H}_{2} \rightarrow \mathrm{HI}+\mathrm{HII}}\left(\equiv M_{\mathrm{H}_{2}} / \tau_{\mathrm{H}_{2}}\right)=\dot{M}_{\mathrm{HI}+\mathrm{HII} \rightarrow \mathrm{H}_{2}}\left(\equiv M_{\mathrm{HI}+\mathrm{HII}} / \tau_{\mathrm{HI}+\mathrm{HII}}\right),
$$

where we assume relatively little cycling to young stars (since that timescale was found to be $\sim 1$ Gyr above). In the interior of our Galaxy and most nearby spirals, the $\mathrm{H}_{2}$ dominates in mass compared to $\mathrm{HI}$ and $\mathrm{Hr}$ by a substantial factor (e.g., more than a factor of five in the Galactic molecular cloud ring). Thus if cycling between the phases is to be plausible at all (without disobeying mass conservation!) at these interior radii, the timescale for a molecule to remain a molecule must be correspondingly longer than the timescale for a particle in the diffuse phases (HI or HII) to remain atomic or ionic. The latter might be expected to be given by the dynamical time at density a few $\mathrm{H} \mathrm{cm}^{-3}\left(\sim 10^{8} \mathrm{yr}\right)$ or by the time needed to pass between the compressive spiral arms (also $\sim 2 \times 10^{8} \mathrm{yr}$ ). Inverting the mass continuity equation (Equation 8.7), one finds:

$$
\tau_{\mathrm{H}_{2}}=\frac{M_{\mathrm{H}_{2}}}{M_{\mathrm{HI}+\mathrm{HI}}} \times \tau_{\mathrm{HI}+\mathrm{HII}} \sim 5 \times\left(2 \times 10^{8}\right) \sim 10^{9} \mathrm{yr} .
$$

Thus, for cycling between the phases to work, the characteristic lifetime of a $\mathrm{H}_{2}$ molecule should be at least several times $10^{8} \mathrm{yr}$. And if there is no such cycling, then the timescale is even longer. Although this calculation was done for the interior of the Galaxy where $\mathrm{H}_{2}$ dominates $\mathrm{Hr}$, the estimate can be expected to hold elsewhere since it is in the interior of the Galaxy where there is the most disruptive feedback from star formation and other processes. In addition, the internal structure of the GMCs in the outer Galaxy is probably similar to that in the inner Galaxy (i.e., they are equally difficult to disrupt).

One must note that this is the timescale for a molecule to remain a molecule and not necessarily the timescale for a given GMC to retain its physical structure. Clouds may and probably do grow within the spiral arms and fragment into smaller clouds outside the arms - but the molecules cannot cycle all the way back to the diffuse HI or HII phases on a short timescale.

Another line of reasoning suggesting long GMC lifetimes is the fact that, in 


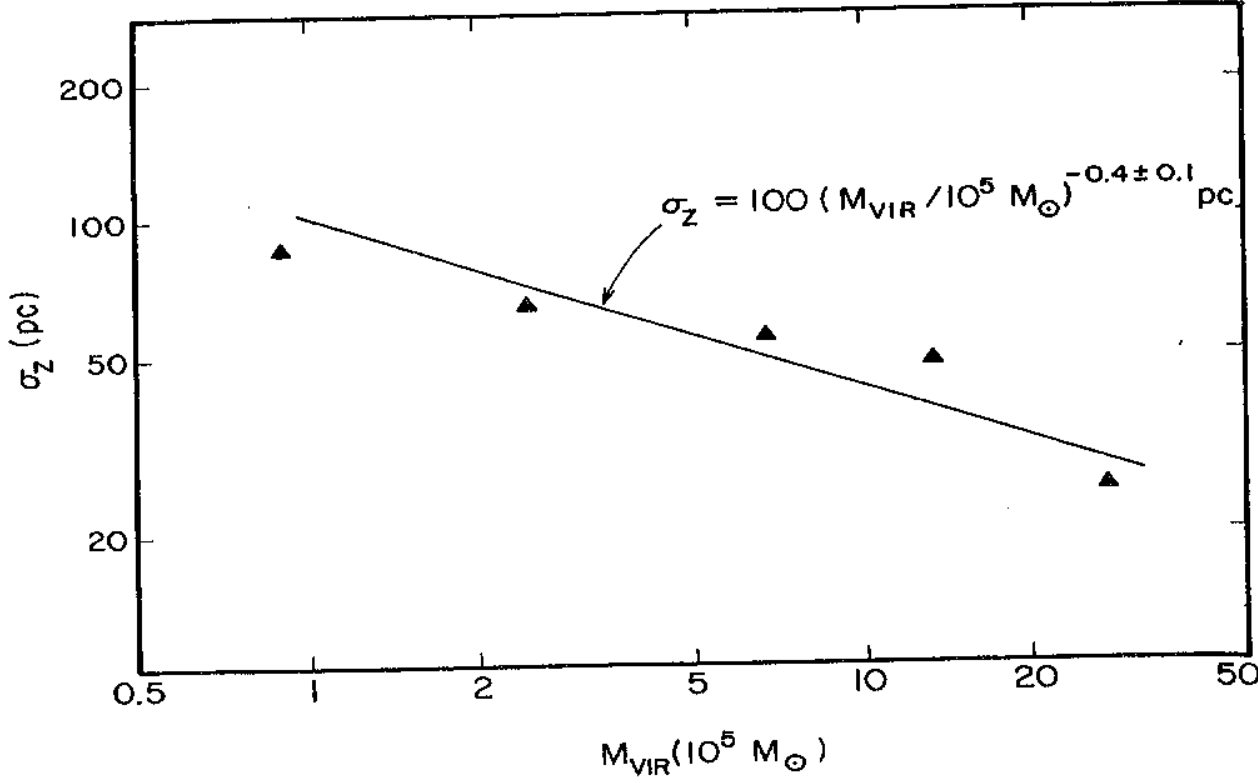

Fig. 8.6. The scaleheights of the GMC distribution perpendicular to the Galacti disk are shown as a function of cloud virial mass (Scoville et al. 1987). The nearly square-root dependence on cloud mass suggests an equipartition of the cloud kinetic ener last longer than several $10^{8} \mathrm{yr}$.

general, feedback processes from star formation within the GMCs are inefficient at disrupting the massive clouds (Scoville \& Hersh 1979; Scoville 2003 Murray et al. 2010). Most of the stellar feedback will be radiated and dissipated in shock fronts or leak out the cloud boundaries (as is surely the case in M 42). The total momentum needed to unbind the clouds is enormous, typically $\sim 5-10 \times 10^{5} M_{\odot} \mathrm{km} / \mathrm{s}$. Lastly, I point out that the scaleheights (perpendicular to the Galactic disk) of the GMCs as a function of their mass suggest that they have achieved approximate equipartition. In Fig. 8.6, the $z$-scaleheights of clouds indicate that $\sigma_{z} \propto M_{\mathrm{vir}}^{-0.4}$, i.e., approximately the $1 / 2$ power expected for equipartition. Equipartition requires that the clouds survive at least several cloud-cloud scattering times. The latter varies with the local Galactic space density of clouds but is almost always $\geq 10^{8} \mathrm{yr}$.

\subsubsection{GMC supersonic internal motions}

I mentioned earlier that the GMCs have highly supersonic internal motions as determined from the molecular line Doppler widths and that to date there is no satisfactory explanation. The long cloud lifetimes highlight this problem. The maps of molecular emission within individual clouds show that

the molecular emissions and their large velocity spread are fairly smooth across the projected area of the clouds, implying that the area covering factor is $\gtrsim 1$. If this is the case then the supersonic turbulent gas parcels will necessarily collide and dissipate their energy with the time it takes to cross the cloud diameter. This timescale is $40 \mathrm{pc} / 3 \mathrm{~km} / \mathrm{s} \sim 10^{7} \mathrm{yr}$. Thus the turbulent energy required to support the GMCs against gravitational collapse needs to be replenished within a similar timescale. (Reduction of the dissipation by postulating that magnetic fields are driving these motions requires very strong fields and does not change this estimate significantly since the collisional dissipation can still occur along the field direction.)

Although the feedback energy released from embedded young stars is of the right order of magnitude to replenish the turbulent energy, most of the energy released from the protostellar outflows is radiated away in highvelocity shock fronts and is also deposited on small length scales rather than scales comparable to the cloud size needed for the large scale support. Turbulent cascades generally transport energy from large scale to small scale (except in 2D turbulence, see Robertson \& Goldreich 2012) so it is difficult to see how the stellar feedback can maintain the turbulent energy.

A possible source of the turbulent energy might be large-scale 'corrugations' in the galactic plane mass distribution. It should be pointed out that despite the apparent virial equilibrium of the GMCs (in the sense that their gravitational potential energy is approximately twice their internal kinetic energy), the morphologies of the clouds do not appear virialised and spherical - this provides a strong argument that they are constantly being distorted by the external force gradients. Those associated with normal density wave spiral structure pass by only every $\sim 2 \times 10^{8} \mathrm{yr}$ and are therefore not sufficient. Jog \& Ostriker (1988) propose that GMC-GMC scattering is viable to maintain the internal motions but such scattering occurs also on a typical timescale of only every $\sim 10^{8} \mathrm{yr}$, which seems too long compared to the dissipation timescale.

D'Onghia et al. (2011) have recently suggested that many observed spiral structures may be stochastic in nature, where mass seeds corresponding to GMC masses (very likely the GMCs themselves) will induce a mass overdensity in the stellar disk via the swing-amplification process. Is it viable that these associated stellar disk mass enhancements actually feed back gravitational perturbation energy into the internal supersonic motions of the GMC gas? 
8.1.3.5 Summary

In summary, the observations of Galactic GMCs and local galaxies indicate that :

(a) The $\mathrm{CO}$ emission arises from discrete, self-gravitating clouds (GMCs).

(b) These GMCs have high internal supersonic motions such that their overall kinetic energy content is dominated by turbulence with effective pressure 100 times the gas thermal pressure and 100 times the pressure of the external diffuse ISM.

(c) The GMCs have a mass spectrum $N(M) \propto M^{-1.6}$ with the midpoint in the mass contributions at cloud diameter $\sim 40 \mathrm{pc}$ and mass $4 \times 10^{5} M_{\odot}$. For this size cloud, the mean density is $n_{\mathrm{H}_{2}} \sim 180 \mathrm{~cm}^{-3}-$ larger clouds have lower density and smaller clouds higher mean density.

(d) For such self-gravitating clouds with optically thick CO lines, the emitted CO luminosity is approximately proportional to the cloud mass with a constant of proportionality scaling as $T /(\rho)^{1 / 2}$ (i.e., $\alpha_{\mathrm{CO}}$ varying as $\rho^{1 / 2} / T$ ) and having much lower than linear dependence on metallicity.

(e) The lifetimes of the $\mathrm{H}_{2}$ and probably the GMCs are apparently larger than $10^{8} \mathrm{yr}$ and possibly $10^{9} \mathrm{yr}$ based on simple mass-conservation arguments in the ISM between the diffuse and dense phases. This is consistent with what one might expect based on the great inertia of the GMCs (i.e., resistance to disruption/dissociation) and the fact that their extraordinarily high effective internal pressures (compared to the external diffuse phases) makes it very difficult for pressure disturbances in the external medium to significantly influence their internal structure.

(f) The maintenance of the supersonic motions within GMCs, providing support The me replenished on the against collapse, remains unso timescale of a few $10^{7} \mathrm{yr}$, yet the clouds last $>10^{8} \mathrm{yr}$. Internal feedback from star formation deposits energy on length scales which are too small. The nonspherical shapes of the GMCs suggest large-scale external force gradients may be responsible, such as those associated with GMC-GMC scattering, clouds motions perpendicular to the galactic disk potential or the recently proposed stochastic spiral structure.

\subsection{Star formation}

Here I describe the various probes of star formation in galaxies and their correlation with the molecular gas contents. I devote considerable effort to developing an interpretive framework for the FIR emission from optically thick dust clouds.

\subsubsection{Probes of star formation}

There are a number of observational tracers which have been developed to measure the star formation rates (SFRs) in galaxies (see Calzetti, this volume). The Hi recombination lines in the visible and near-IR (e.g., H $\alpha$ and $\mathrm{P} \alpha$ ) have the fluxes proportional to the HII region emission measures, hence the OB star formation rate over the last $10^{7} \mathrm{yr}$. The restframe (far-) UV continuum ( $[\mathrm{F}] \mathrm{UV}$ ), at $\lambda<2000 \AA$, arising from hot, early-type stars has been used to infer the SFRs for large samples of galaxies. Both the emission lines and the UV continuum can be severely attenuated by dust extinction in star-forming regions. Even for the galaxies with detected UV continuum, the extinction corrections are often factors of 5-10! For the dust-obscured star formation, the FIR luminosity $\left(\lambda_{\text {rest }}=8-1000 \mu \mathrm{m}\right)$ provides a much more reliable measure of the SFR. These FIR SFRs can now be obtained for large samples of galaxies using observations from the Spitzer and Herschel space telescopes, albeit with relatively low angular resolution and sensitivity to SFR (compared to the UV). A summary of all these techniques, including relevant SFR equations, appears in the recent paper by Murphy et al. (2011) so I will not detail all of them here - instead I will focus on developing a physical understanding of the IR emission.

\subsubsection{Infrared emission}

The FIR emission from both star-forming and active galactic nucleus (AGN) sources arises from dust surrounding these sources which has been radiatively heated by absorption of the outflowing photons. Here I develop the logical steps for interpreting the IR emission since I have not seen this done systematically elsewhere.

The dust temperatures are determined by radiative equilibrium at distance $R$ from a central source of luminosity $L$, with

$$
4 \pi a_{\mathrm{d}}^{2}\left\langle\epsilon_{\nu}\right\rangle \sigma T_{\mathrm{d}}^{4}=\pi a_{\mathrm{d}}^{2}\left\langle\kappa_{\nu}\right\rangle L /\left(4 \pi R^{2}\right)
$$

where $a_{\mathrm{d}}$ and $T_{\mathrm{d}}$ are the dust grain radius and temperature, and $\left\langle\epsilon_{\nu}\right\rangle$ and $\left\langle\kappa_{\nu}\right\rangle$ are the dust emission and absorption efficiencies (shown in Fig. 8.7, left panel), weighted, respectively, by the Planck spectrum at the local dust temperature and that of the luminosity source heating the dust. If the emission efficiency varies as $1 / \lambda$, i.e., $\epsilon_{\nu} \propto T_{\mathrm{d}}$, then

$$
T_{\mathrm{d}} \propto \frac{L^{1 / 5}}{R^{2 / 5}}
$$



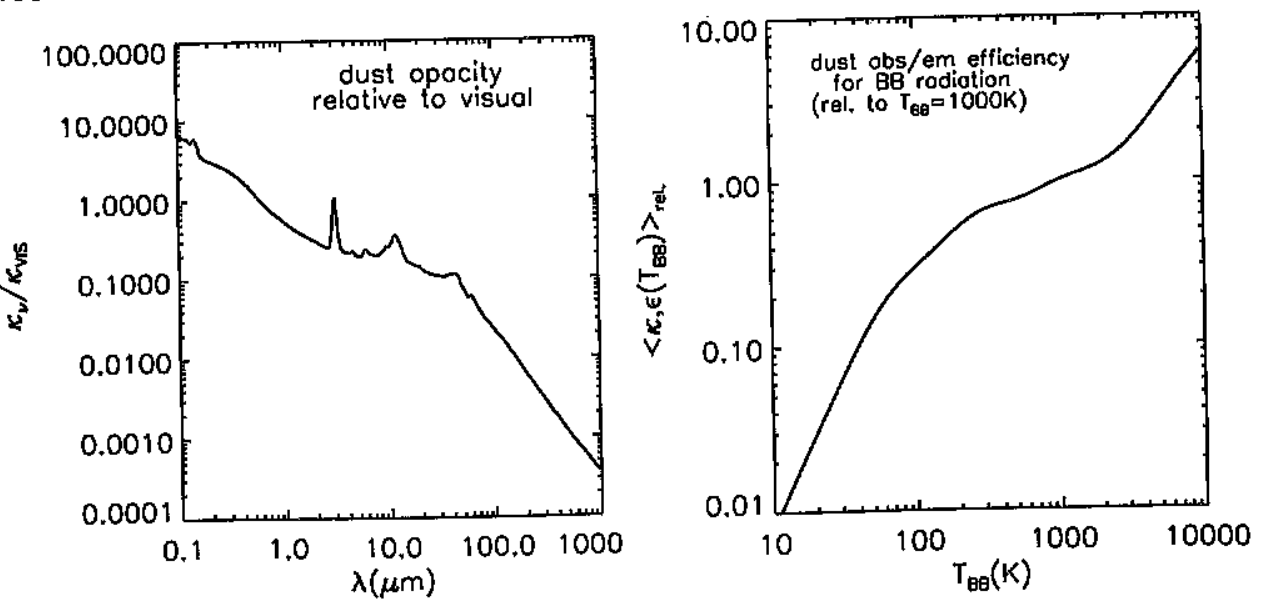

Fig 8.7. Left panel: the dust absorption opacity as a function of wavelength (Isella . 8. 2010) for al $201 \%$ fistribution $n(a) \propto a^{-3.5}$ and $61 \%$ water is normalised relative to from 0.01 to $1 \mu \mathrm{m}$ radius. The absorption coefict ${ }^{4} \mathrm{gr}^{-1}$ ) - for a standard gasthat at visual wavelength $\left(6060 \mathrm{~A}, \kappa V=2.3 \times 104{ }^{4}{ }^{2}-2\right.$ a standard gasto-dust abundance ( $\sim 100$ in mass) with $N_{\mathrm{H}+2 \mathrm{H}_{2}}=2 \times 10^{2} \mathrm{~cm}$ per mag of visual extinction $\left(A_{V}\right)$. An important leature of the extinction eurve is the relatively flat broadband absorption coefficient over the range $\lambda=2-50 \mu \mathrm{m}$. In the right panel, I show the Planck integrated absorption/emission coeficient as a function of blackbody temperature. Due to the 'flat' absorption coefficient in the nearand mid-IR (NIR/MIR), the Planck-integrated absorption and emission efficiency is quite in (Nopent opacity quite in

(Goldreich \& Kwan 1974). More generally,

$$
T_{\mathrm{d}} \propto \frac{L^{1 / 4}}{R^{1 / 2}}\left\{\frac{\left\langle\kappa_{\nu}\left(T_{L}\right)\right\rangle}{\left\langle\epsilon_{\nu}\left(T_{\mathrm{d}}\right)\right\rangle}\right\}^{1 / 4},
$$

where $\left\langle\kappa_{\nu}(T)\right\rangle$ and $\left\langle\epsilon_{\nu}(T)\right\rangle$ are shown in the right panel of Fig. 8.7. Due to the flatness of the broadband absorption coefficient from 2 to $50 \mu \mathrm{m}$, the grain absorption and emission efficiencies integrated over black-body spectra are decreasing only modestly from $T_{\mathrm{BB}}=1000-100 \mathrm{~K}$ (Fig. 8.7, right).

For an optically thin dust distribution surrounding a luminous source, the dust heating is mainly due to the central short-wavelength source, but for an optically thick dust envelope, the interior dust does not see the central source and is instead heated by secondary radiation. This secondary radiation, having longer wavelength than the central stellar or AGN source, is less tion, having longer wavelength the optically thick case will therefore be colder (than it would be if exposed to the shorter-wavelength primary photons of the central source). In very optically-thick cases, the $\left\langle\kappa_{\nu}\left(T_{L}\right)\right\rangle$ in Equation 8.8 can be evaluated approximately with $T_{L}(R) \sim T_{\mathrm{d}}(R)$, appropriately weighted over nearby radii.

Figure 8.8 shows the computed dust temperatures for the optically thin and optically thick cases, evaluated from Equation 8.11. In the opticallythick regime, the fall-off in dust temperature is $\propto r^{-1 / 2}-$ an extremely simple form due to the fact that the photons heating the grains and those emitted by the grains have similar wavelength distributions. One will therefore have $\langle\kappa\rangle \sim\langle\epsilon\rangle$ in the very optically-thick dust clouds.

The radial scale in Fig. 8.8 is for a central source luminosity of $10^{12} L_{\odot}$, appropriate to ULIRGs and submm galaxies (SMGs), but the radial distances can be scaled as $L^{1 / 2}$ for other luminosities (see Equation 8.11). Thus, these temperature profiles can be equally well applied to a dust cloud surrounding a luminous protostellar cluster of luminosity $10^{3}-10^{6} L_{\odot}$. The temperature profiles in Fig. 8.8 start at $\sim 1000 \mathrm{~K}$ which is a little below the dust sublimation temperatures $(\leq 1500 \mathrm{~K})$. Inside this radius, the dust will not survive. For a less luminous source, this inner radius will scale inwards; but the physical scales will all change by the same $L^{1 / 2}$ and the modelling remains homologous.

\subsubsection{Dust optical depth: $\tau<1$ or $\tau>1$ ?}

It is quite trivial to observationally distinguish the optically thick and thin sources since the latter will have a power-law flux distribution on the shortwavelength side of the peak whereas the former will appear more exponential (see Scoville \& Kwan 1976). (If the dust distribution is clumpy, photons can emerge from the inner regions with hot dust - thus, in optically-thick dust clouds, a non-exponential spectral energy distribution [SED] at short wavelengths might also occur; see Fig. 8.17.)

Virtually all IR sources associated with active star-forming regions (Galactic GMCs and starburst nuclei) are optically thick into the MIR (based on their sharp short wavelength fall-off). Since the dust opacity is fairly flat across the wavelength range $(2-50 \mu \mathrm{m})$, one then expects that the source will become optically thin only at $\lambda>50 \mu \mathrm{m}$, as long as it is optically thick at $3-10 \mu \mathrm{m}$. Thus, it is most appropriate to employ the optically thick dust temperature distribution shown as a solid line in Fig. 8.8 and fit numerically by

$$
T_{\mathrm{d}}=630 \frac{\left(L / 10^{12} L_{\odot}\right)^{1 / 4}}{R_{\mathrm{pc}}^{1 / 2}} \mathrm{~K} .
$$




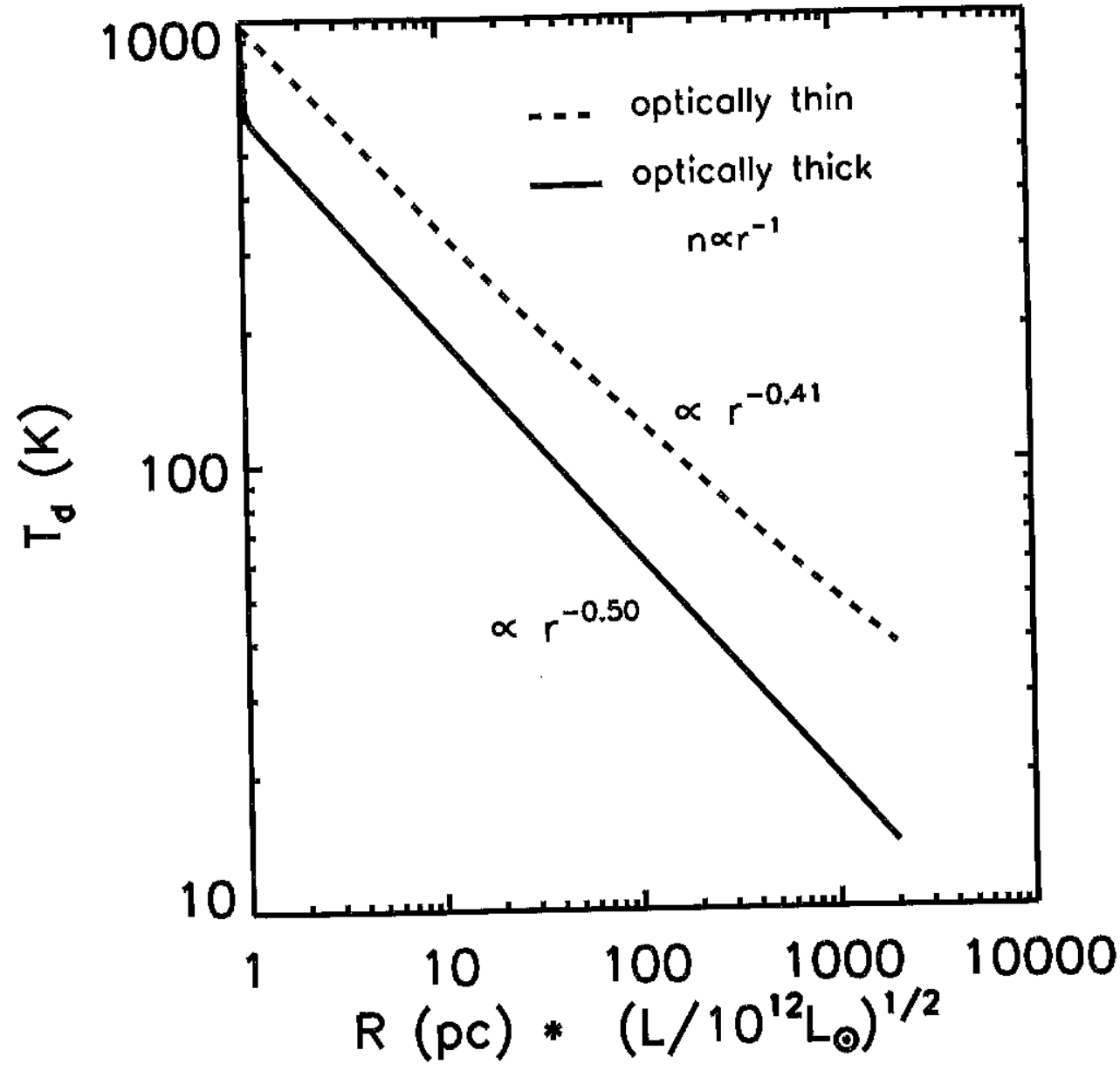

Fig 8. The temperature of dust heated by a central luminosity source is shown as a function of radius for optically thin dust (with all the heating provided by a $\sim 10^{4} \mathrm{~K}$ blackbody) and for very optically thick dust (where after the innermost radius, the heating is due to dust at the same radius and temperature). This radius, the hince the temperature gradients are resembles closely the vacial physical generally quite shallow. scalelengths will simply stretch homologously with changing sou higher or lower Thus the same curves can be used for sources with very much higher or lower luminosity. For optically-thick FIR dust emission the dust temperature varies as $T_{\mathrm{d}} \propto r^{-1 / 2}$. In real emission sources, the dust is optically thin rist at the inner boundary, optically thick at intermediate radil and then thin at the outer radii so the temperature profiles must be pieced together at the appropriate radii. (These equilibrium dust temperatures were calculated using the grain absorption coefficients shown in Fig. 8.7 and the inner boundary is taken to be where the coefin temperature is $1000 \mathrm{~K}$, i.e., somewhat below the expected dust sublimation temperatures.)
One can invert Equation 8.12 to find the characteristic size of the emitting region:

$$
R_{\text {thick }}=100\left(63 / T_{\mathrm{d}}\right)^{2}\left(L / 10^{12} L_{\odot}\right)^{1 / 2} \quad \mathrm{pc},
$$

if the total IR luminosity and dust temperature are known. The latter might be derived by fitting the MIR SED, or more crudely, from the wavelength of the peak. If the dust is opaque, then the standard Planck expression yields $T_{\mathrm{d}}=100 \times\left(51 \mu \mathrm{m} / \lambda_{\text {poak }}\right) \mathrm{K}$ but if the dust is optically thin, then the peak wavelength is reduced by a factor $\sim(3 /(3+\alpha))$ where $\alpha$ is the power-law index for the opacity, $\kappa_{\nu} \propto \nu^{\alpha}$, near the IR peak.

In practice, the opacity can never be much greater than unity at the peak. This is due to the simple fact that the luminosity cannot escape from the inner regions which have high opacity to the cloud surface. And once the outward luminosity flux has shifted to wavelengths where the opacity becomes less than unity, it escapes. This can be seen analytically using the fact that the emergent emission for a given grain is proportional to $B_{\nu} \epsilon_{\nu} \mathrm{e}^{\left(-\tau_{\nu}\right)}$. At $\lambda>70 \mu \mathrm{m}, \epsilon_{\nu} \propto \nu^{\sim 1.6}$. If $\tau_{\nu}=\left(\nu / \nu_{0}\right)^{1.6}$ (i.e., unity at $\nu=\nu_{0}$ ), then the emission of the grain will peak at $\nu_{\mathrm{m}} \simeq(3+1.6) \nu_{0} /\left(1.6+h \nu_{0} / k T_{\mathrm{d}}\right)$, where $\tau=\left(\nu_{m} / \nu_{0}\right)^{1.6}$. Thus, for example, with $T_{\mathrm{d}}=100 \mathrm{~K}$, the blackbody peak at $51 \mu \mathrm{m}$ is shifted to 78 and $395 \mu \mathrm{m}$ for $\lambda_{0}=102$ and $787 \mu \mathrm{m}$, respectively. At the peaks the optical depths are $\tau_{\text {peak }}=1.5$ and 3.0 in the two cases. This illustration was for an isothermal dust distribution with $\tau$ simplistically representing the foreground optical depth. This also provides a strong note of caution - if one is deriving the dust temperature from the wavelength of peak emission, the peak will usually be shifted to a longer wavelength where the dust starts to become transparent.

\subsubsection{Dust temperature of the emergent luminosity}

So how can the characteristic dust temperatures be derived? Probably the best approach, if the dust is believed to be optically thick on the short wavelength side of the peak (based on an 'exponential' rise), is to simply assume that $\tau \sim 1$ at the peak and estimate the temperature of the dust emitting the bulk of the emergent luminosity from $T_{\mathrm{d}} \gtrsim 100 \times\left(80 \mu \mathrm{m} / \lambda_{\text {peak }}\right) \mathrm{K}$. To improve on this ad hoc correction requires an assumption of the dust density distribution with radius and modelling the emergent SED (as is done below in Section 8.2.9).

For an optically-thick source, the emergent radiation at each wavelength will be from a depth where $\tau_{\lambda} \simeq 1$. Since the dust opacity falls off at longer wavelengths and the temperature is falling at larger radii, this implies, 


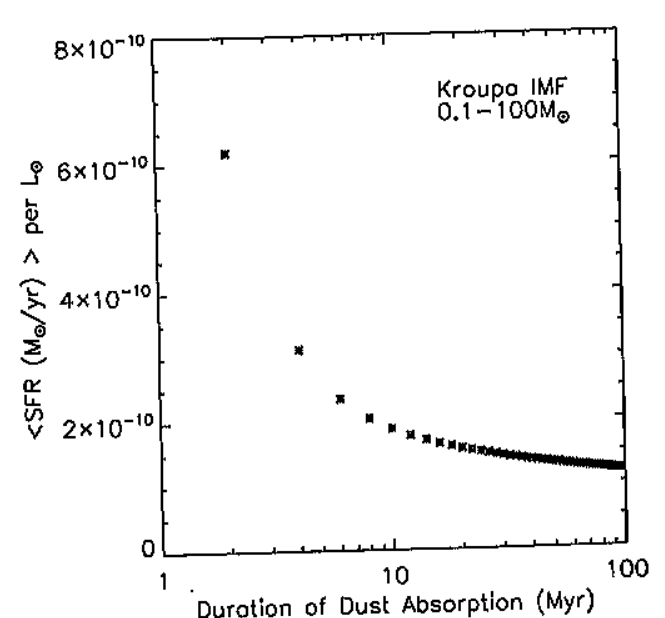

Fig. 8.9. The conversion of observed IR luminosities into estimates of the SFR depends on the duration time of the dust absorption. Here I have used Starburst99 models with a Kroupa IMF and assumed that all stellar and nebular photons longward of the Lyman limit are absorbed for the dust duration time (in Myr) and then none are absorbed after that. For normal star-forming regions this timescale is 10 Myr and for ULIRGs 50-100 Myr. If the dust envelope lasts less than $10 \mathrm{Myr}$ and or is only partially covering, then the conversion factor is significantly higher an exposed HII region like the Orion nebula.

somewhat counter-intuitively, that at wavelengths short of the peak wavelength, one will sample dust at increasingly larger radii, i.e., lower and lower temperatures!

\subsubsection{Star formation rate from $L_{\mathrm{IR}}$}

Derivation of SFRs from the IR luminosities is fairly straightforward and robust. This was first done by Scoville \& Young (1983) using the fact that the bulk of the luminosity from a stellar population at early times is generated largely by the OBA stars. For those stars an approximately fixed percentage (13\%) of their initial mass gets processed through the CNO cycle and one knows the energy produced per CNO process. This derivation is analogous to the 'fuel consumption theorem' of Renzini \& Buzzoni (1986).

A more precise, modern approach is to run starburst models (e.g., StarA meitherer et al. 1999) and integrate up the luminosity as a function burst99; Leitherer et al. 1999) and intial mass function (IMF), and its mass range, and decide which photons will be absorbed by dust and for mass range, and decide which photons how long the dust absorption persists. In Fig. 8.9 I how rest using Starburst99 with a Kroupa IMF $\left(0.1\right.$ to $100 M_{\odot}$, Kroupa 2001) as a function of the duration of the dust absorption. The latter quantity is probably $\sim 10^{7} \mathrm{yr}$ for Galactic star-forming regions but could be closer to $10^{8} \mathrm{yr}$ for merging starburst galaxies where the dust is more widely distributed. For this calculation, I assume all stellar and nebular photons longward of the Lyman limit ( $912 \AA$ ) are absorbed by the surrounding dust. From Fig. 8.9, one could reasonably compute a SFR given by

$$
\mathrm{SFR}_{\mathrm{IR}}=2-1.2 \times 10^{-10}\left(L_{\mathrm{IR}} / L_{\odot}\right) \quad M_{\odot} / \mathrm{yr}
$$

with the lower value being appropriate to the ULIRGs which have longer duration for the dust shrouding. The standard relation given by Murphy et al. (2011) corresponds to $1.5 \times 10^{-10} M_{\odot} \mathrm{yr}^{-1}\left(L_{\mathrm{IR}} / L_{\odot}\right)$. The simpler derivation outlined above (based on the CNO cycle energy production) is quite similar to Equation 8.14 after one corrects for the mass going into non-OBA stars for a Kroupa IMF.

\subsubsection{Dust and ISM mass estimates}

On the long-wavelength Rayleigh-Jeans (R-J) tail of the FIR emission, the dust will be optically thin and the observed continuum fluxes provide an excellent means of determining the overall mass of dust. If the dust-to-gas abundance is normal, this dust mass can then be scaled to estimate the overall mass of ISM within a star-forming region or a distant galaxy.

On the optically-thin R-J tail of the IR emission, the observed flux density is given by

$$
F_{\nu}=\kappa_{\nu} T_{\text {dust }} \nu^{2} M_{\text {dust }} /\left(4 \pi d_{l}^{2}\right),
$$

or in terms of the dust opacity per unit gas mass, $\kappa_{\nu}(\mathrm{ISM})=\kappa_{\nu} \times$ $M_{\text {dust }} / M_{\text {ISM }}$,

$$
F_{\nu}=\kappa_{\nu}(\mathrm{ISM}) T_{\mathrm{dust}} \nu^{2} M_{\mathrm{rSM}} /\left(4 \pi d_{1}^{2}\right),
$$

where $d_{1}$ is the source luminosity distance. In normal star-forming galaxies, the majority of the dust is at $\sim 20-25 \mathrm{~K}$, and even in the most vigorous starbursts like Arp 220 the FIR/submm emission is dominated by dust at temperatures $\leq 45 \mathrm{~K}$. Thus the expected variations in $T_{\text {dust }}$ have less than a factor two effect on the observed flux.

The dust opacity per unit mass of total ISM gas, $\kappa_{\nu}$ (ISM) in Equation 8.16 , can be calibrated from the extensive submm observations of nearby galaxies. Seventeen of the nearby SINGS survey (Spitzer Infrared Nearby Galaxies Survey; Kennicutt et al. 2003) galaxies have good total submm flux measurements obtained with the SCUBA instrument, mounted at the James 

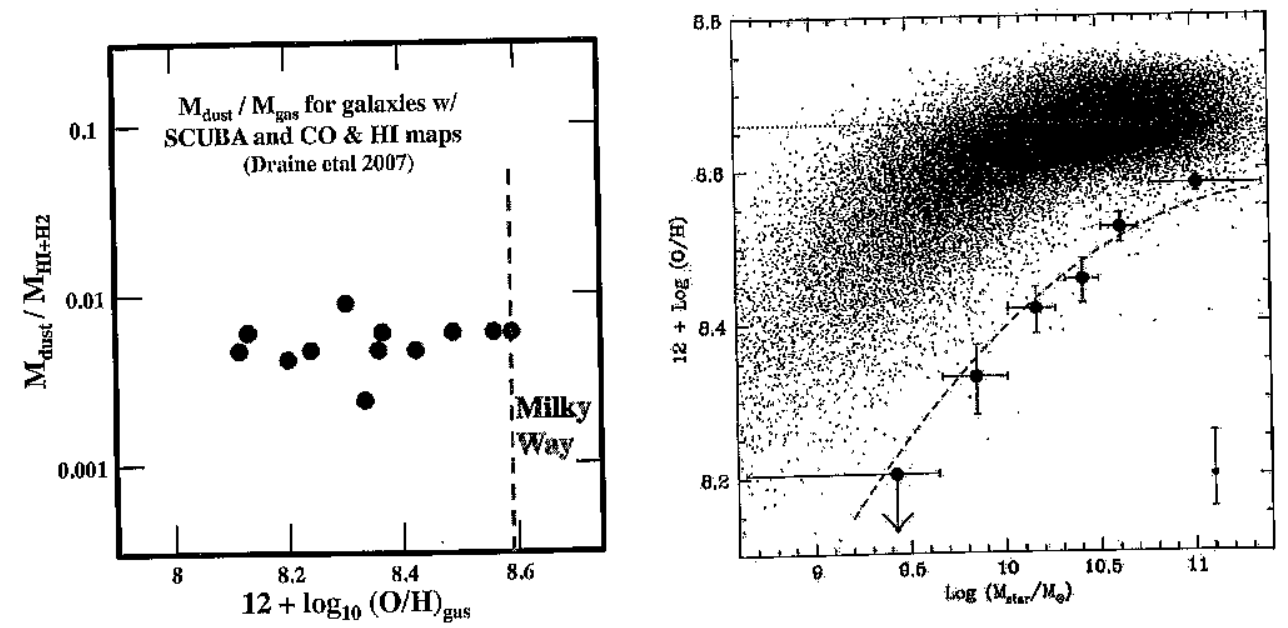

Fig. 8.10. Left panel shows the dust-to-gas mass ratios derived by Draine et al. (2007) for (2007) for of the $\mathrm{H}_{2}$ and galaxies with both Hr gas; right panel shows the et al. (2006). points) and binned values for $z \sim 2$ gadaxies (red d Milky Way there is little Over a range of 0.5 dex in metalicity below that of the Milky Way there is little variation in the dust-to-gas ratios. Since the galaxies selected here are massive, their metallicities even at $z=2$ are expected to be within this range based on emission line ratios. Lower-metallicity irregular galaxies probably do show a decrease in the dust abundance (see Draine et al. 2007).

Clerk Maxwell Telescope, at $850 \mu \mathrm{m}$ (see Draine et al. 2007), as well as good measurements of the total molecular $\left(\mathrm{H}_{2}\right)$ and atomic $(\mathrm{HI})$ gas masses.

In Fig. 8.10, the derived dust-to-gas $\left(\mathrm{H}_{2}+\mathrm{HI}\right)$ mass ratios from Draine $e$ al. (2007) are shown for the galaxies having SCUBA $850 \mu \mathrm{m}$ measurements, for a range of spiral type (Sa to $\mathrm{Sd}$ ) and as a function of mean metallicity. (Equivalent data for low-redshift elliptical galaxies are not available from Draine et al. 2007 due to lack of gas mass measurements in the early type galaxies.)

Figure 8.10 shows that over a range of $\sim 0.5$ dex in metallicity, there is little empirical evidence of variation in the dust-to-gas mass ratios and hence the submm flux. If one includes even lower-metallicity galaxies that do not have SCUBA $850 \mu \mathrm{m}$ fluxes (hence the submm fluxes must be extrapolated from an overall SED fit using shorter-wavelength observations), there is evidence for a metallicity dependence in the dust-to-gas ratio. Lastly, it should be emphasised that although some variations in the ratio of submm flux to ISM mass may be expected, ISM mass estimates at $\sim 30 \%$ accuracy (see
Table 8.1. Low-z galaxies with submm \& ISM data

\begin{tabular}{cccccc}
\hline \hline Galaxy & $\begin{array}{c}\text { Distance } \\
(\mathrm{Mpc})\end{array}$ & $\begin{array}{c}\mathrm{S}_{\nu}(450 \mu) \\
(\mathrm{Jy})\end{array}$ & $\begin{array}{c}\mathrm{S}_{\nu}(850 \mu) \\
(\mathrm{Jy})\end{array}$ & $\begin{array}{c}\log M_{\mathrm{HI}} \\
\left(M_{\odot}\right)\end{array}$ & $\begin{array}{c}\log M_{\mathrm{H}_{2}} \\
\left(M_{\odot}\right)\end{array}$ \\
\hline NGC 4631 & 9.0 & 30.7 & 5.73 & 9.2 & 9.5 \\
NGC 7331 & 15.7 & 18.5 & 2.98 & 9.4 & 9.7 \\
NGC 7552 & 22.3 & 20.6 & 2.11 & 9.7 & 10.0 \\
NGC 598 & 76.0 & 2.3 & 0.26 & 9.8 & 10.1 \\
NGC 1614 & 62.0 & 1.0 & 0.14 & 9.7 & 10.0 \\
NGC 1667 & 59.0 & 1.2 & 0.16 & 9.3 & 9.6 \\
Arp 148 & 143.0 & 0.6 & 0.09 & 9.9 & 10.2 \\
1ZW107 & 170.0 & 0.4 & 0.06 & 10.0 & 10.3 \\
Arp 220 & 79.0 & 6.3 & 0.83 & 10.0 & 10.3 \\
12112+0305 & 293.0 & 0.5 & 0.05 & 10.3 & 10.6 \\
Mrk 231 & 174.0 & 0.5 & 0.10 & 9.8 & 10.1 \\
Mrk 273 & 153.0 & 0.7 & 0.08 & 9.9 & 10.2 \\
\hline
\end{tabular}

Fig. 8.10) are still likely to be at least as accurate as those from CO line measures and much quicker, enabling large samples to be analysed.

In view of the large uncertainty in the submm absorption coefficient $\kappa$ and its scaling with frequency, we adopt an empirical approach based on submm observations of local galaxies where $\mathrm{HI}$ and $\mathrm{H}_{2}$ masses have been estimated. For the local galaxies on which to base this empirical approach, it is vital that both the submm fluxes and ISM masses are global values. In addition as a check on the reliability of the submm measurements we require two long wavelength flux measurements so one can check if there is reasonable consistency with expected values of the spectral index $\beta$.

For the spectral index $\beta$ of the R-J tail $\left(\mathrm{S}_{\nu}\right.$ varying as $\left.\nu^{\beta}\right)$, the observed flux ratios of submm galaxies can vary between 3 and 4 . For most dust models the spectral index of the opacity is typically 1.5 to 2 , implying $\beta=3.5$ to 4 . Empirical fits to the observed long wavelength SEDs give $\beta=3.5$ to 4 (Dunne \& Eales 2001; Clements et al. 2010) for local galaxies. For high- $z$ submm galaxies, the spectral index can be between 3.2 and 3.8 , but in most cases the shorter-wavelength point is getting close the IR peak in the restframe and therefore not strictly on the R-J tail. In the following, we adopt $\beta=3.8$.

Table 8.1 lists local spiral or star-forming galaxies for which both 450 and $850 \mu \mathrm{m}$ measurements exist with good signal-to-noise and for which global fluxes were estimated.

The $850 \mu \mathrm{m}$ fluxes were then converted to specific luminosity $L_{\nu(850)}$, using

$$
L_{\nu}=10^{-23} 4 \pi d_{L}^{2} S_{\nu}(\mathrm{Jy})=1.19 \times 10^{27} S_{\nu}(\mathrm{Jy}) d_{L}^{2}(\mathrm{Mpc}) .
$$




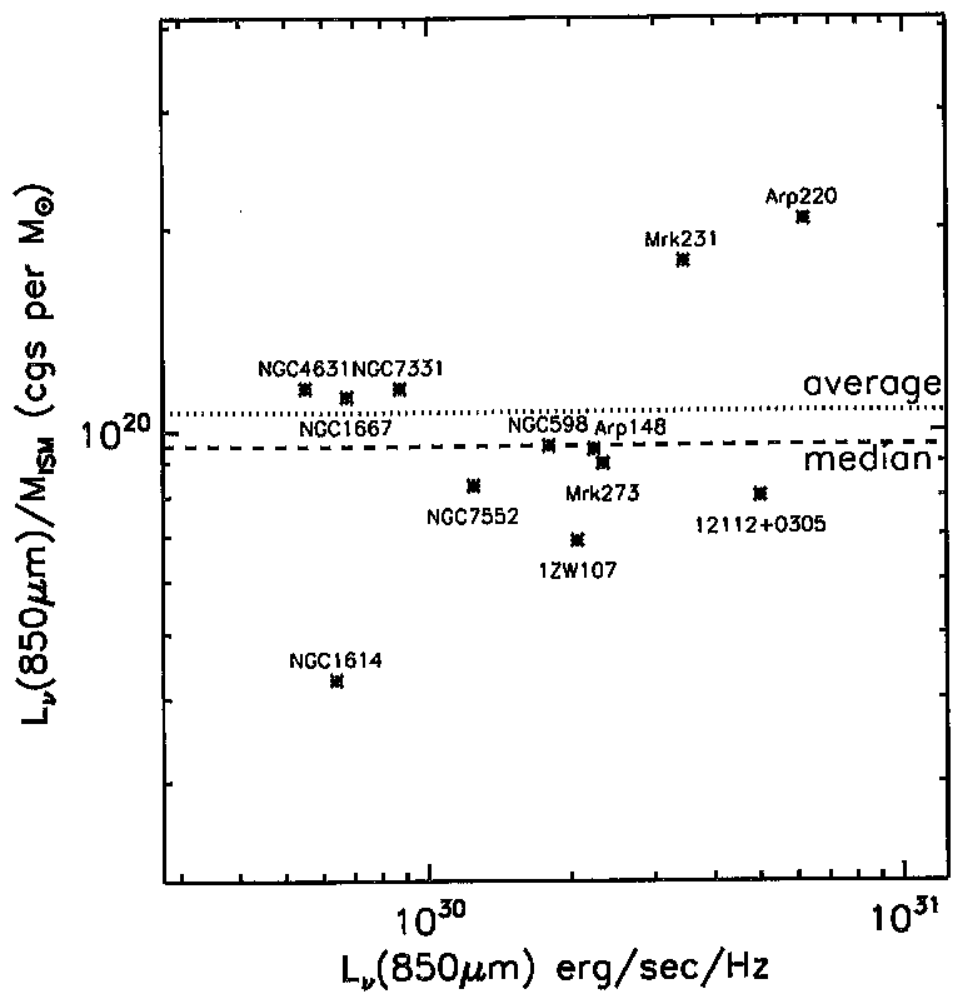

Fig. 8.11. The ratio of $L_{\nu}$ at $850 \mu \mathrm{m}$ to $M_{\text {ISM }}$ is shown for a sample of low $z$ spiral and starburst galaxies from Dale et al. (2005) and Clements et al. (2010). The average and median values for the sample are shown by horizontal lines.

Figure 8.11 shows the ratio $L_{\nu_{850}} / M_{\mathrm{ISM}}$ as a function of $L_{\nu_{850}}$ where $M_{\mathrm{ISM}}=M_{\mathrm{HI}}+M_{H_{2}}$. Based on this plot, we then adopt as a working value

$$
\frac{L_{\nu_{850}}}{M_{\mathrm{ISM}}}=1 \times 10^{20} \mathrm{erg} / \mathrm{s} / \mathrm{Hz} / M_{\odot}
$$

Define this mean value as

$$
\alpha_{850}=1 \times 10^{20} \mathrm{erg} / \mathrm{s} / \mathrm{Hz} / M_{\odot} .
$$

For high-redshift observations,

$$
\text { observed : }\left(\nu_{\text {obs }} S_{\nu_{o b s}}\right)=\text { restframe }:\left(\nu_{\text {rest }} \frac{L_{\nu_{\text {rest }}}}{4 \pi d_{L}^{2}}\right) \text {, }
$$

with

$$
\nu_{\text {rest }}=\nu_{\mathrm{obs}} \times(1+z)
$$

Therefore,

$$
\begin{aligned}
S_{\nu_{\mathrm{obs}}} & =\frac{\nu_{\text {rest }}}{\nu_{\mathrm{obs}}} \frac{L_{\nu_{\text {rest }}}}{4 \pi d_{L}^{2}} \\
& =(1+z) \frac{L_{(\perp+z) \nu_{\mathrm{obs}}}}{4 \pi d_{L}^{2}}
\end{aligned}
$$

with

$$
L_{\nu_{\mathrm{rest}}}=\alpha_{850}\left((1+z) \frac{\nu_{\mathrm{obs}}}{350 \mathrm{GHz}}\right)^{\beta} M_{\mathrm{ISM}}
$$

Then,

$$
S_{\nu_{\mathrm{o}}}=\frac{1+z}{4 \pi d_{L}^{2}} \alpha_{850}\left(\frac{(1+z) \nu_{\mathrm{obs}}}{350 \mathrm{GHz}}\right)^{\beta} M_{\mathrm{ISM}}
$$

where the $350 \mathrm{GHz}$ is the frequency corresponding to $850 \mu \mathrm{m}$.

Normalising to $M_{\mathrm{ISM}}=2 \times 10^{10} M_{\odot}$ with $\beta=3.8$,

$$
\begin{gathered}
S_{\nu_{\mathrm{obs}}}=\alpha_{850} 2 \times 10^{10} \times \frac{M_{\mathrm{ISM}}}{2 \times 10^{10}}(1+z)^{4.8}\left(\frac{\nu_{\mathrm{obs}}}{350 \mathrm{GHz}}\right)^{3.8} \frac{1}{4 \pi d_{L}^{2}}, \\
S_{\nu_{\mathrm{obs}}}(\mathrm{mJy})=1.67 \frac{M_{\mathrm{ISM}}}{2 \times 10^{10}}(1+z)^{4.8}\left(\frac{\nu_{\mathrm{obs}}}{350 \mathrm{GHz}}\right)^{3.8} \frac{1}{d_{L}^{2}(\mathrm{Gpc})},
\end{gathered}
$$

at $z=0.3,1,2$ and $3, d_{L}(\mathrm{Gpc})=1.5,6.6,15.5$ and $25.4 \mathrm{Gpc}$.

Figure 8.12 shows the new predicted fluxes as a function of redshift for both Band $6(240 \mathrm{GHz})$ and Band $7(347 \mathrm{GHz})$.

For reference, looking at the ALMA exposure time calculator, with $7.5 \mathrm{GHz}$ bandwidth in each polarisation and $10.2 \mathrm{~min}$ of integration at $\mathrm{Cy}$ cle 1 yields $1 \sigma=0.075 \mathrm{mJy}$ at $345 \mathrm{GHz}, 1 \sigma=0.042 \mathrm{mJy}$ at $240 \mathrm{GHz}$ and $0.029 \mathrm{mJy}$ at $100 \mathrm{GHz}$. The expected fluxes on Fig. 8.12 for $z=2$ are $\sim 1.7$ and $0.3 \mathrm{mJy}$ respectively for $2 \times 10^{10} M_{\odot}$. Thus Band 7 is optimal since the expected flux ratio is $\sim 5: 1$. Band $3(100 \mathrm{GHz})$ is not plotted in the figure since its expected flux density is 28 times below that of Band 6 .

To compare with the ability to detect $\mathrm{CO}$, we might use the source BX 691 from Tacconi et al. (2010) which has a $M_{*}=7.6 \times 10^{10} M_{\odot}$ and $M_{\mathrm{H}_{2}}=3.5 \times 10^{10} M_{\odot}$ at $z=2.19$ and $\mathrm{CO}(3-2)=0.15 \mathrm{Jy} \mathrm{kms}^{-1}$. For a width of $300 \mathrm{kms}^{-1}$, this has an average line flux of $0.5 \mathrm{mJy}$. If the mass is scaled to $2 \times 10^{10} M_{\odot}$, then the average line flux is $0.28 \mathrm{mJy}$. To get $5 \sigma$ or $0.056 \mathrm{mJy}$ sensitivity in a single $300 \mathrm{kms}^{-1}$, requires 5 hours!

In summary, measurement of the $R$ - $J$ tail of the emission (and using Equation 8.26) thus provides an excellent and fast means of determining dust and ISM masses in high-redshift galaxies using ALMA. (The coefficient in Equation 8.26 was derived empirically from submm observations and therefore 


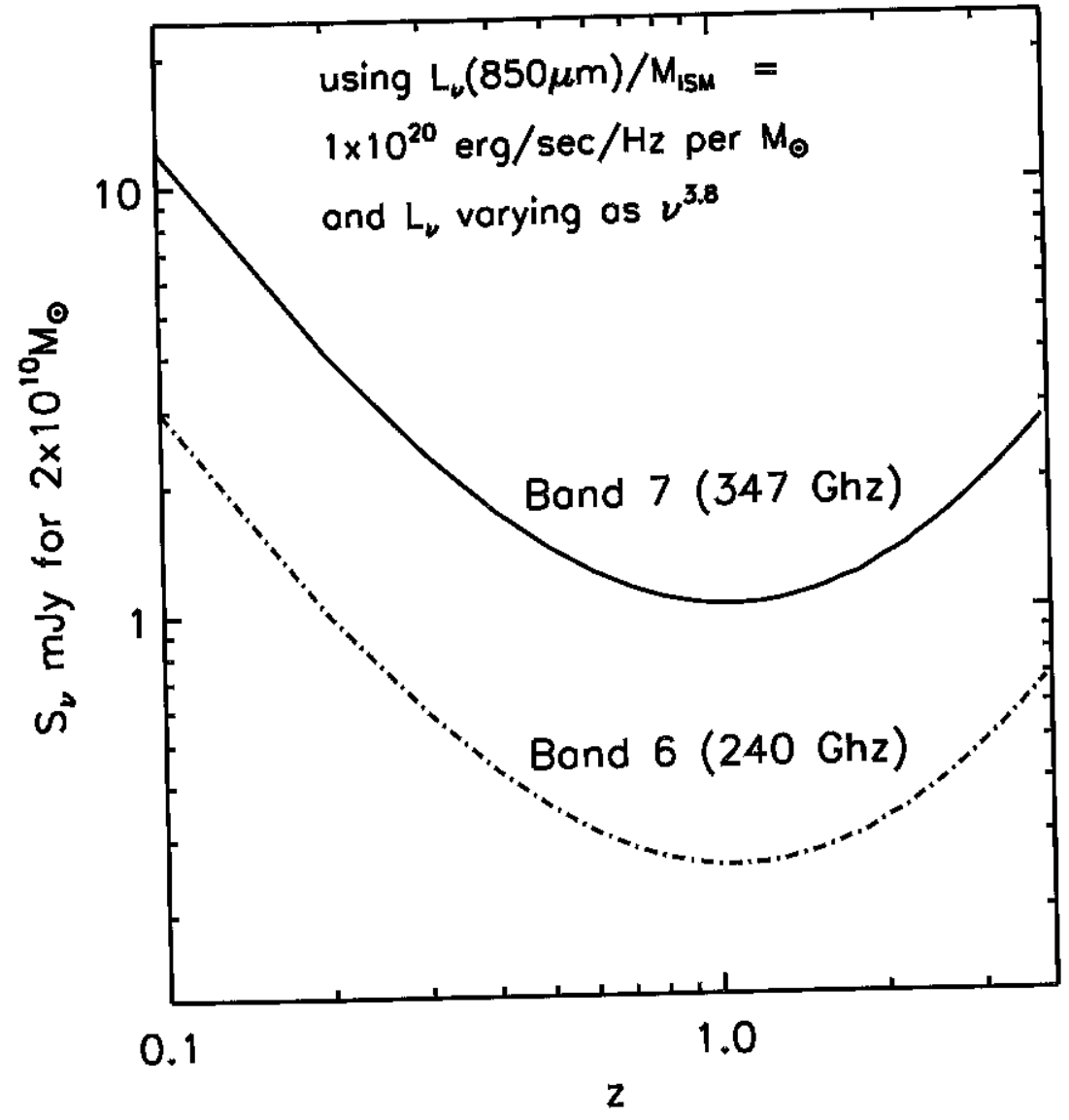

Fig. 8.12. The expected ALMA Band $6(240 \mathrm{GHz})$ and Band $7(345 \mathrm{GHz})$ flux ig. 8.12 . The shown for $M_{\text {ISM }}=2 \times 10^{10} M_{\odot}$.

may be slightly different than that obtained from the dust opacity shown in Fig. 8.7.)

\subsubsection{Effective source size}

For optically thick IR sources, one can estimate the effective size of the emitting region from

$$
R=\left(\frac{L}{4 \pi \sigma T_{\text {dust }}^{4}}\right)^{1 / 2}
$$

and scaling to ULIRG luminosities, we obtain

$$
R_{\mathrm{kpc}}=1.09 \times\left(\frac{L /\left(10^{12} L_{\odot}\right)}{\left(T_{\text {dust }} / 35 \mathrm{~K}\right)^{4}}\right)^{1 / 2} \mathrm{kpc} .
$$

This effective radius is the overall size of the optically-thick region emitting the FIR luminosity. In the event that the emission is optically thin, then the estimates from Equation 8.28 are of course lower limits. (In Section 8.2.9 optically-thick, radiative transfer modelling of the dust emission for a $r^{-1}$ dust density distribution is presented. Figure 8.15 shows the effective radius and dust temperature for the emitting region producing the majority of the emergent flux - for comparison with Equation 8.28.)

For local ULIRGs the typical FIR colour temperatures are $\sim 50 \mathrm{~K}$ so the IR emission radius is $\sim 500 \mathrm{pc}$ for $10^{12} L_{\odot}$. This estimate is similar to the overall size of the central concentration in Arp 220 (see below), indicating that the optically thick assumption is not unreasonable. The most luminous SMGs observed at high redshift can have $L_{\mathrm{IR}}>10^{13} L_{\odot}$; for these sources, the emission must come from galactic-scale regions, not just a compact nucleus.

For the Milky Way the FIR luminosity is $\sim 10^{10} L_{\odot}$ and the mean dust temperature $\sim 30-35 \mathrm{~K}$; the effective emitting radius from Equation 8.28 is $\sim 100 \mathrm{pc}$. However, this emission clearly originates from a large number of separate clouds, and the mean size of each must be $n_{\text {cloud }}^{1 / 2}$ times smaller. For example, if the Galactic emission is assumed to be contributed by $\sim 400$ IRluminous GMCs, then the effective size of the IR-dominant region in each would be $\sim 5$ pc.

\subsubsection{Luminosity and SFR estimates from submm continuum}

Estimating the FIR luminosity (and hence the SFR) from measurements on the submm R-J tail is an extremely questionable procedure - this hasn't stopped observers from routinely doing it! As noted above the R-J flux provides a measure of the dust mass weighed linearly by $T_{\mathrm{d}}$, but inferring a total bolometric luminosity requires knowing where the FIR peaks, and the fluxes near the peak. Observations near the SED peak can now be done using Herschel PACS (Photodetector Array Camera and Spectrometer) and SPIRE (Spectral and Photometric Imaging REceiver) but many of the SMGs are subject to source confusion at the SPIRE resolution. In the absence of direct observations at the SED peak, one must assume a dust temperature $(30-50 \mathrm{~K})$ and optical depth, perhaps based on the submm flux. For many of the SMGs, FIR luminosities in the range $10^{13-14} L_{\odot}$ have been estimated, implying SFRs of several $\times 10^{3} M_{\odot}$ per yr (typically assuming $T_{\mathrm{d}} \sim 30-50 \mathrm{~K}$ ) but such estimates must be extremely uncertain since the derived luminosity will vary approximately as $T^{4-5}$. Typical ISM masses of the SMGs derived from $\mathrm{CO}$ measurements or the submm continuum are $\sim 1-3 \times 10^{10} M_{\odot}$, 
implying that the ISM will be used up in star formation in an implausibly short time of $\sim 10^{7} \mathrm{yr}$.

Blain et al. (2003) attempted to derive empirically a scaling between the $850 \mu \mathrm{m}$ flux and $L_{\mathrm{IR}}$ based on the apparent $T_{\mathrm{d}}$ from fitting the SEDs of local galaxies. Unfortunately, there is large scatter in the correlation. In faw earlier, the notion of a single $T_{\mathrm{d}}$ is extremely shaky - both because there clearly is a range of temperatures and more importantly, the apparent $T_{\mathrm{d}}$ (derived from fitting near the FIR peak) is somewhat degenerate with the dust opacity (which also can cause an exponential fall-off to short wavelengths).

To summarise - it should be clear that one cannot constrain $T_{\mathrm{d}}$ without observations near the SED peak; and if one has such observations, it would be best to simply use them directly to estimate the luminosity. (Of course, if the observed source is at redshift $z>5$, then the submm flux measurements are in fact probing near the restframe SED peak; they can then provide a decent luminosity estimate.) In the next section, we model the opticallythick dust sources in order to appreciate some of the systematics and the range of uncertainty.

\subsubsection{Modelling optically-thick dust clouds}

For internally-heated FIR sources, it is vital to appreciate that the sources hor internally-heated essentially two totally independent parameters: the luminosity $L$ of the central heating source and the total mass of dust in the surrounding envelope central heating source and The character of the spectrum of the source (be it - not much else matters! The character of the spectrum of the source (be young stars or an AGN) makes little difference since the primary photons at short wavelength are absorbed in the innermost boundary layer of the dust 列 envelope. The radius of this inner boundary is set by the radius at which the dust is heated to sublimation (see Section 8.2.2). The overall mass of dust of course determines the opacity and therefore the radius at which the dust of course determine, and thus the 'effective dust temperature' which is We will see below that the model SEDs of the FIR sources can be observed. Wingle parameter - the luminosity-to-mass ratio, $L / M$ characterised by a single parameter and thus the problem has, in essence, really just one independent variable as long as the source structure is simple (e.g., a single source with radial fall-off in density and no clumping). The compactness of the dust cloud is parametrised by a radial power law density distribution which can be varied but for the discussion below I adopt $R^{-1}$

Using the temperature profiles derived above, I have computed emergent spectra for a source of central luminosity $10^{12} L_{\odot}$ with overlying dust

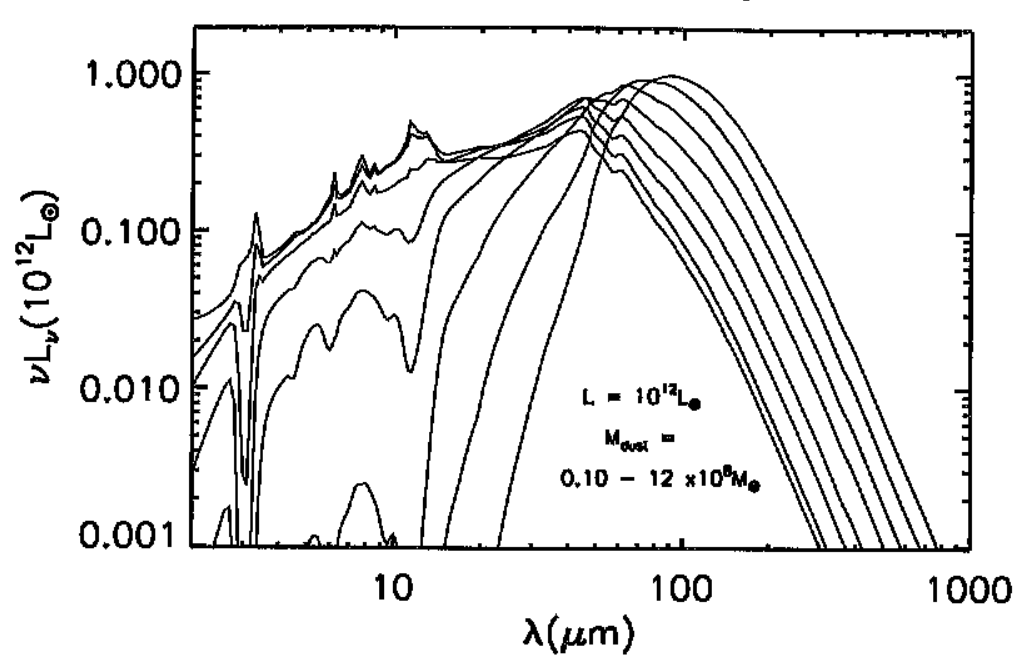

Fig. 8.13. The IR SEDs of dust cloud models with $10^{12} L_{\odot}$ are shown for increasing masses of overlying dust and an $r^{-1}$ density distribution. The dust heating is provided by both the central source and secondary photons from warm dust (see text). The peak shifts to longer wavelength as the dust opacity increases and the R-J tail rises linearly with dust mass.

masses ranging from $10^{7-9} M_{\odot}$ (i.e., total ISM masses $\sim 100$ times greater or. $\left.10^{9-11} M_{\odot}\right)$. These parameters are directed towards ULIRGs and SMGs but the results can easily be scaled to lower- or higher-luminosity objects. As mentioned above, the critical model characteristic is the luminosity-to-mass ratio. For this modelling, the dust distribution is taken to vary as $R^{-1}$ but similar results are found with other reasonable power laws. The inner radius is taken at $T_{\mathrm{d}}=1000 \mathrm{~K}$, but this is, of course, not critical since the hot dust is covered by the overlying colder dust unless the cloud is optically thin at short wavelengths. The outer radius was taken at $2 \mathrm{kpc}-$ this also is not critical since the dust is cold and optically thin to the secondary radiation at the largest radii.

Figure 8.13 shows the emergent specific luminosities $\left(\nu L_{\nu}\right)$ for the models with different enveloping dust masses but constant overall luminosity. Here one clearly sees the effect of varying dust mass and opacity. The clouds of lower dust mass have non-exponential short-wavelength SEDs due to the lack of high dust extinction on the short-wavelength side of the SED peak, and hence the hotter dust in the interior is exposed to our view. This contrasts with the higher-mass clouds which show a peak shifted to relatively longer wavelength - due to the fact that dust extinction short of the peak precludes photons escaping from the hotter interior radii. Figure 8.13 also 

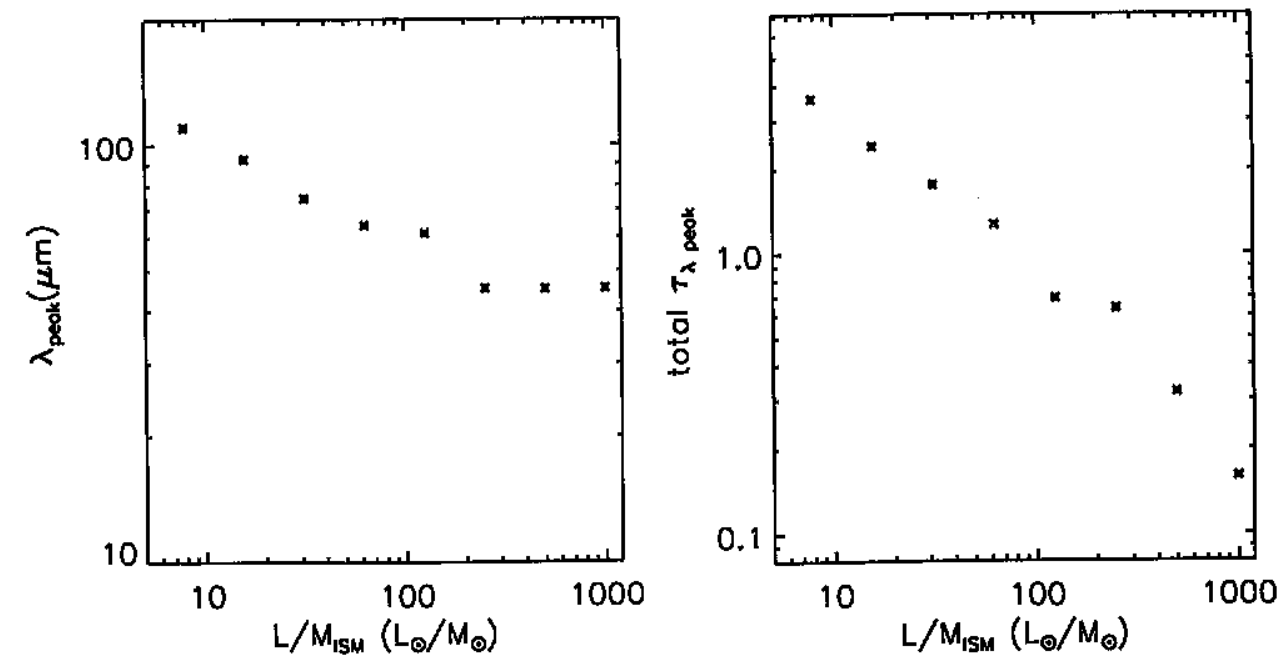

Fig. 8.14. The variation in the peak wavelength for $L_{\nu}$ and the optical depth through the cloud at the peak wavelength are shown for varying $L / M_{\text {ISM }}$ values with a density distribution of dust $\propto R^{-1}$. (Models run for power laws of $0,-1$ and -2 exhibit similar behaviour as long as the clouds are optically thick.)

clearly demonstrates the anticipated correlation (see Section 8.2.6) between the flux on the long-wavelength R-J tail and the dust mass.

To quantify some of the spectral characteristics, Fig. 8.14 shows the shift in the wavelength of peak emission ( $\lambda_{\text {peak }}$, left panel) and the optical depth at $\lambda_{\text {peak }}$ to the cloud surface (right panel) for the radial shell contributing most to the emergent luminosity. The right panel illustrates what was said earlier based on analytics - typically the opacity at the peak will be $\sim 1$ for a large range of overlying dust masses. Also, since the opacity must be $\sim 1$ at the peak, the wavelength of peak emission will be determined by both opacity and dust temperature in realistic models.

Figure 8.15 shows the radius and dust temperature at which the largest fraction of the overall luminosity escapes as a function of the luminosity-tomass ratio. The total luminosity is of course spread over a broad range of radii except in the most optically thick models (low $L / M_{\text {ISM }}$ values).

Figure 8.16 shows the ratio of total IR luminosity (at $\lambda=8-1000 \mu \mathrm{m}$ ) to the specific luminosity at $\lambda=850 \mu \mathrm{m}$ (i.e., a point on the power-law R-J tail) It should be obvious from this figure that there is really no good single value for the conversion factor from submm flux density $\left(\lambda_{\text {rest }} \gtrsim 150 \mu \mathrm{m}\right)$ to total IR luminosity - these models all had the same total luminosity but varying dust masses. Thus it is impossible to reliably estimate the total FIR luminosity from R-J flux measurements unless there are additional constraints,
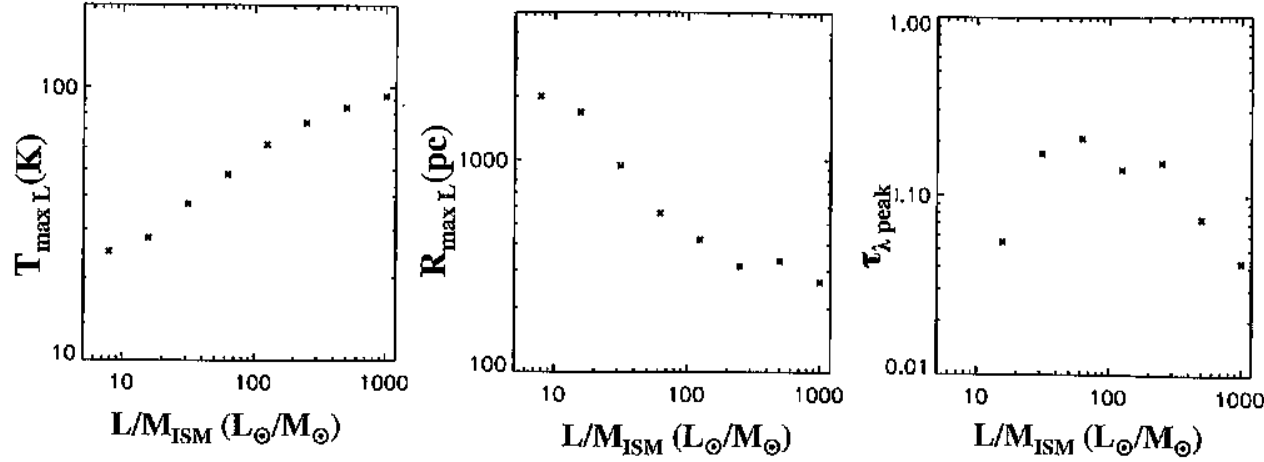

Fig. 8.15. Left and middle panels show the dust temperature and radius of the shell in the model producing the largest fraction of the overall luminosity for varying $L / M_{\text {ISM }}$ values. The right panel shows the optical depth at the peak wavelength from this shell to the outer cloud surface - illustrating the fact that the emergent
luminosity is produced at $\tau \lesssim 1$. In most cases, the output luminosity is in fact luminosity is produced at $\tau \lesssim 1$. In m

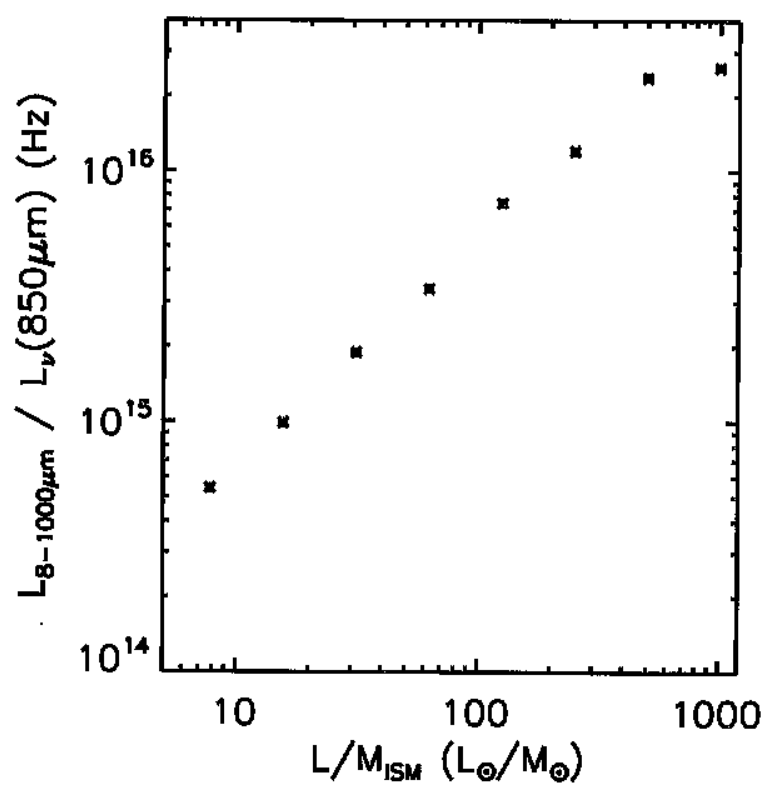

Fig. 8.16. The variation in the total IR luminosity to restframe $850 \mu \mathrm{m}$ flux is shown for a range of $L / M_{\text {ISM }}$ values, illustrating the impossibility of estimating the total IR luminosity from a single long-wavelength flux measurement.

for example on the luminosity-to-mass ratio or the ISM mass (e.g., from CO, or dynamical mass estimates). One simply must have measurements close to the IR SED peak. 


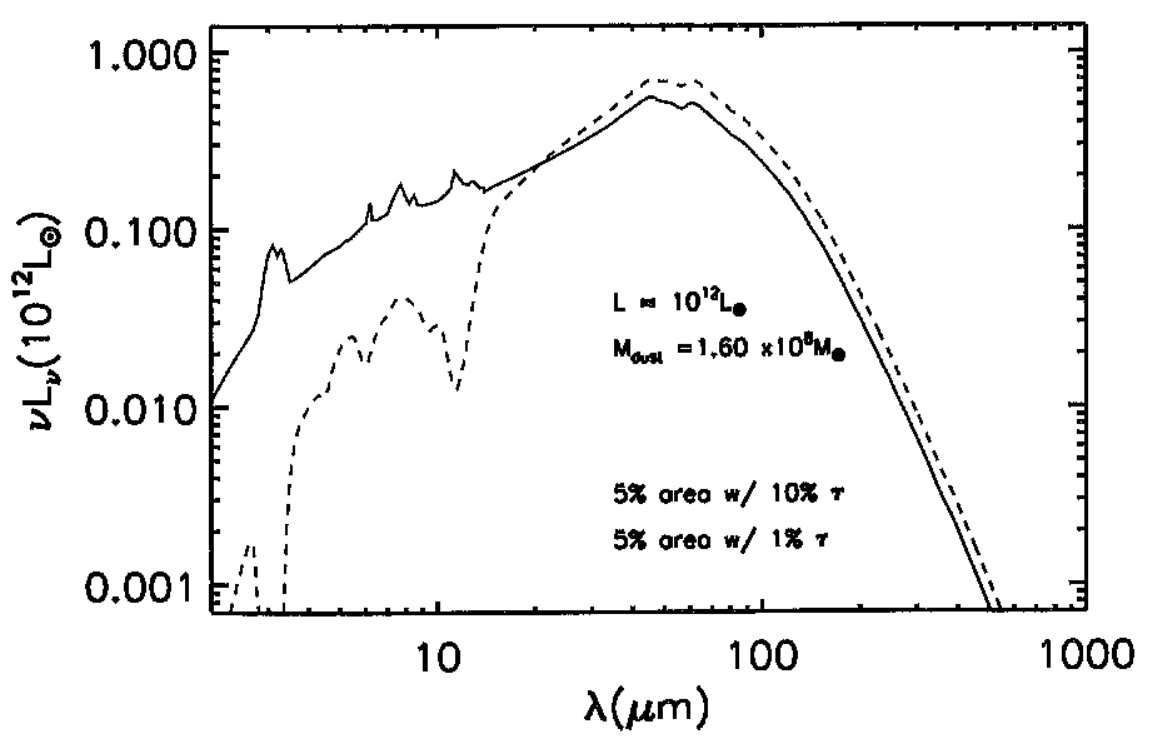

Fig. 8.17. Models with complete covering (dashed line) and with $5 \%$ of the area having extinction reduced to $10 \%$ and $1 \%$ of the complete covering model (solid line) are shown to illustrate that a slightly clumpy dust distribution is required to model the observed short-wavelength hot dust emission. Transient small grain heating is included by calculating the UV-visual radiation energy density at each radius and inserting the PAH emission following Draine \& Li (2001).

There are also some limitations or lessons from this modelling. Most noteworthy is the polycyclic aromatic hydrocarbon (PAH) emission which arises from transiently heated small dust grains. This small grain component was included by calculating the UV-visual radiation energy density at each radius and inserting the PAH emission following Draine \& Li (2001). These short-wavelength features require the emergence of flux from regions where the grains are hot. In sources showing such features, the dust obscuration must be clumpy, or multiple optically-thin and -thick sources must be contributing. To illustrate this possibility, Fig. 8.17 shows the effect of reducing the dust column in $5 \%$ of the surface area to a value $10 \%$ of the normal column density - the NIR/MIR becomes much stronger and the silicate feature appears in absorption.

The presence or absence of detectable PAH or silicates features depends on the covering fraction of the overlying optically-thick dust - their detection should not be taken as a reliable indicator of one mode of star formation (as done by Elbaz et al. 2011) or alteration of the grain abundances since their presence can depend simply on geometry and source non-uniformity.

\subsubsection{Summary}

We have developed a very simple model for the FIR emission which can be implemented for fast radiative transfer computations relevant to dustembedded luminosity sources. There are several important conclusions:

(a) For a central luminosity source, the dust temperature profile with radius can be modelled with very simply power laws: $T_{\text {dust }} \propto r^{-0.42}$ for optically thin dust and $T_{\text {dust }} \propto r^{-0.5}$ for optically thick dust. In a realistic source, it will be optically thin at the very innermost radius, optically thick at intermediate radii and optically thin at the outer radii; the temperature profile is then a piecewise fitting together of these two power laws.

(b) To model the FIR sources, it is vital to recognise that there are two entirely independent parameters: the total luminosity and the mass of dust (and to a much lesser extent the radial profile of the dust distribution). Since the temperature profile scales with $L$, then most sources may be uniquely characterised by the ratio $L / M_{\mathrm{dust}}$.

(c) Using these temperature profiles, it is then straightforward and quick to calculate the emergent SED in the IR as a function of the ratio $L / M_{\text {dust }}$.

(d) At the FIR peak, the emission is often optically thick (based on the steep fall-off in the SED to shorter wavelengths) but the optical depth will not be very large (i.e., $\tau_{\text {peak }} \sim 2-4$ ) since otherwise the radiant luminosity would not be able to escape.

(e) The effective dust temperature for the emergent luminosity can be determined approximately from the wavelength of the peak and then used to estimate an effective radius for the source.

(f) Flux measurements on the R-J tail of the SED are uniquely capable for determination of the total dust mass (with a factor of two reliability) since the dust is optically thin. And if one can assume a dust-to-gas abundance ratio, such measures lead to quick and reliable estimates of a distant galaxy's total ISM content.

(g) Determination of the total luminosity and hence the SFR requires observations near the peak at $\lambda_{\text {rest }} \sim 100 \mu \mathrm{m}$; it can not be reliably estimated using a single long wavelength R-J flux measurement (as is often done for SMGs).

(h) In optically-thick sources (as judged by the steep fall-off on the short side of the SED peak), the MIR enaission at $\lambda<20 \mu \mathrm{m}$ requires that the dust be somewhat clumped with incomplete covering in order to see the hot dust and the silicate and PAH features. 
8.3 Star formation in galaxies - two modes

\subsubsection{Quiescent or normal mode of star formation}

As discussed in Section 8.1, the star-forming GMCs are self-gravitating with internal velocity dispersions implying an effective internal turbulent pressure typically 100 times the external diffuse ISM pressures. One's physical intuition should then suggest that disturbances in the external, diffuse ISM will have little influence in general on the rate of formation of stars within GMCs. With this in mind, one might expect that normally SFRs will simply depend linearly on the overall mass of $\mathrm{H}_{2}$ and/or the internal density of the cloud and this logic should hold in other galaxies as long as the GMC properties are roughly similar. Galaxy type or the location within a galaxy should have little influence on what happens within the self-gravitating clouds.

Observations of $\mathrm{CO}$ emission (tracing the mass of GMCs) and star formation tracers (FIR luminosity or $\mathrm{H} \alpha$ ) have generally shown a linear proportionality between. SFR and $\mathrm{H}_{2}$ mass distributions. For example, the overall Galactic HII region radial distribution shown in Fig. 8.2 is similar to that of $\mathrm{H}_{2}$ as traced by $\mathrm{CO}$. On the scale of individual star-forming clouds, one might expect sizable fluctuations in the SFR per unit mass since the overall Galactic star formation efficiency (SFE) is low with a characteristic star formation timescale $\sim 10^{9} \mathrm{yr}$, i.e., much longer than the GMC internal dynamical timescale $\sim 10^{7} \mathrm{yr}$. In addition, the GMCs may have somewhat variable mean densities which of course alters their internal dynamical timescale. For a sample of individual Galactic GMCs (including ones with and without associated Hrr regions), Fig. 8.18 (left panel) shows an approximately 'linear' correlation between molecular gas mass $M_{\text {virial }}$ and $L_{\mathrm{IR}}$ or the SFR (Scoville \& Good 1989) for GMCs with masses $10^{4}$ to over $10^{6} M_{\odot}$. The scatter off this relation is a factor $\sim 4$. There is also a clear trend for the most massive clouds which all have Hil regions, presumably in the spiral arms, to have elevated ratios.

Given the tendency of the spiral arm GMCs to have somewhat elevated luminosity-to-mass ratios, it is probably wisest to use the mean Galactic luminosity-to-mass ratio $\left(4 L_{\odot} / M_{\odot}\right)$ to define a 'normal' mode of star formation. Such a ratio of $L_{\mathrm{IR}} / M_{\mathrm{H}_{2}} \simeq 4 L_{\odot} / M_{\odot}$ implies a characteristic star formation timescale $\sim 10^{9} \mathrm{yr}$ for conversion of molecular ISM into stars. The ULIRGs (at $L_{\mathrm{IR}}>10^{12} L_{\odot}$, most of which are gas-rich galaxy mergers) exhibit $a \sim 10-20$ times higher $L_{\mathrm{IR}} / M_{\mathrm{H}_{2}}$, hence a 10-20 times shorter timescale.

In summary, there is good basis (empirical from the observations and theoretical intuition from what we know regarding the GMC structures) for an
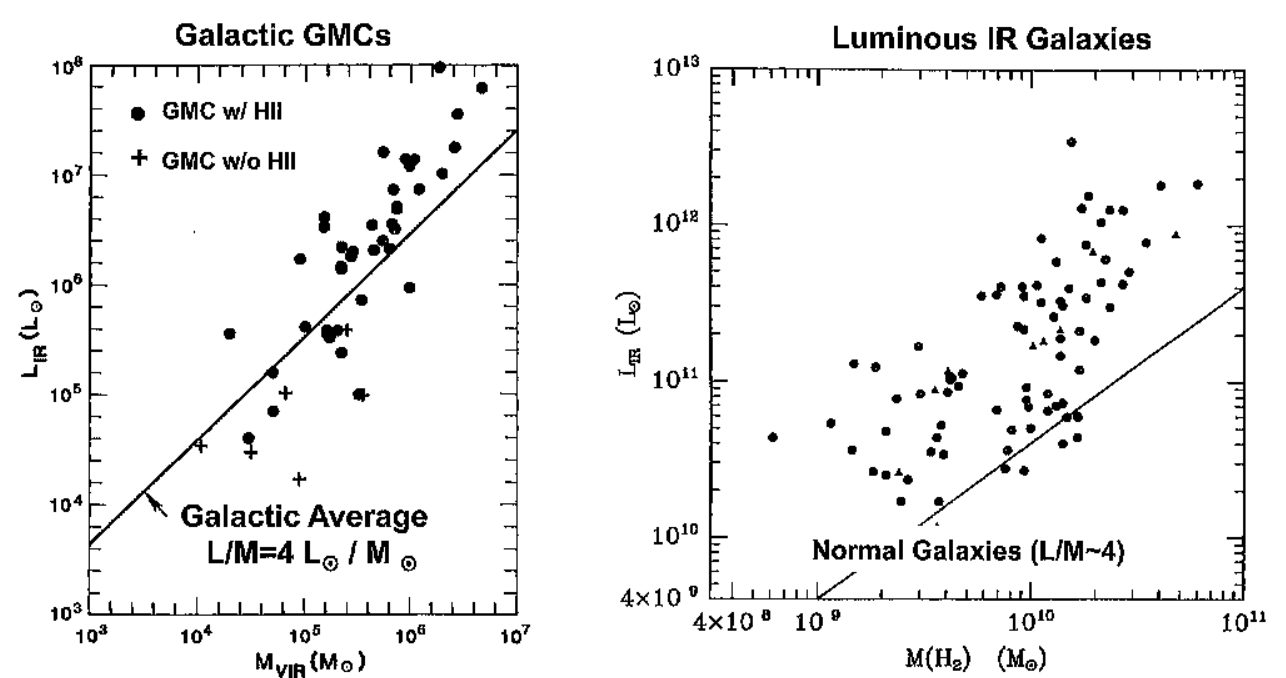

Fig. 8.18. Left panel: the relation between IR luminosity and $\mathrm{H}_{2}$ mass is shown for a large sample of Galactic GMCs with (dots) and without HII regions (crosses), compared with the overall Galactic average obtained from dividing the total Milky Way IR luminosity by the total $\mathrm{H}_{2}$ mass (Scoville \& Good 1989). M 51 and many
Wat Way IR luminosity by the total $\mathrm{H}_{2}$ mass (Scoville \& Good 1989). M 51. and many
other nearby spiral galaxies have mean ratios similar to that of the Milky Way. The right panel shows a similar plot for a local sample of IR bright galaxies (Sanders et al. 1991). The ULIRGs at $L_{\mathrm{IR}}>10^{12} L_{\odot}$ show much higher luminosity-to-mass ratios, indicating that they are converting gas to stars 10-20 times faster.

approximately linear mode of star formation. From local galaxies such as the Milky Way and M 51 , this is quantified at $\simeq 1 M_{\odot}$ per year per $10^{9} M_{\odot}$ of $\mathrm{H}_{2}$, or

$$
\mathrm{SFR}=\left(M_{\mathrm{H}_{2}} / M_{\odot}\right) \cdot 10^{-9} \quad M_{\odot} \mathrm{yr}^{-1}
$$

\subsubsection{Dynamically-driven starburst mode}

At the same time, there are clearly instances, such as those of the IR-bright galaxies, galaxy nuclei and the spirals arms and bars of normal galaxies, where the SFE is significantly enhanced to a level such that they can be classified as starbursts. Figure 8.18 (right panel) shows a plot of the IR luminosities and molecular gas masses for IR-luminous galaxies (LIRGs, Sanders et al. 1991) - all of them have considerably elevated luminosity-tomass ratios compared to the average ratios for the Milky Way and nearby spiral galaxies like M 51. Both the luminous IR galaxies and the individual spiral arm GMCs have exceptionally high rates of massive star formation (presumably they are also forming low-mass stars). In such regions, the SFE 
(based on the ratio of $L_{\mathrm{IR}} / M_{\mathrm{H}_{2}}$ ) may be enhanced by a factor ten compared to that in Equation 8.29.

In both the spiral arms and the ULIRGs, this elevated SFE appears correlated with non-circular galactic dynamics and high concentrations of dense gas. Galaxy merging leads to dissipative deposition of gas to the centres of the merging systems and spiral arm streaming motions cause crowding of the GMC galactic orbits. As a result of the non-circular motions and concentration of gas clouds, cloud-cloud collisions will be more frequent. If such collisions occur at relative velocity greater than the GMC internal velocities (few $\mathrm{km} / \mathrm{s}$ ), the collisions can compress the internal gas mass and significantly elevate the SFE (Scoville \& Hersh 1979; Tan 2000). This high-SFE mode might therefore be referred to as a dynamically-driven starburst where the SFE is 10-50 times that given in Equation 8.29 - occurring in galaxy mergers or localised regions such as spiral arms and nuclear bars of normal galaxy disks.

\subsubsection{Star formation 'laws'}

Over the years a number of prescriptions have been proposed to describe the rates of star formation within galaxies. Early on, Scoville \& Good (1989) and Young \& Scoville (1991) advocated that the SFR varied linearly with the mass of molecular gas - based on observations of Galactic GMCs and the similarity of molecular gas radial distributions in nearby, normal galaxies to the radial distributions of SFR tracers (e.g., blue light, H $\alpha$ and FIR). Exthe radial distributions of SFR tracers (e.g., blue light, Ha and $\alpha$ in the analysis then led to the well-known Kennicutt-Schmidt SFR law with SFR $\propto \Sigma_{\text {HI }+\mathrm{H}_{2}}^{1.4}$ and a threshold cutoff surface density below which the SFR decreased more steeply. In my opinion, the atomic gas (HI) exhibits almost no correlation with the SFR tracers (recently shown clearly by Bigiel et al. 2008 and Schruba et al. 2011) and is not directly relevant to the star formation process in the inner parts of spiral galaxies. (The non-integral, $\sim 1.4$, dependence of the SER in the Kennicutt-Schmidt star formation law is likely due to including $\mathrm{HI}$ in the analysis and is without a direct physical link to star formation except via the formation of molecular clouds and due to the inclusion of starburst galaxy nuclei. As emphasised above, the GMCs are long-lived and stars form from them at low efficiency, so the fact that the $\mathrm{HI}$ is needed to form molecules does not justify a physical connection to the star formation process.)

The most recent compilations have been done by Bigiel et al. (2011) and Krumholz et al. (2012). The former advocate a simple linear dependence on molecular gas surface density; the latter propose a 'volumetric' law $\dot{\Sigma}_{*}=f_{\mathrm{H}_{2}} \epsilon_{\mathrm{ff}} \Sigma / \tau_{\mathrm{ff}}$ where the factors entering are the $\mathrm{H}_{2}$ mass fraction, the
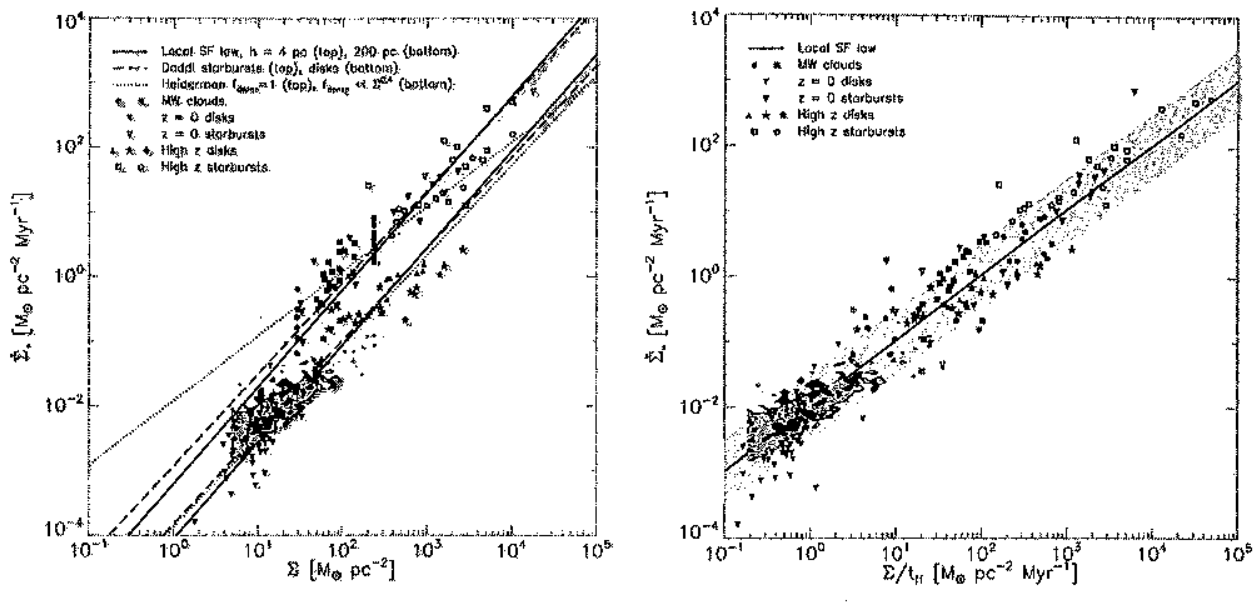

Fig. 8.19. Left panel shows the compilation of observational determinations of the SFR and total gas surface densities by Krumholz et al. (2012) together with severa proposed SFR prescriptions. The right panel shows the alignment of these data improved when the gas surface densities are normalised by a free-fall timescale, i.e. a scalelength, to provide a fixed 'volumetric' law.

efficiency of star formation per free-fall time, the total gas surface density and the free-fall time. Their compilation of observational data is shown in Fig. 8.19, together with the various star formation laws they evaluated. Although their proposed volumetric law provides a good fit to a large range of data, the evaluation of $\tau_{\mathrm{ff}}$ requires that a scaleheight for the gas be assumed and this is not easily derived from the observations so the quality of fit is largely dependent on what is assumed.

At this point, I believe the best approach is simply: 1) assume star formation occurs only in the molecular gas (i.e., do not include atomic gas in the analysis since it is not physically relevant) and 2) assume two modes of star formation: a) a quiescent or normal star formation mode which depends linearly on the mass or surface density of $\mathrm{H}_{2}$; and b) a dynamically-driven mode (relevant to mergers, spiral arms and bars) which is also linear with $\Sigma_{\mathrm{H}_{2}}$ but has a rate constant up to 20 times that of the first mode.

Clearly, for distant galaxies it would be nice to have a more precise star formation law with dependence on detailed properties of the clouds (such as their internal density and the details of the local galactic velocity field streaming and disparsion) but this will not be observationally accessible for many many years. Let's keep the star formation prescriptions close to the relevant observational capabilities! 


\subsubsection{Distinguishing normal star formation and starbursts:} concentration and timescale.

How does one define a starbursting system (as opposed to a normal starforming galaxy) and what are the observational discriminants between these two modes? I think the most physically meaningful definition for a starburst is a galaxy or area within a galaxy where the rate of conversion of existing ISM into stars is such that the ISM will be consumed in a time significantly less than the typical $M_{\mathrm{ISM}} / \mathrm{SFR} \sim 1$ Gyr timescale for local galaxies like the Milky Way. This SFE can be observationally characterised by the simple ratio $L_{\mathrm{IR}} / M_{\mathrm{H}_{2}}$.

Alternatively, one might use the specific SFR per unit stellar mass (SSFR $=\mathrm{SFR} / M_{*}$ ) to identify galaxies where the characteristic timescale (1/SSFR) is much shorter than the 'likely' age of the galaxy (which is obviously less than the age of the Universe at the galaxy's redshift). At redshift $z=1.5-2.5$, Rodighiero et al. (2011) argue that there is a tight 'main sequence' of star-forming galaxies with fairly constant SSFR at all stellar masses (see Fig. 8.29); they then identify starburst objects with SSFR significantly above this main sequence. Unfortunately, the SSFRs found for the main sequence at $z \sim 2$ are higher by a factor $\sim 15$ than those at low redshift so the discriminant between the two star formation modes is evolving in time - without a clear physical basis, be it a higher gas content, smailer stellar mass at early epochs or an increasing efficiency/rate in converting existing gas to stars. And, of course, if the definition of the main sequence is evolving in time, the fraction of galaxies found to be 'bursting' will depend entirely on how far off the main sequence a galaxy must be to be so classified. If most galaxies at high redshift were, in fact, bursting, then the 'main-sequence' SSFR would be elevated as observed and the dispersion would simply reflect the range of burst activity. Clearly, it would be most important now to analyse the morphologies of the so-called main-sequence galaxies to classify the merging/interacting versus undisturbed percentages.

Definitions for starburst classification which compare the SSFR to the galaxy age or better, using the timescale for exhausting the ISM are more physically based than simply using the dispersion about the main sequence. In the near future we can anticipate that ALMA will be measuring gas contents for large samples of high- $z$ galaxies.

Another approach which might be used to identify starburst activity is the luminosity density or concentration of the activity. In starbursting systems, one expects that a large fraction of the luminosity from the young stars will be absorbed by dust and emerge in the FIR and that the luminosity energy
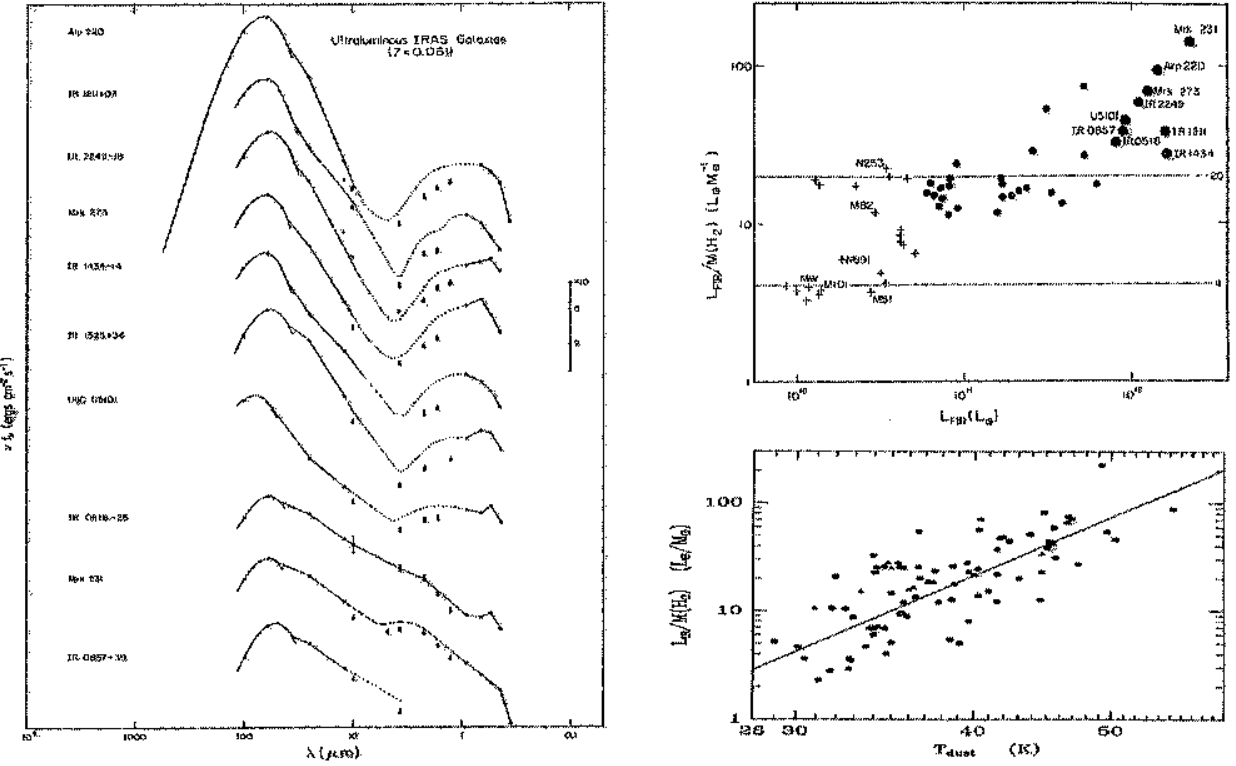

Fig. 8.20. The SEDs, $L_{\mathrm{IR}} / M_{\mathrm{H}_{2}}$ mass ratios and fitted dust temperatures are shown for the complete sample of 20 ULIRGs at $z<0.1$ from Sanders et al. (1988) and Sanders et al. (1991)

density within the active region will be high. As discussed in Section 8.2.9, good IR SED sampling allows one to classify the observed SED according to the ratio $L_{\mathrm{IR}} / M_{\text {dust }}$ which is proportional to $L_{\mathrm{IR}} / M_{\mathrm{ISM}}$. Sources with high luminosity density (either AGN or intense starbursts) may also have high 'apparent' colour temperatures, providing they are not completely enshrouded by dust which is optically thick out to $100 \mu \mathrm{m}$. One can reasonably estimate a characteristic source size using Equation 8.13 and a luminosity density from $L_{\mathrm{IR}} /\left(4 \pi R_{\text {thick }}^{3} / 3\right)$.

\subsubsection{Starbursts in ULIRGs}

In Fig. 8.20 the SEDs, $L_{\mathrm{IR}} / M_{\mathrm{H}_{2}}$ mass ratios and fitted dust temperatures are shown for the complete sample of 20 ULIRGs at $z<0.1$ from Sanders et al. (1988) and Sanders et al. (1991). The SEDs peak at $\lambda \gtrsim 120 \mu \mathrm{m}$ with dust colour temperatures $30-50 \mathrm{~K}$ and the FIR-to- $\mathrm{H}_{2}$ mass ratios are 30-200 $L_{\odot} / M_{\odot}$. As noted above, these ratios are a factor 10-20 higher than the luminosity-to-mass ratios in local spiral galaxies, indicating a 10-20 times shorter timescale for converting ISM to young stars. 


\subsubsection{Arp 220 - a prototypical ULIRG}

In the spirit of 'one example can provide more understanding than many generalisations', I think it is very worthwhile to look in detail at current observations of Arp 220. Of course not all ULIRGs are the same as Arp 220, but one can readily see the essence of the starburst mode in this object.

$\operatorname{Arp} 220$, at $77 \mathrm{Mpc}$, is one of the nearest and the best-known ultraluminous merging system $\left(L_{8-1000 \mu \mathrm{m}}=1.5 \times 10^{12} L_{\odot}\right)$. To power the IR output, star formation must be occurring at a rate of $\sim 10^{2} M_{\odot} \mathrm{yr}^{-1}$. Visual wavelength images reveal two faint tidal tails, indicating a recent tidal interaction (Joseph \& Wright 1985), and high-resolution ground-based radio and NIR imaging show a double nucleus (Baan \& Haschick 1995; Graham et al. 1990). The radio nuclei are separated by $0.98 \mathrm{arcsec}$ (Baan \& Haschick 1995), corresponding to $350 \mathrm{pc}$. Millimetre-wave imaging provides a unique capability to probe such starburst nuclei in dusty LIRG/ULIRGs since the dust is optically thin at long wavelengths. Virtually all of the ULIRGs observed so far have massive concentrations of molecular gas in the central few kiloparsec (Scoville et al. 1997; Downes \& Solomon 1998; Bryant \& Scoville 1999; Tacconi et al. 1999).

Arp 220 has been imaged at high resolution in the $2.6 \mathrm{~mm}$ CO line (Scoville et al. 1991), $3 \mathrm{~mm} \mathrm{HCN}$ (Radford et al. 1991), $1.3 \mathrm{~mm}$ CO (Scoville et al. 1997; Downes \& Solomon 1998; Tacconi et al. 1999) and most recently in the $0.9 \mathrm{~mm} \mathrm{CO}$ and $\mathrm{HCO}^{+}$lines (Sakamoto et al. 2009). The $\mathrm{CO}(2-1)$ line emission exhibits two peaks separated by 0.9 arcsec, and a larger inclined disk of molecular gas (Scoville et al. 1997; Downes \& Solomon 1998; Sakamoto et al. 1999; Tacconi et al. 1999). At 0.5 arcsec resolution (Sakamoto et al. 1999), the $\mathrm{CO}$ and $1.3 \mathrm{~mm}$ continuum imaging shown in Fig. 8.21 reveals counterrotating disks of gas in each of the IR nuclei. The kinematic data require very high mass concentrations in both of the nuclei. The nuclear disks are counter-rotating, consistent with the notion that the most complete and violent merging should be associated with counter-rotating precursor galaxies in which there is naturally angular momentum cancellation during the merging. The masses of each nucleus are apparently dominated by the molecular gas - a common finding of the ULIRG galaxy studies (Bryant \& Scoville 1999) The nucleus of Arp 220 has a total molecular gas mass $M_{\mathrm{H}_{2}} \sim 9 \times 10^{9} M_{\odot}$ within 300 pc (Scoville et al. 1997; Downes \& Solomon 1998; Sakamoto et al. 1999; Tacconi et al. 1999). This $\mathrm{H}_{2}$ mass within $300 \mathrm{pc}$ is $3-4$ times that of the entire Milky Way (most of which is in the ring at $4-8 \mathrm{kpc}$ ).

Although Arp 220 has enormous masses of molecular gas, one should not visualise this gas being contained in self-gravitating GMCs like those in
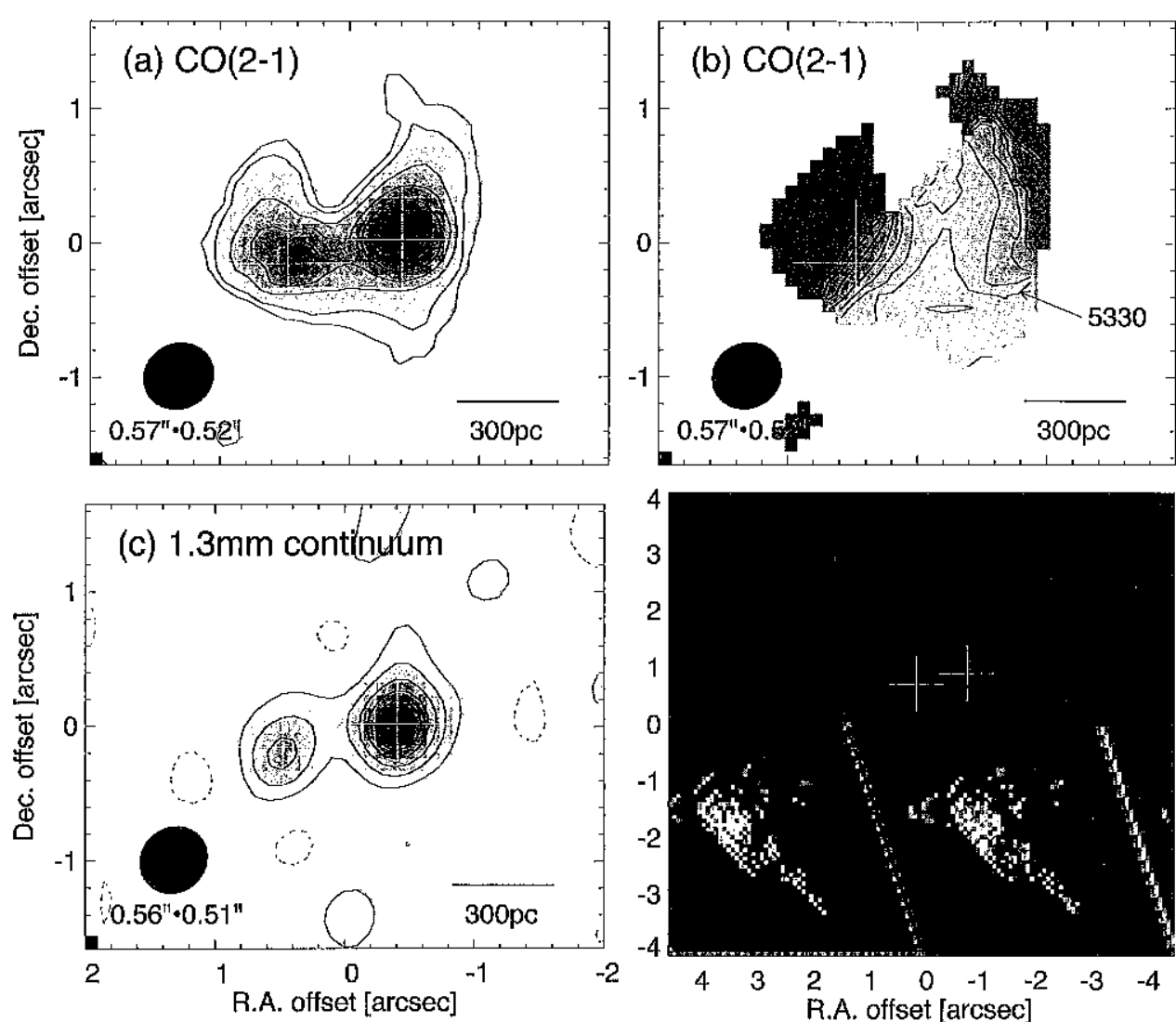

Fig. 8.21. The merging galactic nuclei of Arp 220 are shown in 0.5 arcsec resolution imaging of the $\mathrm{CO}(2-1)$ and dust continuum emission (Sakamoto et al. 1999). The four panels show: a) continuum-subtracted $\mathrm{CO}(2-1)$, b) the $\mathrm{CO}$ mean velocities, c) tour panels show: a) continuum-subtracted $1.3 \mathrm{~mm}$ dust continuum, and $\mathrm{d}$ ) the total $\mathrm{CO}$ emission including the extended

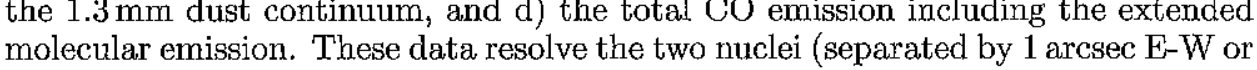
$350 \mathrm{pc}$ ) and the crosses indicate the $1.3 \mathrm{~mm}$ dust continuum peaks. In the molecular gas emission, the two galaxy nuclei have counter-rotating disks as seen in the upperright image.

the Galaxy. The high brightness temperature of the observed CO emission (Scoville et al. 1997) requires that the gas distribution be continuous, i.e., filled nuclear disks, not a cloudy medium. The mass density within the disks implies a visual extinction $A_{V} \sim 2000$ mag perpendicular to the disks! Clearly, studying such regions can only be superficial in the optical/NIR and must rely on much longer-wavelength observations.

The western nucleus in Arp 220 exhibits hard X-ray emission (Iwasawa et al. 2005) and very high-velocity $\mathrm{CO}$ emission. The inner dust emission source seen at $\lambda \sim 1$ is extremely compact with $10^{9} M_{\odot}$ within $R<35 \mathrm{pc}$ 
(Downes \& Eckart 2007). Downes \& Eckart argue that the inferred dust temperature of $\sim 175 \mathrm{~K}$ implies a higher radiation energy density for its heating than could be provided by a compact starburst and therefore that this nucleus may harbour a luminous and massive black hole.

\subsubsection{An aside: $S g r A^{*}$ - an extraordinary ISM}

Given the very limited spatial resolution in observations of distant nuclei such as Arp 220, it is instructive and cautionary to look at the properties of the ISM in our own Galactic nucleus despite the fact that it is not currently very active. The massive black hole associated with the radio source Sgr $\mathrm{A}^{*}$ has a mass of $4 \times 10^{6} M_{\odot}$ - derived from the motions of stars within the central 1 pc (Genzel 2006; Ghez 2007). A circumnuclear disk (CND) at radius out to $\sim 3 \mathrm{pc}$ is seen with both ionised gas and dense molecular clumps on the exterior. Figure 8.22 shows the ionised gas as probed in the $\mathrm{HI} \mathrm{P} \alpha$ line at $1.87 \mu \mathrm{m}$ (greyscale; Scoville et al. 2003) in a structure termed the mini-spiral and the dense molecular gas as probed in the $3 \mathrm{~mm} \mathrm{HCN}$ line (contours; Christopher et al. 2005). The molecular gas is in a clumpy ring at $1-3$ pc radius. The ionised gas in the mini-spiral may be infalling or outflowing; the ionised gas at the edge of the disk in the southeastern arm probably arises from photoionisation at the inner edge of the molecular cloud ring.

The total mass of the molecular gas within $3 \mathrm{pc}$ of the black hole is variously estimated at $1-5 \times 10^{5} M_{\odot}$ and has an orbital time $\sim 10^{5} \mathrm{yr}$. Given that the black hole is only $\sim 10$ times more massive it is clear that very little of the nearby ISM will actually make it to the black hole, unless we are viewing an extremely atypical epoch for Sgr $\mathrm{A}^{*}$. In any case, it is certainly the case that the ISM here is not being accreted on an orbital timescale since the luminosity of $\operatorname{Sgr~}^{*}$ is $\ll 10^{6} L_{\odot}$.

Just as was the case for Arp 220, our intuition about the properties of the ISM in the disk of our galaxy (i.e., self-gravitating GMCs) clearly cannot be transferred directly to the ISM in the Milky Way nucleus. The molecular gas clumps in the CND have extraordinary properties! Their sizes are $<1 \mathrm{pc}$ but densities $10^{7-8} \mathrm{~cm}^{-3}$ (Christopher et al. 2005). (By contrast, a typical Galactic $10^{5} M_{\odot}$ GMC has a diameter $40 \mathrm{pc}$ and mean density $\sim 300 \mathrm{~cm}^{-3}$, see Section 8.1.3). The higher densities are required near $\operatorname{Sgr} \mathrm{A}^{*}$ if the clumps are to be stable against tidal disruption, given their proximity to the central point mass. If the dust-to-gas ratio in the clumps is similar to the standard Galactic value, then the inferred column densities of $10^{25} \mathrm{~cm}^{-2}$ imply an extinction of $A_{V} \sim 10^{4} \mathrm{mag}$ through each clump. Since the molecular ring/torus is clearly not appearing edge-on, its angular momentum is

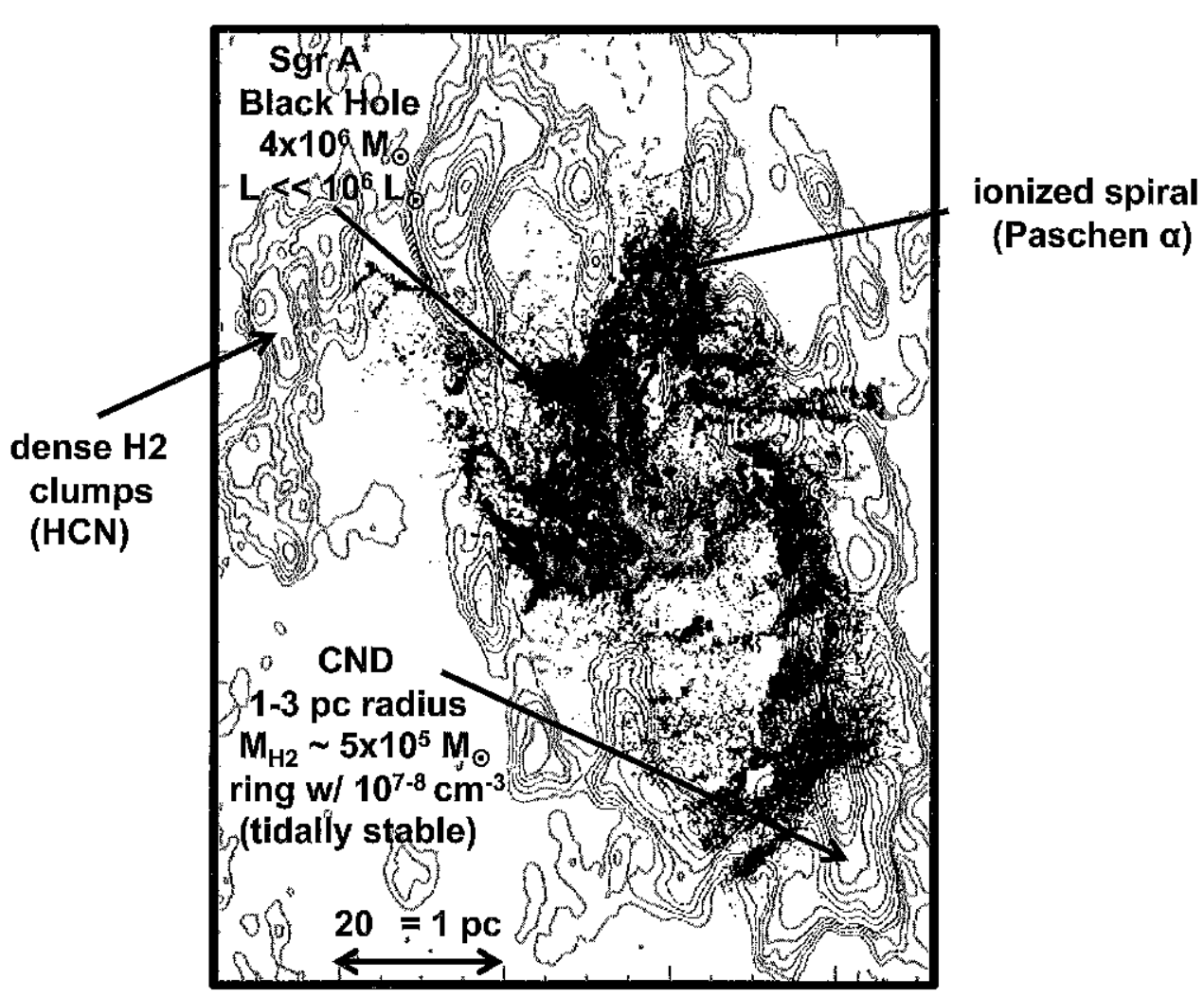

Fig. 8.22. The circumnuclear disk (CND) in the Galactic nucleus disk in ionised gas (greyscale) as imaged in the $\mathrm{Hr} \mathrm{P} \alpha$ line at $1.87 \mu \mathrm{m}$ (Scoville et al. 2003) and the dense molecular gas (contours) seen in HCN emission (Christopher et al. 2005). The ionised gas is distributed in a 'spiral' pattern and at the inner surfaces of the neutral molecular clumps. The densities in the molecular gas are $\sim 10^{7-8} \mathrm{~cm}^{-3}(!)$, orbiting at $1-3$ pc radius.

not perpendicular to the Galactic plane. The ring therefore probably resulted from the accretion and disruption of a single cloud at non-zero height out of the Galactic plane, rather than smooth accretion over time from the larger nuclear disk. If such a cloud arrived at the point where it was tidally disrupted with a non-zero height the resulting fragments might end up as clumps in an orbital plane inclined to the Galactic plane, as is observed for the CND.

At present there is no evidence of star formation within the molecular clumps despite their very high densities and extraordinarily short internal dynamical timescales $\sim 10^{4} \mathrm{yr}$. However, the very high extinctions suggested above would preclude detection of embedded young stars and the only means of detecting them might be via radio detection of ultra-compact HII regions 

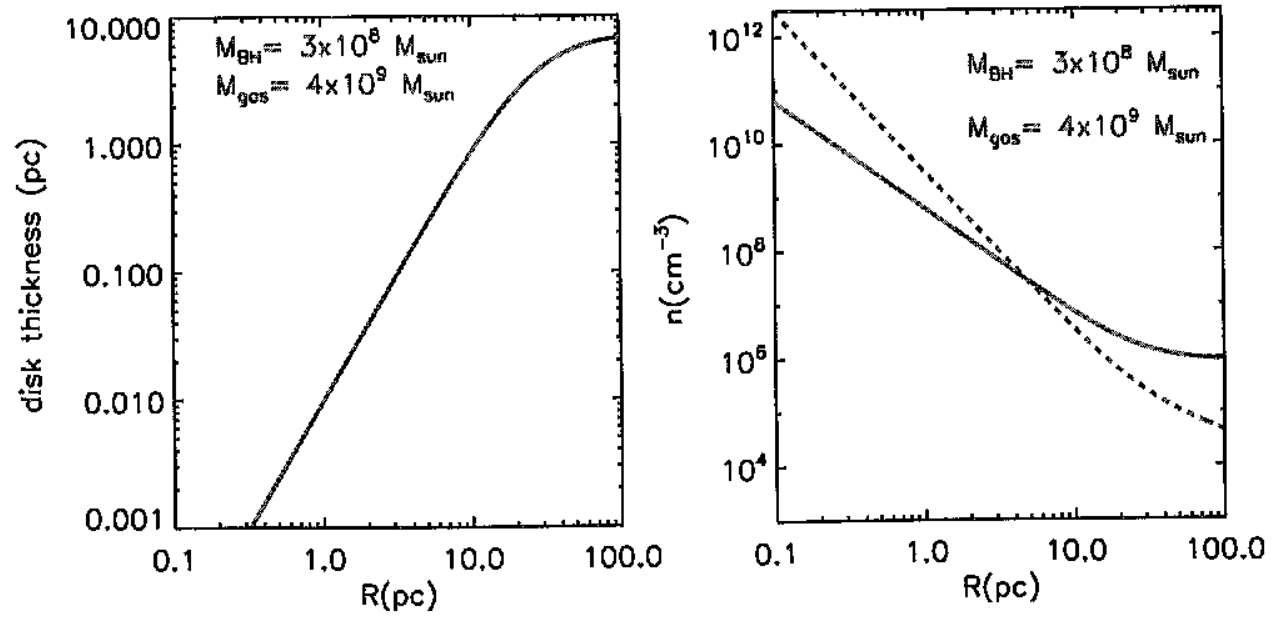

Fig. 8.23. A very crude model for the disk in gas-rich merging systems like Arp 220 is shown. We assume a central point mass (black hole) and a uniform gas surface density disk extending to $100 \mathrm{pc}$ radius. The turbulent velocity dispersion within the disk is taken to be $50 \mathrm{~km} / \mathrm{s}$ (based on Arp 220; Scoville et al. 1997). The disk thickness as a function of radius for hydrostatic equilibrium is shown in the right panel and the mean gas density in the left panel (solid red line). The critical density required for stability of a self-gravitating object (e.g., a cloud) in the disk is shown by the denities expected for the very simple assumptions and underscores the fact that self-gravitating clouds should not form in the inner disk due to tidal disruption.

or maser emission. The unusually high densities of the molecular clumps must reflect the fact that at lower density they would not be tidally stable and therefore could not exist for long (see Section 8.3.8).

\subsubsection{Nuclear starburst disks}

As a first step toward understanding the massive gas disks like that seen in Arp 220, we consider an extremely simple model with uniform gas surface density out to radius $100 \mathrm{pc}$. At the centre of the disk, we assume a point mass of $4 \times 10^{8} M_{\odot}$ - either a central black hole or a nuclear star cluster. The disk is assumed to be self-gravitating and in hydrostatic equilibrium with a sound speed of $50 \mathrm{~km} / \mathrm{s}$, representative of the turbulent velocity dispersion measured in Arp 220. Although this is meant only as an illustrative, gedanken, experiment, it clearly shows that the conditions are inescapably different from those of star-forming clouds in the Galactic disk.

In Fig. 8.23, the disk thickness and mean gas densities are shown as a function of radius out to $100 \mathrm{pc}$. The thickness of the disk is typically less
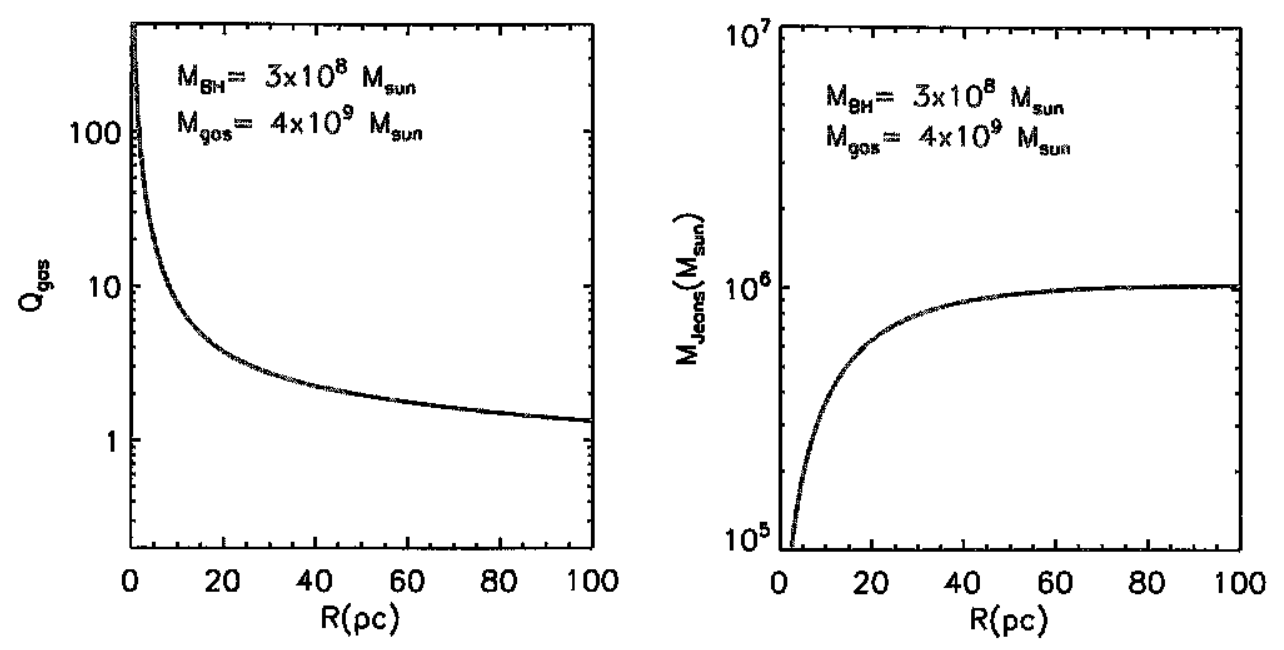

Fig. 8.24. The left panel shows the Toomre $Q$ stability parameter $(Q<1$ unstable) for the disk parameters in the previous figure and the right panel shows the Jeans mass for collapse/self-gravitation assuming a sound speed of $50 \mathrm{~km} / \mathrm{s}$ and neglecting tidal shear.

than $10 \mathrm{pc}$ and the mean densities are everywhere $>10^{6} \mathrm{~cm}^{-3}$, getting up to $10^{10} \mathrm{~cm}^{-3}$ near the centre. The right panel of Fig. 8.23 also shows the gas density required for tidal stability (dashed line) - clearly demonstrating that it will be difficult to form stable clouds within the central $10 \mathrm{pc}$ radius. Within this region, we should expect a more continuous gaseous disk structure, not a cloudy ISM as found in the centre of our Galaxy.

The gravitational stability of the disk can also be seen from the Toomre $Q$ parameter shown in Fig. 8.24 (left panel). Within the inner $10 \mathrm{pc}, Q \gg 1$ and it is very hard to form self-gravitating structures in the ISM, such as clouds. On the other hand, outside $10 \mathrm{pc}$ radius such clouds might form (if they are not disrupted by cloud collisions) but their masses are required to be very large, exceeding $10^{6} M_{\odot}$.

At the inner boundary of the nuclear disk $(R \sim 1-5 \mathrm{pc})$, one expects that the dust will sublimate and any ISM inside this radius will be dust-free and therefore have much lower radiative opacity, permitting radial accretion inwards. On the other hand, at the sublimation boundary, there will be a strong radiation pressure gradient in the outward direction, tending to push the inner edge of the dust accretion disk to larger radii. The physics of this transition zone should indeed be interesting - the material pushed outwards will intercept orbit disk gas with higher specific angular momentum, and the dust sublimation interface will also be Rayleigh-Taylor unstable, 
perhaps leading to radial streams of accretion flow. I leave these details to the students to work out!

\subsubsection{Maximum-rate starbursts - the dust Eddington limit}

For Arp 220, the estimated nuclear SFR of $100-200 M_{\odot}$ per year is at least a factor of ten less than the maximal rate, obtained by dividing the ISM by the orbital timescale of $\sim 2 \times 10^{6} \mathrm{yr}$. One very effective means of restricting the star fromation (or even ejecting the dense ISM) is via the radiation pressure on dust of the central starburst (or AGN) luminosity. For a self-gravitating gas and dust mass, the effective 'Eddington' limit is approximately $500 L_{\odot} / M_{\odot}$, similar to the overall mass-to-light ratio measured in the Arp 220 nucleus $\left(10^{12} L_{\odot} / 3 \times 10^{9} M_{\odot}\right)$. For higher luminosity-to-mass ratios, the ISM is blown out of the region.

The significant role of radiation pressure in high-mass star formation and starbursts for feedback through radiation pressure on buildup has only recently been appreciated. The outward radiation pressure in a starbursting cloud core or galactic nucleus will dominate self-gravity at radius $R$ when

$$
\frac{L\langle\kappa\rangle}{4 \pi R^{2} c}>\frac{G M_{\mathrm{R}}}{R^{2}}
$$

where $\langle\kappa\rangle$ is the effective radiative absorption coefficient per unit mass. Although the original stellar radiation is primarily UV and visible, the dust in the cloud core absorbs these photons and re-radiates the luminosity in the IR. The 'effective' absorption coefficient takes account of the fact that outside the radius where $A_{V} \sim 1 \mathrm{mag}$, the luminosity is at longer wavelengths where the dust has a reduced absorption efficiency. For the standard ISM dust-to-gas ratio (Draine \& Li 2007), $A_{V}=1$ mag corresponds to a column density $\mathrm{N}_{\mathrm{H}}=2 \times 10^{21} \mathrm{~cm}^{-2}$ and

$$
\langle\kappa\rangle=312 \frac{\lambda_{V}}{\lambda_{\text {eff }}(R)} \quad \mathrm{cm}^{2} \mathrm{~g}^{-1}
$$

where $\lambda_{\mathrm{eff}}(R)$ is the absorption coefficient-weighted mean wavelength of the radiation field at radius $R$ and for simplicity, we have adopted a $\lambda^{-1}$ wavelength dependence for the absorption efficiency.

Combining Equations 8.30 and 8.31, we find that the radiation pressure will exceed the gravity when

$$
(L / M)_{\mathrm{cl}} \geq 42 \frac{\lambda_{\mathrm{eff}}}{\lambda_{V}} \frac{L_{\odot}}{M_{\odot}} .
$$

For $\lambda_{\text {eff }} \sim 3-10 \mu \mathrm{m}, \lambda_{\text {eff }} / \lambda_{V}$ is approximately 10 and the radiation pressure limit is $\sim 500 L_{\odot} / M_{\odot}$. Thus, for luminosity-to-mass ratios exceeding this value radiation pressure will halt further accretion. For a forming OB star cluster, this luminosity-to-mass ratio is reached at about the point where the upper main sequence is first fully populated, i.e., a cluster with approximately $2000 M_{\odot}$ distributed between 1 and $120 M_{\odot}$. A similar 'Eddington' limit applies for any dust-embedded starburst region (see Scoville et al. 2001; Scoville 2003; Murray et al. 2005; Thompson et al. 2005). It turns out that much larger-scale nuclear starbursts such as that in Arp 220 have approximately reached the same empirical limit of $500 L_{\odot} / M_{\odot}$, suggesting that nuclear starburst activity may also be regulated by a balance of self-gravity and radiation pressure support.

\subsubsection{AGN - starburst: observational connections}

Evidence has also accumulated for an evolutionary link between merging ULIRGs and UV/optical quasi-stellar objects (QSOs) as suggested by Sanders et al. (1988): similar local space densities for ULIRGs and QSOs; continuity of FIR SEDs smoothly transitioning between the two classes; the occurrence of AGN-like emission lines (Veilleux et al. 1999) and significant point-like nuclei (less than 0.2 arcsec - Scoville et al. 2000) in $30-40 \%$ of the ULIRGs; and the association of both ULIRGs and some QSOs (MacKenty \& Stockton 1984; Bahcall et al. 1997) with galactic interactions (see the review article by Sanders \& Mirabel 1996). Whether the entire QSO population had precursor ULIRGs (implying that galactic merging is the predominant formation mechanism for AGN) is certainly not yet settled and at lower AGN luminosities, much of the activity is probably not directly associated with galaxy merging or interactions.

Two possible scenarios linking the ULIRG and luminous AGN phenomena are: 1) that the abundant ISM, deposited in galactic nuclei by merging, fuels both the nuclear starburst and feeds the central black hole accretion disk; or alternatively, 2) the post-starburst stellar population evolves rapidly with a high rate of mass return to the ISM in the galactic nucleus - leading to sustained fuelling of the black hole (e.g., Norman \& Scoville 1988).

\subsubsection{AGN - starburst: theoretical connections}

The largest potential sources of fuel for AGN are the nuclear ISM (until it has been cleared out) and the mass loss associated with normal stellar evolution of stars in the galactic nucleus. Most recent discussion has assumed the former link; so I would like to briefly discuss the latter possibility here 
- partially as a stimulus for further investigation by students. For a nuclear starburst population, approximately $20 \%$ of the initial stellar mass will be lost via red giant mass-loss winds within the first $2 \times 10^{8}$ years (Norman $\&$ Scoville 1988). (The mass return associated with supernovae is probably significantly less.) Scoville \& Norman (1988) and Scoville \& Norman (1995) examined the fate of this mass-loss material - specifically to account for both the broad emission lines (BELs) and the broad absorption lines (BALs) seen in AGN. Since the stars will not be disrupted by AGN feedback once the nucleus becomes active, the stars can provide a long-term supply of fuel to power the AGN.

The physics of the dust shed by the stars in their stellar winds, particularly its evaporation, is critical to determining whether the mass-loss material accretes inwards to an accretion disk or is blown outwards by radiation pressure. This consideration leads naturally to a division of the central cluster environment into: 1$)$ an inner zone $(r \leq 1 \mathrm{pc})$ where the dust is heated to above the sublimation temperature and the lower opacity of the dust-free gas allows it to fall inwards, and 2) an outer zone ( $r \geq 1 \mathrm{pc})$ where the dust (and gas) survives and is driven outwards at velocities up to $0.1 c$ by radiation pressure.

It is interesting to note that the density required for tidal stability of a BEL cloud at $1 \mathrm{pc}$ radius from the black hole requires that the clouds must have extremely high internal densities. At 1 pc from a $10^{9} M_{\odot}$ black hole, the Roche limit density is $3 \times 10^{11} \mathrm{~cm}^{-3}$. This provides a strong argument for the stellar (rather than interstellar) scenario to account for the BELs, using the stellar masses to bind the emission line gas.

\subsection{Evolution of galaxies at high redshift}

Over the last 15 years extensive surveys of high-redshift galaxy evolution (e.g., GOODS, UDF, COSMOS, AEGIS and CANDELS) with space- and ground-based telescopes have dramatically increased our understanding of galaxy evolution in the early universe. All of these go very deep (typically $>27-30 \mathrm{mag}$ AB) but cover very different size areas - most also have excellent multi-wavelength, ancillary data. The largest survey (COSMOS) has over a million galaxies with photometry and photometric redshifts at $z=0.2-6$ and the deepest (UDF) now has detections at $z=6-8$, probing the first $1 \mathrm{Gyr}$ of cosmic time. Major evolution is seen in the galaxy properties: stellar mass, luminosity, size and SFR.

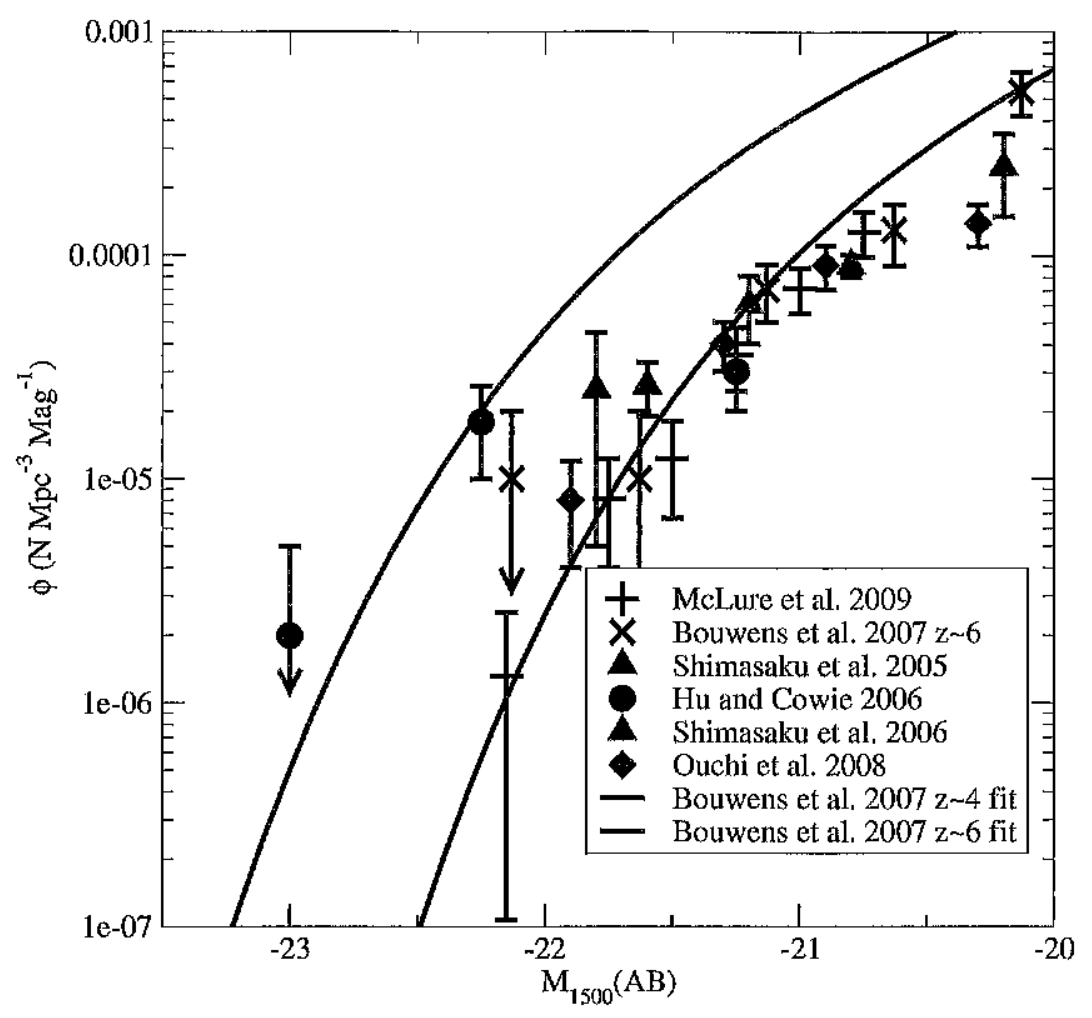

Fig. 8.25. A compilation of $z=4$ and 6 UV LFs for galaxies (Capak et al. 2011). The curves are Schechter function fits by Bouwens et al. (2007) for $z=4$ (blue) and 6 (black) galaxies and the points are derived for Lyman alpha emitters (LAEs) and Lyman break galaxies (LBGs).

\subsubsection{Luminosity and Mass Functions}

Figure 8.25 shows a compilation of recent determinations of the $z=4-6$ luminosity functions (LFs; Capak et al. 2011). Evolution of the UV LF (probing the distribution of star-forming galaxies) is clearly seen with the number densities increasing at all luminosities as one goes to lower redshift and there is apparent steepening of the low- $L$ power law going to higher redshift, i.e., more low-luminosity galaxies contributing. If this persists to higher redshift then it can be argued that the reionisation of the universe at $z \sim 10$ must have been produced by relatively low-luminosity galaxies (Robertson et al. 2010). At $z>6$, the samples are very small, $\sim 20-50$ objects with virtually no spectroscopic confirmation. Even at $z=6$, one can see large scatter in the different determinations of the LF, shown as points in Fig. 8.25. It is noteworthy that the high- $L$ portion of the LF is very poorly 


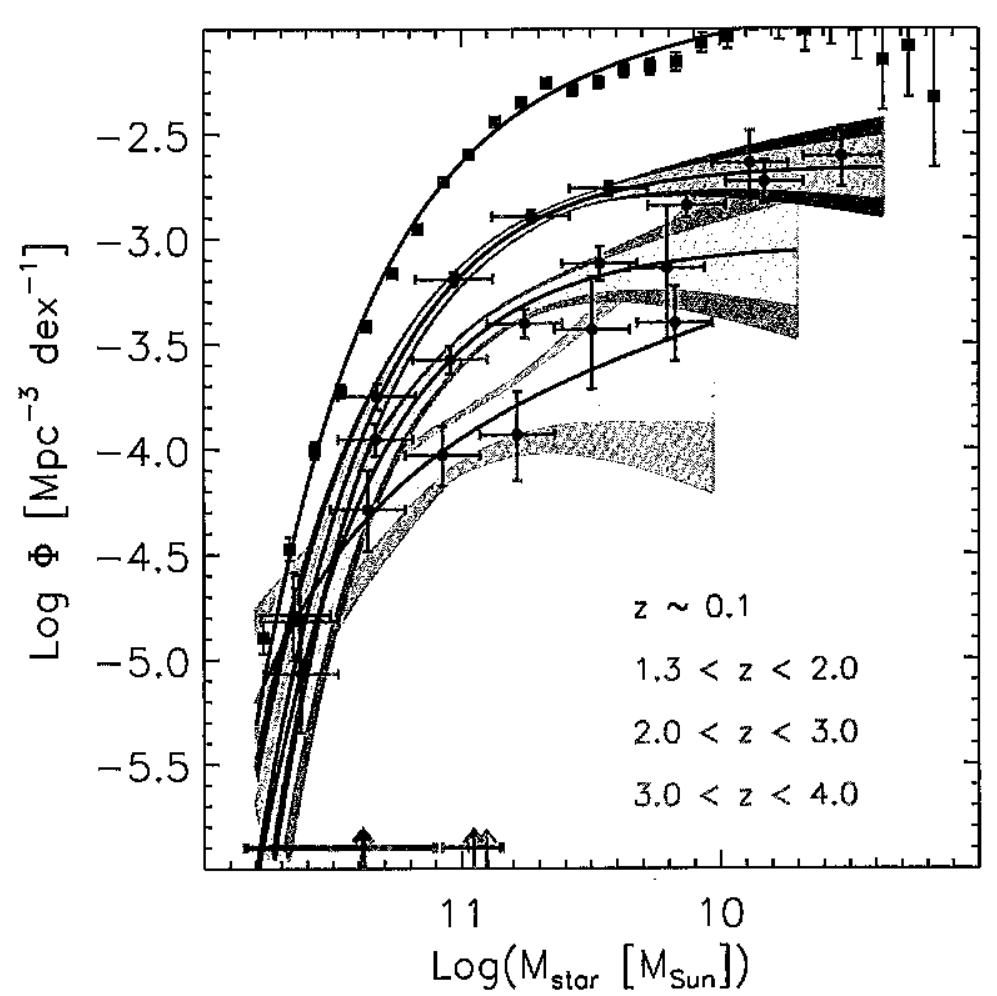

Fig. 8.26. The stellar mass function for galaxies at $z=0.1-4$ from Marchesini et al. (2009).

constrained (see Fig. 8.25) and could even be a power law rather than the Schechter function (exponential fall-off) used in fitting.

At lower redshifts, comparatively good samples exist for the stellar mass functions of galaxies (see Fig. 8.26). Strong growth in the number density of galaxies at all masses is seen but the largest increase at late epochs (low $z$ ) occurs in the lower-mass galaxies (see, e.g., Ilbert et al. 2010). This 'downsizing' of evolution at later epochs is also seen in the galaxies with star formation and AGN activity.

In principle, the buildup of stellar mass in galaxies as a function of time should be consistent with the evolution of star formation activity within galaxies in each mass bin. Discrepancies between these two might indicate the rate of galactic merging (assuming the masses and SFRs are reliable). This has been explored by Drory \& Alvarez (2008) who find reasonable consistency between the stellar mass buildup and the SFR evolution - providing the SFRs drop first in the highest-mass galaxies and galaxies typically undergo $\sim 1$ major merger after $z=1.5$ (see also Reddy 2011).
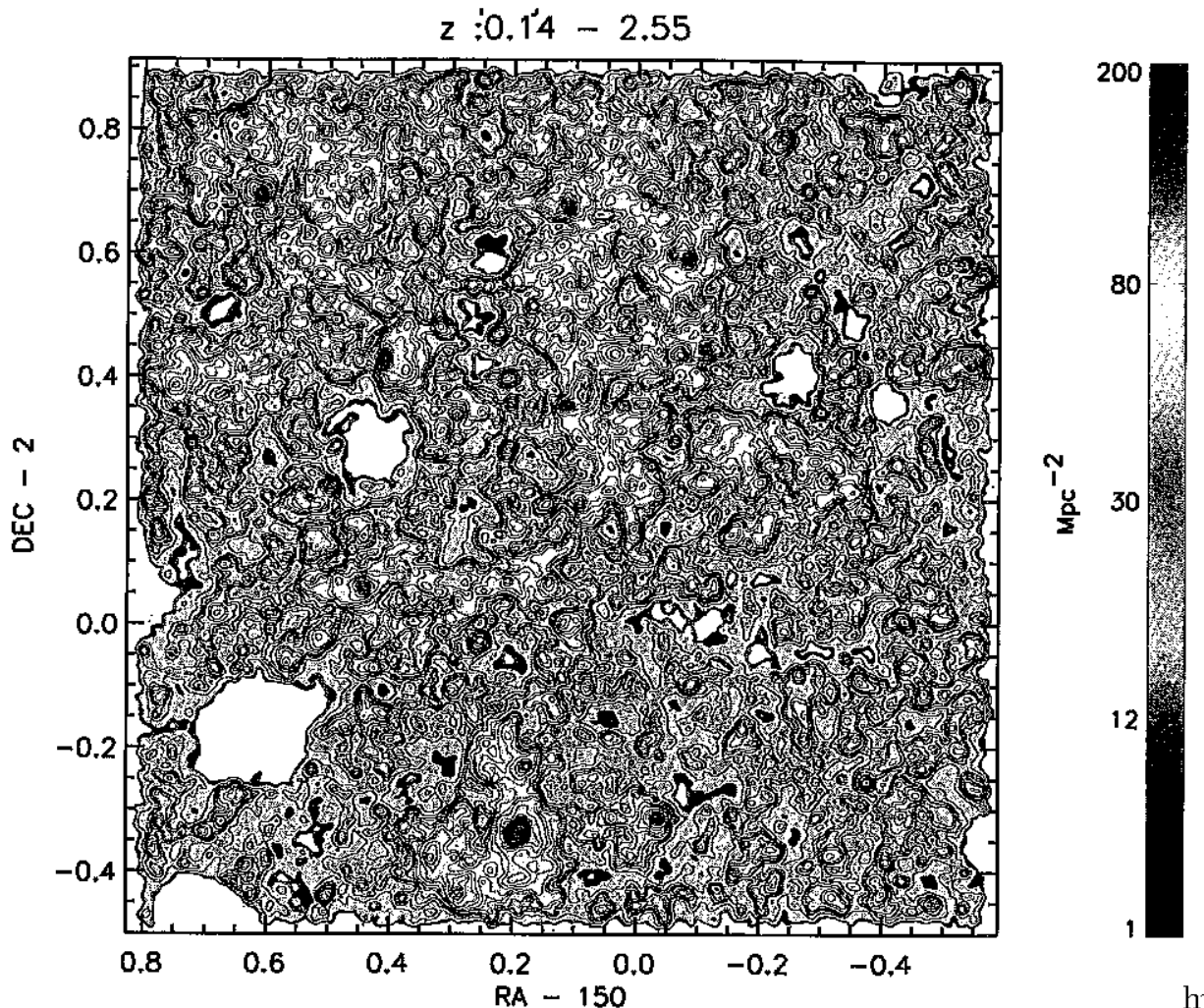

ht

Fig. 8.27. Large-scale structures mapped by the projected density of galaxies in redshift slices $(\Delta z=0.02-0.2)$ in the COSMOS survey field (Scoville et al. 2007a; Scoville et al. 2012). Over 200 significantly overdense regions are seen over the redshift range $z=0.1-2.55$.

\subsubsection{Environmental correlations}

In $\Lambda$ CDM simulations for the early Universe, it is the most massive, highly biased, structures which form earliest. Such structures will become the locations of massive galaxy clusters with the most massive galaxies built there first. The COSMOS survey (Scoville et al. 2007b) was specifically designed to probe a sufficiently large area on the sky (2 square degrees corresponding to $\gtrsim 50-100$ comoving $\mathrm{Mpc}$ at $z>0.5$ ) that the full range of environmental densities could be sampled at all redshifts. This enables both the mapping of the large-scale structure and the investigation of the correlation of galaxy evolution with environment. High-accuracy photometric redshifts and a large sample of spectroscopic redshifts enable the separation of galaxy structures along the line of sight. Figure 8.27 shows the 200 overdense regions of galaxies seen in COSMOS at $z=0.1-2.55$ (Scoville et al. 2012). 

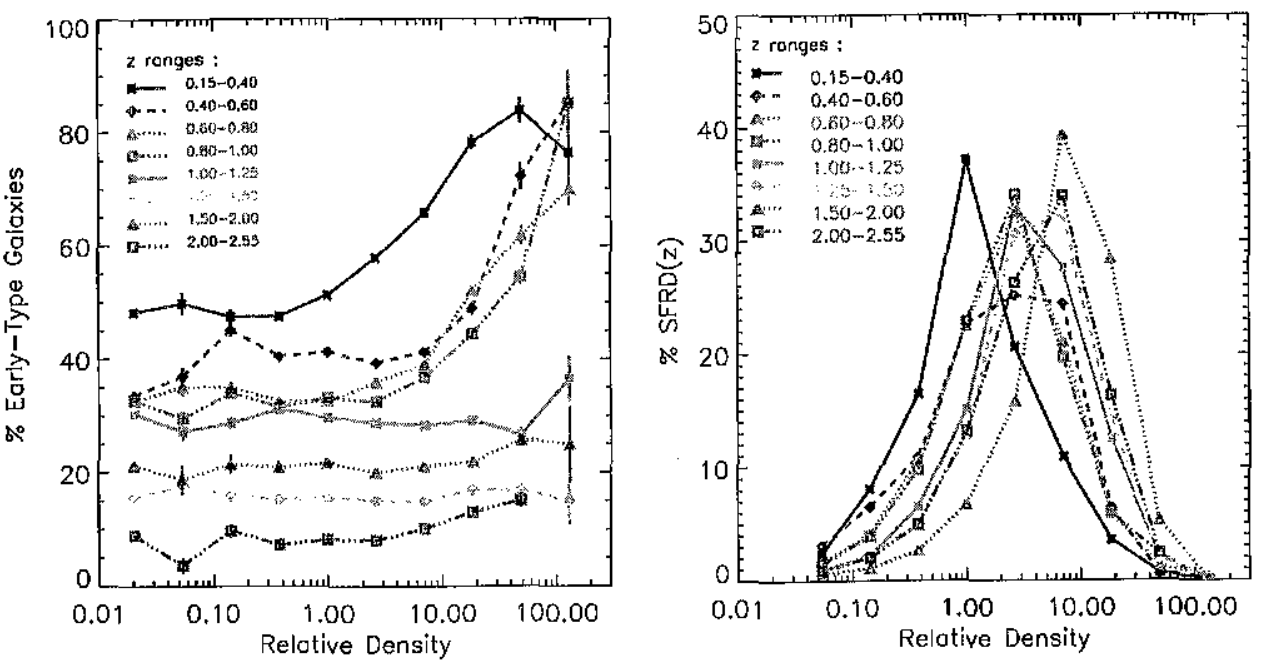

Fig. 8.28 Left panel: the early-type ( $\mathrm{E}-\mathrm{Sa}$ ) galaxy fraction is clearly correlated Fig. 8.28. Left panel: the early-type (E-Sa) galaxy fraction Fig. 8.27 (Scoville ct al. with both redshift and large-scale structure density from Fig. 8.27 (Scoville et al. density environments. Right panel: the percentage of the overall SFRD is shown as a function of redshift and large-scale structure density. The dominant environments for star formation shift to lower density at later epochs (Scoville et al. 2012).

Using the environmental densities shown in Fig. 8.27, I show the percentage of early-type galaxies (with SED corresponding to E-Sa galaxies) as a function of density and redshift in Fig. 8.28 (left panel). As is well known from many studies, the early-type galaxy fraction increases systematically to a fraction $\sim 50 \%$ at $z=0$. However, Fig. 8.28 (left panel) also clearly isolates the differential evolution associated with large-scale structure density - the early-type galaxies form first in the highest-density regions of the large-scale structure,

The evolution of the SFR density (SFRD $=\mathrm{SFR}$ per comoving volume) rises a factor 20 from $z=0$ to a peak at $z=2-3$. An important question is: in which environments is the star formation occurring at each epoch? Figure 8.28 (right panel) shows that the SFRD systematically shifts from dense to less dense regions of the large-scale structure as time progresses. Peng et al. (2010), using both COSMOS and SDSS data, show that the quenching of star formation activity in galaxies has two separable terms: one dependent on galaxy mass and the other on environmental density.

Recently, Rodighiero et al. (2011) have attempted to quantify the fraction of star formation occurring in starbursts versus normal-rate star formation at $z=1.5-2.5$. They make use of a $B Z K$ colour-selected sample of

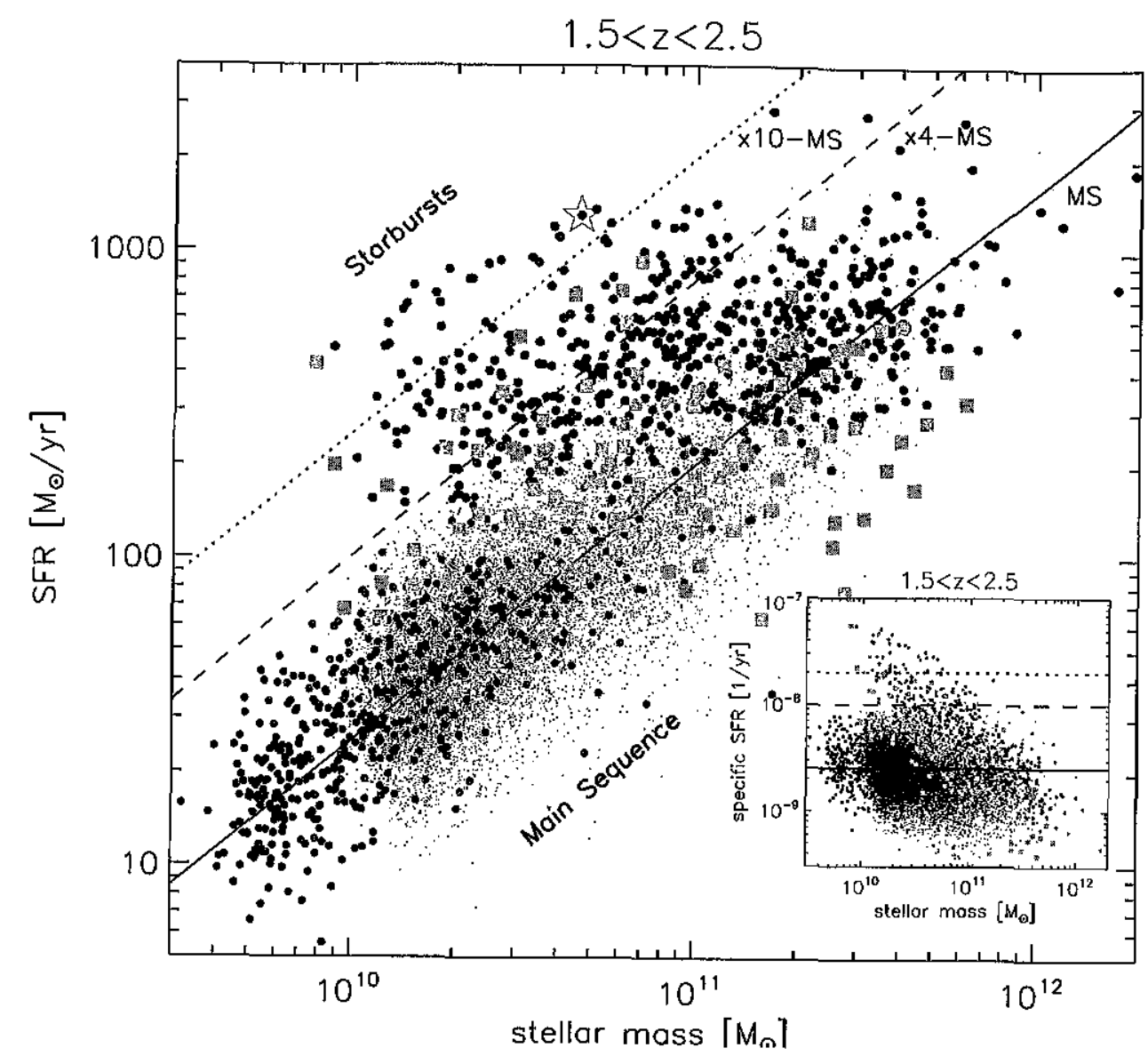

Fig. 8.29. The SFRs of three samples of galaxies at $z=1.5-2.5$ and stellar masses are shown (Rodighiero et al. 2011). The samples include $B Z K$-selected galaxies (black dots), GOODS (cyan) data and COSMOS (red) galaxies with Hershel PACS FIR detection. They use the $B Z K$ galaxies to define a 'main sequence' of starforming galaxies at this epoch and then classify the outlier (mostly IR-detected) galaxies at $>4$ times the SSFR on the main sequence as starburst-mode galaxies.

galaxies with low SFR and samples from GOODS and COSMOS, probing with Herschel and Spitzer the moderate- and high-FIR luminosity galaxies (see Fig. 8.29). The $B Z K$ galaxies are used to define a 'main sequence' of star-forming galaxies at this epoch. The outliers above the main sequence (mostly IR-detected) with more than four times the SSFR are then taken to be galaxies undergoing burst-mode star formation. Rodighiero et al. (2011) suggest that only $2 \%$ of the galaxies by number are undergoing starbursts and this population contributes $\sim 10 \%$ of the total SFRD at this epoch. This is an interesting approach to the issue, but it should be clear that the 
adopted definition of the main sequence and its spread will entirely determine the starburst percentages. In addition, some of the galaxies within the main sequence could well be merger-starbursts of originally lower-SSFR galaxies. As noted earlier, it would be advantageous to have a physicallybased definition of bursting rather than simply picking outliers as bursts. To take an extreme example, the latter definition would clearly be wrong if essentially a large fraction of the population were, in fact, bursting.

\subsection{Modelling star formation at high redshift:} same modes but different frequency

It is now well established that the activity associated with both star formation and AGN increases dramatically $(20 \times)$ out to a peak at $z \sim 2-3$ using SFRs determined by the UV continuum, FIR and radio continuum and that beyond $z=3$ there may be a gradual decline in the SFR density, although the measurements become increasingly difficult at these redshifts.

At high redshift, one might expect that the same two modes pertain, yet their relative importance could be quite altered. On the one hand, highredshift galaxies should have higher gas mass fractions than the typical $5-10 \%$ of low-redshift spiral galaxies like the Milky Way - leading to higher rates of star formation associated with the quiescent linear mode of star formation. But at the same time, one expects a greatly elevated rate of galactic merging/interaction, increasing the frequency of the starburst mode. Which of these dominates is not at all clear without a numerical simulation to track their relative importance, keeping track of the rare and brief mergers.

\subsubsection{Cosmic evolution:}

\section{$\mathrm{M}_{*}$ and $\mathrm{M}_{\mathrm{T}}$ and star formation luminosities}

In order to test the framework developed above against the observed cosmic evolution of galaxies, I developed a simple Monte Carlo simulation including: star formation in the quiescent and merger-driven burst modes, merging of dark matter haloes and their contained galaxies, and accretion of fresh gas from the external large-scale structure environment. The simulation starts at $z=6$ with a population of 10 million dark matter haloes having a mass function approximating that seen in simulations for $z=6$ (e.g., Heitmann et al. 2010). Each halo is started at $z=6$ with a galaxy of baryonic mass equal to the dark matter mass times the universal baryon fraction, with $90 \%$ of the baryons being gaseous ISM and $10 \%$ stellar mass. I then let the galaxy and dark matter halo population evolve with $50 \mathrm{Myr}$ timesteps down to $z=1$. At each timestep, gas is converted to stellar mass at the quiescent SFR given by Equation 8.29. At each timestep, haloes are also randomly sampled for having undergone a merger with a probam bility weighted as $(1+z)^{2.5} M_{\text {halo }}^{0.125}$ (Fakhouri \& Ma 2010). This merger rate is normalised such that $2 \%$ of the haloes merge per $50 \mathrm{Myr}$ at $z=6$. The secondary galaxy for each merger was also selected randomly from the galaxy population weighted by (mass ratio) ${ }^{-2.1}$ (Fakhouri \& Ma 2010). For those galaxies selected to merge, the SFE was increased by a factor of 10-50 (but only for one timestep). Gas accretion to the galaxy halo was taken as $M=6.6\left(M_{\text {halo }} / 10^{12}\right)^{1.15}(1+z)^{2.25} \times 0.165$ (Dekel et al. 2009 ) for $M_{\text {halo }}<10^{12} M_{\odot}$. For larger-mass haloes, we assumed simply that the accretion was cut off - either by the accretion shocks or by AGN feedback the former is a departure from Dekel et al. but some reduction of accretion is required in order to have the massive objects become gas-poor ellipticals at modest redshifts as shown observationally.

Minor non-critical details which were included were that: 1) the effective accumulation of stellar mass was taken to be $70 \%$ of the integrated star formation (i.e., assume $30 \%$ of the stellar mass is recycled eventually in mass loss), 2) the accretion of external gas to star-forming galaxy was delayed by 1 Gyr after it accreted to the halo boundary (to account for the infall time) and 3) during starbursts, ISM mass was shed from the galaxy at a rate equal to the SFR (only for the burst mode). The star formation luminosity (motivated to model the IR luminosity function) was taken very crudely as the total luminosity from stars formed in the last $50 \mathrm{Myr}$ plus that from young stars in earlier timesteps reduced by a factor of two in each timestep. Specifically, the luminosity associated with recent star formation was taken to be $10^{10} L_{\odot}$ per $M_{\odot}$ per year of star formation, based on observations of local galaxies.

\subsubsection{Need ISM replenishment by accretion}

This simplistic model was remarkably useful to explore critical aspects of the evolutionary scheme involving quiescent and burst-mode star formation with a reasonable gas accretion hypothesis. Figure 8.30 shows the evolved mass functions of stars and ISM gas and the star formation luminosity at $z=2.5$ and 1. For this figure, the simulation included merging galaxies and their associated starbursts but was without accretion of gas from the environment. In this case, the ISM runs down at a rate given by local Universe star formation laws and the original gas content of the haloes is exhausted by $z=2.5$ to a level much less than that seen either at $z=2$ or in present-epoch galaxies. 
mergers included $w / S B$ but no gas accretion
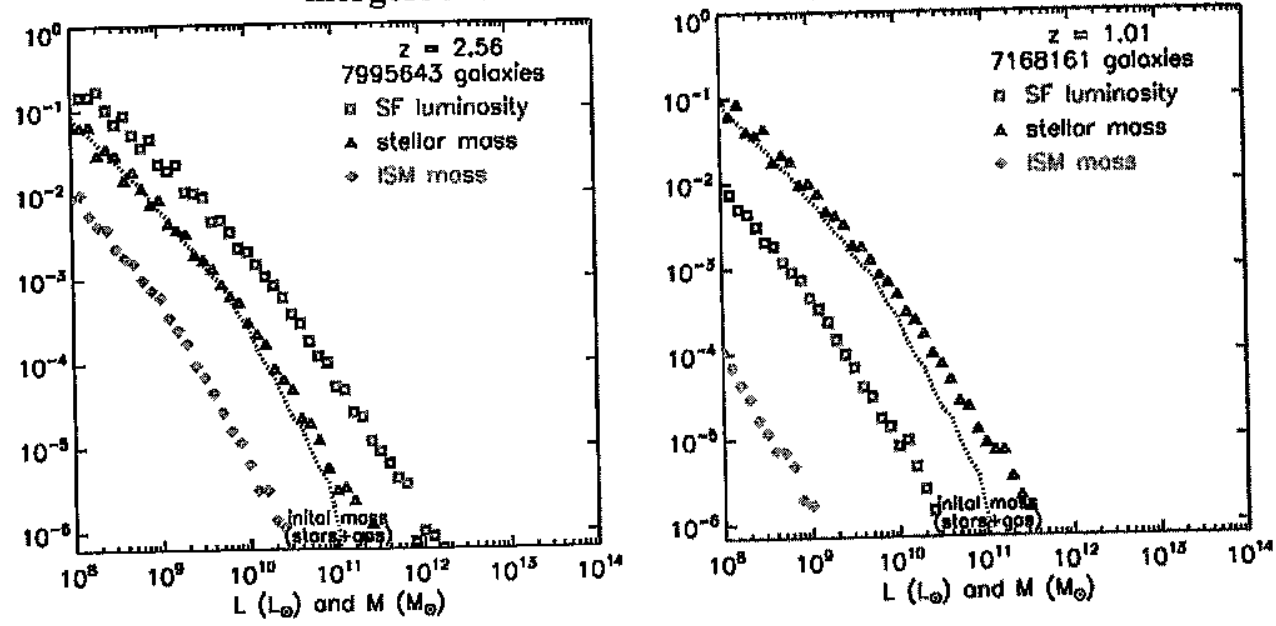

Fig. 8.30. The mass and luminosity functions for galaxies at $z=2.5$ (left) and $z=1$ (right) derived from a Monte Carlo simulation including galaxy merging $z$ associated starburst activity but no gas replenishment via accretion from the external environment. The dashed line is the original $(z=6)$ galaxy mass distribution (gaststars). The original gas supply is exhausted far too quickly, even by $z \sim 2$.

\subsubsection{ULIRG starbursts account for high-L tail}

The simulation shown in Fig. 8.31 includes gas accretion as formulated above but without the starburst activity associated with galaxy merging. Now, the ISM and stellar mass functions exhibit characteristics similar to those observed at $z=2.5$ and 1 , i.e., $\lesssim 50 \%$ gas mass and $10 \%$, respectively. However, even at $z=2.5$, the star formation luminosity function is lacking the power, even tail at the high-luminosity end. To reproduce the power-law tail, the starburst activity associated with galaxy merging is needed, as shown in Fig. 8.32. (The low-mass and low-luminosity ends of the distribution functions rise more steeply than is observed since no effort was made to model the behaviour there - this shallow slope is often attributed to starformation winds driving galactic mass-loss at velocities above the escape velocity of lower-mass galaxies.)

\subsection{Conclusions}

In these lectures, I have attempted to summarise a physically intuitive apIn these lecture characteristics of star formation in highredshift galaxies making use of our intuition gained from the low- $z$ Universe. mergers and accretion included but no SB
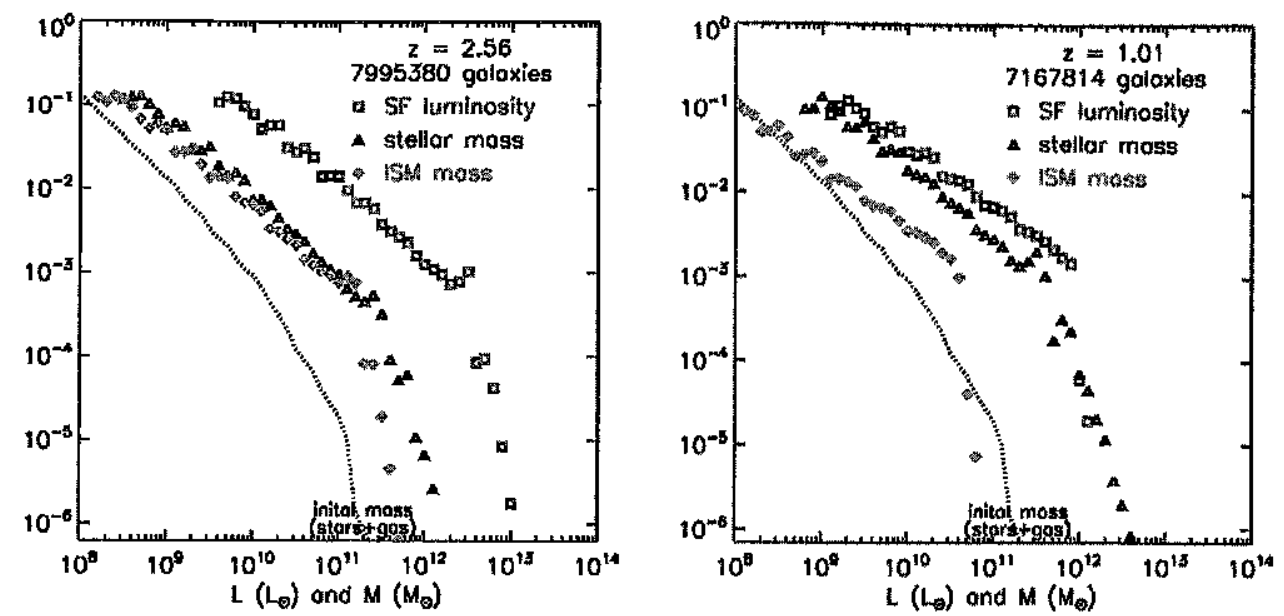

Fig. 8.31. Similar to Fig. 8.30 except that gas accretion at halo masses below $10^{12} M_{\odot}$ is included, following the prescription of (Dekel et al. 2009). In this simulation, the starburst activity associated with merging has been removed and the high end of the luminosity function naturally must follow the exponential form of the mass distribution.
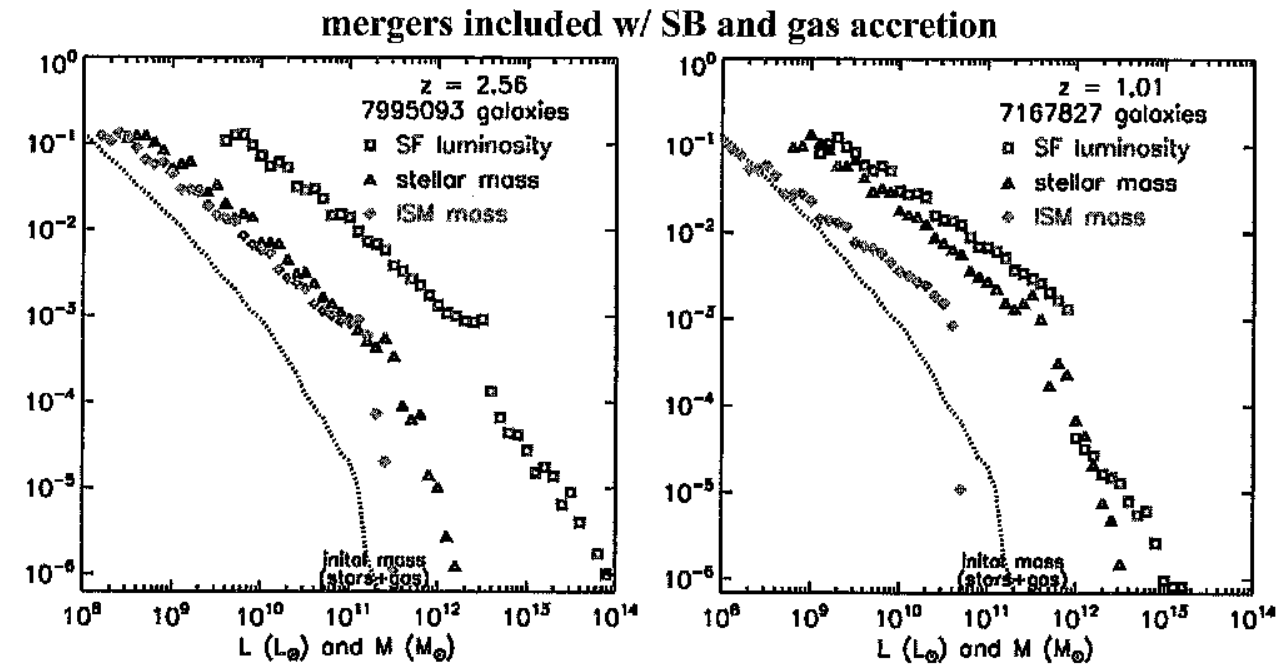

Fig. 8.32. Here both accretion and starburst activity during galaxy merging are included, giving a reasonable qualitative match to the observed high-end mass and luminosity functions.

I have devoted considerable effort towards developing an understanding of the molecular clouds (GMCs) in which most low-redshift star formation occurs in normal galaxies. 
I have also provided a summary of the physics of IR emission from optically thick dust clouds. Since there is very little systematic treatment from a theoretical point of view of the observed IR SEDs, I spent considerable effort to develop a systematic approach. Most of these IR sources are centrally heated by a starburst or AGN, so the theoretical framework for the dust heating and radiative transfer can be greatly simplified and distilled. Interpreting the observed SEDs is particularly prone to mistakenly evaluating a dust temperature from the wavelength of the SED peak when in reality a significant range of dust temperatures is contributing to the emergent flux and the peak is equally determined by the overall dust opacity (since obviously the radiation at a given wavelength cannot escape if the opacity is too high at that wavelength). I underscore also the point that the long-wavelength R-J tail fluxes should be used to derive dust (and ISM) masses - not total IR luminosities (as is commonly done for submm galaxies).

Given the known internal structure of the star-forming GMCs, it is reasonable to expect that star formation activity will have two modes: 1) a quiescent mode in which stars form in GMCs at a rate roughly proportional to the mass of $\mathrm{H}_{2}$ gas (accounting for the linear correlations observed between the $\mathrm{CO}$ emission and star formation tracers such as FIR over the disks of local galaxies including our own) and 2) a dynamically-triggered starburst mode in which the rate per unit gas mass is elevated by factors of $10-50$ accounting for the preferential formation of HIn regions in spiral arms, despite the presence of GMCs throughout the disk, and the high luminosity-to-gas ratios seen in local ULIRGs. For the starburst mode, collisions of GMCs may significantly increase the internal structure/density of the $\mathrm{H}_{2}$ gas and stimulated star formation due to supernovae and expanding HII regions.

At high redshift, the relative importance of these modes will be shifted, albeit in different directions, by two changes: 1) higher gas-mass fractions (increasing the quiescent mode) and 2) higher rates of galactic merging (increasing the starburst mode). Some observational determinations of the galaxy merger rates at high redshifts have shown very discrepant results for the evolution. However, my recent analysis of evolution in the frequency of galaxy close pairs from COSMOS data suggests merger evolution as $(1+z)^{2.3}$, in good agreement with the dark matter halo merger rates seen in simulations.

At high redshift the conditions in the gas may be quite different from those in the star-forming clouds at low redshift. It has been speculated that the stellar IMF might be top-heavy in starbursts or low-metallicity environments. This is not an insurmountable problem - observations of the $4.000 \AA$ break may be used to constrain the mass of low-mass stars. Until such observations are done, my conservative opinion would be to avoid the 'last refuge of scoundrels' since very little hard evidence is found in the local Universe to support such variations.

To test whether the ingredients discussed above provide a reasonable basis for understanding high-redshift galaxy evolution, I show the results of a Monte Carlo simulation starting at $z=6$ with 10 million haloes distributed in mass and with merging rates as found in $\Lambda$ CDM simulations. Starting with galaxies for which the gas content is $90 \%$ of the baryonic mass, the galaxies are evolved including both star formation modes and gas accretion. The simulations clearly show the need for gas replenishment through accretion from the external environment; otherwise, the $z=2$ and present-epoch gas contents are far too low.

This need for accretion is simply a reflection at high- $z$ of the well-known requirement of gas replenishment for the Milky Way. Specifically, for the Milky Way the SFR $\sim 3 M_{\odot}$ per year and the present gas content is $\sim 3 \times 10^{9} M_{\odot}$, implying an exhaustion timescale of only $1 \mathrm{Gyr}$. In the case of local galaxies, it does not appear that the accreting gas is HI since the high velocity $\mathrm{Hr}$ clouds do not constitute a sufficient influx. More likely the inflow is in the form of diffuse ionised hydrogen (HII) for which imaging with high sensitivity to low surface brightness emission is required. Clearly, this is an important direction for future observations.

Another conclusion from the simulations is that the starburst mode trig. gered by galactic merging is necessary to account for the high-luminosity power-law tail of the IR luminosity functions at high redshift. The quiescent mode cannot do this since the luminosity generated would simply reflect a scaled version of the galaxy mass function which is exponentially falling at the high mass end.

\section{Acknowledgments}

I would like to thank Zara Scoville for help in editing this manuscript and Andreas Schruba and Kevin Xu for a careful proofreading. I would also like to thank some of my close colleagues over the years who contributed much to this enjoyable research: Herve Aussel, Peter Capak, Peter Goldreich, Jeyhan Kartaltepe, Jin Koda, Colin Norman, Brant Robertson, Dave Sanders, Kartik Sheth, Phil Solomon and Min Yun.

\section{References}

Baan, W. A. Haschick, A. D. (1995), ApJ, 454, 745

Bahcall, J. N., Kirhakos, S., Saxe, D. H., Schneider, D. P. (1997), ApJ, 479, 642 Bigiel, F., Leroy, A., Walter, F. et al. (2008), AJ, 136, 2846 
Bigiel, F., Leroy, A. K., Walter, F. et al. (2011), ApJL, 730, 13 B. Tranx, M., Ford, H. (2007), ApJ, 670, 928 Bryant, P. M. Scoville, N. Z. (1999), AJ, 117, 2632

Bunt, W. B. Gordon, M. A. (1978), A B A, 63, 7

Burton, W. B. Gorer , Scoville, N. Z. et al. (2011), ApJ, 730, 68

Capak, P., Mobasher, B., Scoville, N. Z. et al. R. Yun, M. S. (2005), ApJ, 622, 346

Christopher, M. H., Scoville, N. Z., Sto (2010), MNRAS, 403, 274

Clements, D. L., Dunne, L., Eales, S. (2010), MNRAS, 403, 274 139

Clemens, D. P., Sanders, D. B., Scoville, N. Z. (1988), ApJ, 37, , 633, 857

Dale, D. A., Bendo, G. J., Engelbracht, C. W. et al. (2005), ApJ,

Dame, T. M., Hartmann, D., Thaddeus, P. (2001),

Dame, T. M., Thaddeus, P. (1985), ApJ, 297, 751 785

Dekel, A., Saric R. Astronomica Onghia,

$$
\begin{aligned}
& \text { Society Meet } \\
& \mathbf{4 3}, 417.06
\end{aligned}
$$

43, 417.06

Downes, D., Eckart, A. (2007), A\& A, 468, 57

Draine, B. T., Dale, D.A., Bendo, G. et al. (2007), ApJ, 663, 866

Draine, B. T., Li, A. (2001), ApJ, 551, 807

Draine, B. T, Li, A. (2007), ApJ, 657,810

Draine, B.

Drory, N., Alvarez, M. (2001), MNRAS, 327,697

Dunne, L., Eales, S. A. (2001, MESA, 533, A119

Eibaz, D., Dickinson, M., H., Pettini, M., Steidel, C. C., Reddy, N. A., Adelberger, Erb, D. K., Shapley, A. E., Pettin
K. L. (2006), ApJ, 644, 813

(2010), MNRAS, 401, 2245

(2006), Revista Mexicana de Astronomía y Astrofísica Conference Series, 26, 202

Gossive Stars in Interactive Binaries, N. St.-Louis \& A. F. J Moffat, 367, 689

A. $\mathrm{P}, \mathrm{K}$, J (1974), ApJ, 189, 441

Goldreich, P., Kwan, J. (1974, Soifer, B. T., Wilson, Graham, J. R., Carico, D. P., Math
T. D. (1990), ApJL, 354,5

T. D. (1990), ApJL, 354, 5

Heitmann, K., White, M., Wagner, C., Habib, S., H. (1971), ApJ, 163, 165

Hu, E. M., Cowie, L. L. (2006), Nature, 440, 1145

Ilbert, O., Salvato, M. Le Floc'h, E. et al. (2010), ApJ, 709, 644

(2010), ApJ, 714, 1746

Isella, A., Carpenter, H. W. W. (2005), MNRAS, 357, 565

H. W. W. (2005), MN

Jackson, J. M., Rathborne, J. M., Shah, R. 404

Jog, C. J., Ostriker, J. P. (1988), ApJ, 328, 404

Joseph, R. D., Wright, G. S. (1985), MNRAS, 214

Kennicutt, Jr., R. C. (1998), ARABA, 36, 189 . (2003), PASP, 115, 928

Kennicutt, Jr., R. C., Armus, L., Bendo, G. et al. (2JL ), P00, 132

Koda, J., Scoville, N., Sawada, T. et

Kroupa, P. (2001), (2012), ApJ, 745, 69

Leitherer, C., Schaerer, D., Goldader, J. D. et al. (1999), ApJS, 123, 3
MacKenty, J. W., Stockton, A. (1984), ApJ, 283, 64

Marchesini, D., van Dokkum, P. G., Förster Schreiber, N. M., Franx, M., Labbé, I., Wuyts, S. (2009), ApJ, 701, 1765

Matsunaga, K., Mizuno, N., Moriguchi, Y., Onishi, T., Mizuno, A., Fukui, Y. (2001), PASJ, 53, 1003

McLure, R. J., Cirasuolo, M., Dunlop, J. S., Foucaud, S., Almaini, O. (2009), MNRAS, 395, 2196

Murphy, E. J., Condon, J. J., Schinnerer, E. et al. (2011), ApJ, 737, 67

Murray, N., Quataert, E., Thompson, T. A. (2005), ApJ, 618, 569

Murray, N., Quataert, E., Thompson, T. A. (2010), ApJ, 709, 191

Norman, C., Scoville, N. (1988), ApJ, 332, 124

Ouchi, M., Shimasaku, K., Akiyama, M. et al. (2008), ApJS, 176, 3010

Peng, Y. J., Lilly, S. J., Kovač, K. et al. (2010), ApJ, 721, 193

Pollack, J. B., Hollenbach, D., Beckwith, S., Simonelli, D. P., Roush, T., Fong, W. (1994), ApJ, 421, 615

Radford, S. J. E., Delannoy, J., Downes, D. et al. (1991), in Dynamics of Galaxies and Their Molecular Cloud Distributions, IAU Symposium 146, F. Combes \& F. Casoli, eds., 303

Reddy, N. A. (2011), in UP2010: Have Observations Revealed a Variable Upper End of the Initial Mass Function?, M. Treyer, T. Wyder, J. Neill, M. Seibert, $\&$ J. Lee, eds., ASP Conf. Ser., 440, 385

Renzini, A., Buzzoni, A. (1986), in Spectral evolution of galaxies. Dordrecht: D. Reidel Publishing Co., 122, 195

Ripple, F., Heyer, M., Gutermuth, R., Snell, R. L. (2012), $M N R A S$, in preparation Robertson, B. E., Ellis, R. S., Dunlop, J. S., McLure, R. J., Stark, D. P. (2010), Nature, 468, 49

Robertson, B., Goldreich, P. (2012), ApJ, 750, 31

Rodighiero, G., Daddi, E., Baronchelli, I. et al. (2011), ApJL, 739, 40

Sanders, D. B., Mirabel, I. F. (1996), ARA\&A, 34, 749

Sanders, D. B., Scoville, N. Z., Soifer, B. T. (1991), ApJ, 370, 158

Sanders, D. B., Scoville, N. Z., Solomon, P. M. (1985), ApJ, 289, 373

Sanders, D. B., Soifer, B. T., Elias, J. H. et al. (1988), ApJ, 325, 74

Sakamoto, K., Aalto, S., Wilner, D. J., Black, J. H., Conway, J. E., Costagliola, F. et al. (2009), ApJL, 700, 104

Sakamoto, K., Scoville, N. Z., Yun, M. S., Crosas, M., Genzel, R., Tacconi, L. J. (1999), ApJ, 514, 68

Schruba, A., Leroy, A. K., Walter, F. et al. (2011), AJ, 142, 37

Scoville, N. (2003), Journal of Korean Astronomical Society, 36, 167

Scoville, N., Aussel, H., Brusa, M. et al. (2007), ApJS, 172, 1

Scoville, N., Aussel, H., Benson, A. et al. (2007), ApJS, 172, 150

Scoville, N., Capak, P., Salvatto, M. et al. (2012), ApJ, in preparation

Scoville, N. et al. (2012), ApJS, in preparation

Scoville, N. Z., Evans, A. S., Thompson, R. et al. (2000), AJ, 119, 991

Scoville, N. Z., Good, J. C. (1989), ApJ, 339, 149

Scoville, N. Z., Hersh, K. (1979), ApJ, 229, 578

Scoville, N. Z., Kwan, J. (1976), ApJ, 206, 718

Scoville, N., Norman, C. (1988), ApJ, 332, 163

Scoville, N., Norman, C. (1995), ApJ, 451, 510

Scoville, N. Z., Polletta, M., Ewald, S., Stolovy, S. R., Thompson, R., Rieke, M. (2001), $A J$, 122, 3017 
Scoville, N. Z., Sargent, A. I.; Sanders, D. B., Soifer, B. T. (1991), ApJL, 366, 5 Scoville, N. Z., Solomon, P. M. (1974), ApJL, 187, 67

Scoville, N. Z., Solomon, P. M. (1975), ApJL, 199, 105

Scoville, N. Z., Stolovy, S. R., Rieke, M., Christopher, M., Yusef-Zadeh, F. (2003), ApJ, 594, 294

Scoville, N., Young, J. S. (1983), ApJ, 265, 148

Scoville, N. Z., Yun, M. S., Bryant, P. M. (1997), ApJ, 484, 702

Scoville, N. Z., Yun, M. S., Sanders, D. B., Clemens, D. P., Waller, W. H. (1987), $A p J S, 63,821$

Shimasalku, K., Kashikawa, N., Doi, M. et al. (2006), PASJ, 58, 313

Shimasaku, K., Ouchi, M., Furusawa, H., Yoshida, M., Kashikawa, N., Okarnura S. (2005), PASJ, 57, 447

Solomon, P. M. Barrett, J. W. (1991), in Dynamics of Galaxies and Their Molecular Cloud Distributions, IAU Symp. 146, F. Combes \& F. Casoli, eds., p. 235

Tan, J. C. (2000), $A p J, \mathbf{5 3 6}, 173$

Tacconi, L. J., Genzel, R., Neri, R. Shapiro, K. et al. (2010), Nature, 463, 781

Tacconi, J. J. Genzel, R. Tecza, M., Gallimore, J. F., Downes, D., Scoville, N. Z (1999), ApJ, 524, 732

aert, E., Murray, N. (2005), ApJ, 630,167

van E. F. (2007), $A \& \& A, 468,627$

Veilleux, S., Kim, D. C., Sanders, D. B. (1999), ApJ, 522, 113

Young, J. S. Scoville, N. Z. (1991), ARABA, 29, 581

\section{Cosmological evolution of galaxies}

Isaac Shlosman

Department of Physics 83 Astronomy, University of Kentucky Lexington, $K Y$ 40506-0055, USA

shlosman@pa.uky.edu

\section{Abstract}

I review the subject of the cosmological evolution of galaxies, including different aspects of growth in disk galaxies, by focussing on the angular momentum problem, mergers, and their by-products. I discuss the alternative to merger-driven growth - cold accretion and related issues. In the followup, I review possible feedback mechanisms and their role in galaxy evolution. Special attention is paid to high-redshift galaxies and their properties. In the next step, I discuss a number of processes, gas- and stellar-dynamical, within the central kiloparsec of disk galaxies, and their effect on the larger spatial scales, as well as on the formation and fuelling of the seed black holes in galactic centres at high redshifts.

\subsection{Introduction: the paradigm of galaxy evolution}

The aim of these lectures is to review the main aspects of secular galaxy evolution, i.e., galaxy evolution on cosmological scales. Two issues stand out in this attempt. First, the subject is immense and cannot be covered within the scope of this chapter. And second, one cannot ignore the fact that galaxy evolution is part of the overall evolution in the Universe - from the largest spatial scales ruled by dark matter (DM) to the smallest ones taken over by dissipative baryons that can form stars and grow supermassive black holes (SMBHs). In other words, the process of galaxy formation can be influenced strongly by a huge range of spatial scales. Hence, our attempt to discuss secular galaxy evolution will be rather modest in depth, only highlighting those issues which appear to lie at the forefront of current research. Lastly, we shall focus on disk galaxy evolution and only briefly mention elliptical galaxies.

Galactic morphology is largely a reflection of underlying dynamical and secular processes on relevant scales. That morphological evolution does 